\begin{abstract}
Title of Thesis:

IN-FLIGHT ARRAY MEASUREMENTS OF TAIL ROTOR HARMONIC NOISE

D. Caleb Sargent, Master of Science, 2008

Thesis directed by: $\quad$ Professor Fredric H. Schmitz

Department of Aerospace Engineering
\end{abstract}

Acoustic data that utilized a unique flying microphone boom array was analyzed using a time domain averaging technique, based on a theoretical tail rotor one-per-rev signal, to obtain tail rotor acoustic time histories for forward and descending flight. This flying microphone array approach provides useful insight into the directivity and noise mechanisms associated with the periodic harmonic noise generated by the Bell 206B tail rotor. Comparisons between linear harmonic noise theory and the time averaged data show good agreement, indicating that the average harmonic noise of the $206 \mathrm{~B}$ is dominated by thickness noise near the tip-path-plane of the tail rotor and by loading noise at the out-of-plane microphone positions. The measured data also provides trends in the overall sound pressure level (OASPL) with respect to both forward velocity and descent angle. A significant deviation from the average SPL of the harmonic noise levels at high frequencies is also shown. 


\title{
IN-FLIGHT ARRAY MEASUREMENTS OF TAIL ROTOR HARMONIC NOISE
}

\author{
by \\ D. Caleb Sargent \\ Thesis submitted to the Faculty of the Graduate School of the \\ University of Maryland, College Park in partial fulfillment \\ Of the requirements for the degree of \\ Master of Science \\ 2008
}

Advisory Committee:

Professor Frederic H. Schmitz, Chair/Advisor

Professor James Baeder

Professor Christopher Cadou 
CCopyright by

D. Caleb Sargent

2008 


\section{Dedication}

To my grandfather, without whom, I might not ever have become interested in flight. 


\section{Acknowledgements}

I would like to thank my advisor Dr. Fred H. Schmitz for his advice and encouragement throughout this research. His support in both academic and life endeavors over the past two years has been invaluable in my learning and appreciation for the subject of rotorcraft acoustics. His continued enthusiasm and determination to continue learning after years of working in the industry are what drive me to continue in my current and future pursuits.

Similarly, I would also like to that the rest of the acoustics group at the University of Maryland working for Dr. Schmitz: Dr. Gaurav Gopalan, Dr. Sudarshan Koushik, Eric Greenwood and Rick Sickenberger for all of the invaluable help they have given me with this research and life here at the University of Maryland. I am not sure where I would be were it not for their help and patience.

Ben Sim also deserves many thanks for his help throughout this research. As a co-author on the American Helicopter Society Forum paper written, Ben was in large part responsible for the early work done for this research as he set out to show this novel averaging technique would even work. His input and criticism throughout the process were extremely helpful as was his hospitality and friendship during the flight testing process, a process that everyone who has been involved know can be quite trying.

I would like to thank all those that participated in the various flight tests. Though I did not participate in the 2006 test, I was involved with both the Summer 2007 and Spring 2008 tests and am well aware of the personal sacrifice required for such a test. Specifically I'd like to thank Eric Mouritsen, Sam Nowden and ARIS helicopter for their 
helicopter and facilities. I would also like to thank everyone else involved with the testing process.

Finally, I would like to thank my family and Kathryn for their help throughout this process. I would not be writing this today if it were not for their unyielding support. 


\section{Table of Contents}

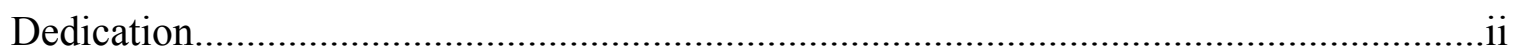

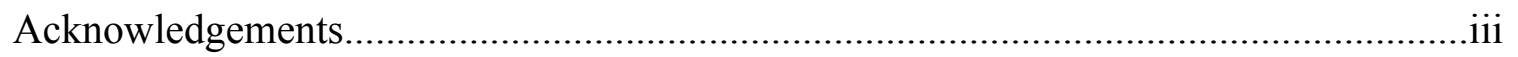

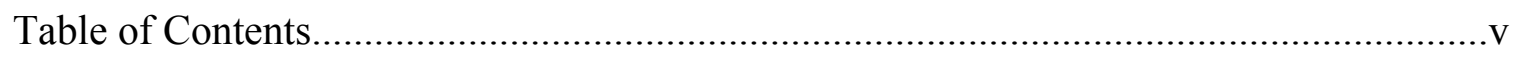

List of Tables....................................................................................................................

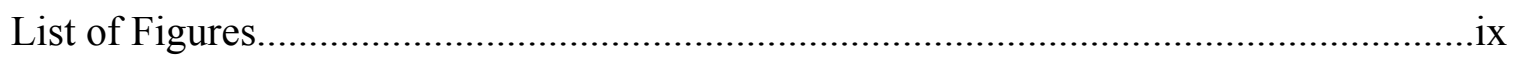

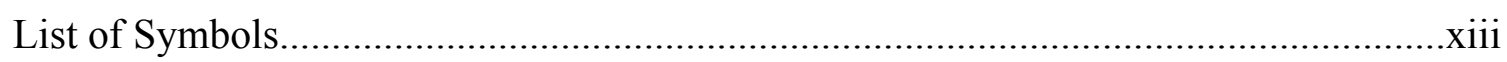

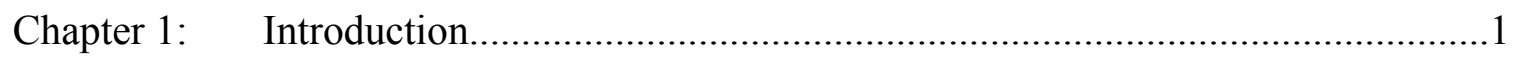

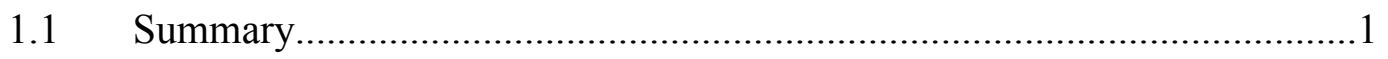

1.2 Tail Rotor Noise .............................................................................2

1.3 Some Previous Tail Rotor Noise Research............................................ 10

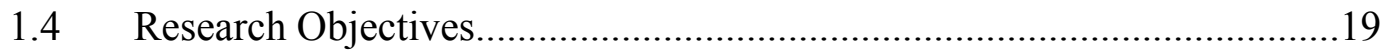

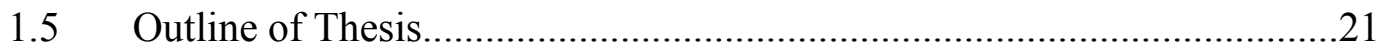

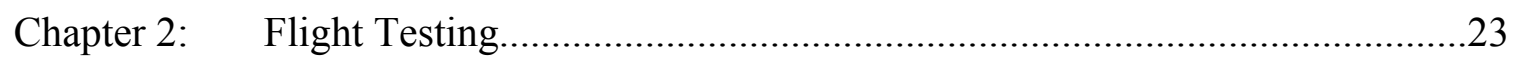

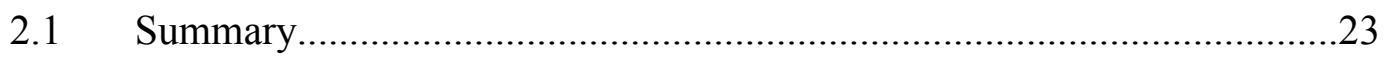

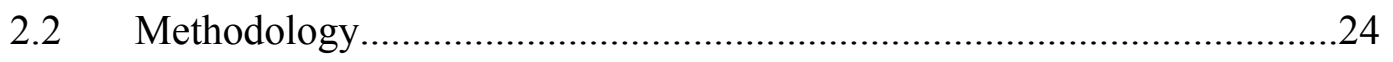

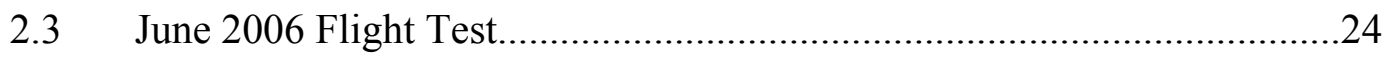

2.3.1 Air Data System...................................................................25

2.3.2 Main Rotor One-Per-Rev Sensor.............................................26

2.3.3 Pilot Display System..........................................................27

2.3.4 University of Maryland Boom Microphone Array......................28

2.3.5 Meteorological Conditions.................................................... 31 
2.3.6 Test Conditions.....................................................................32

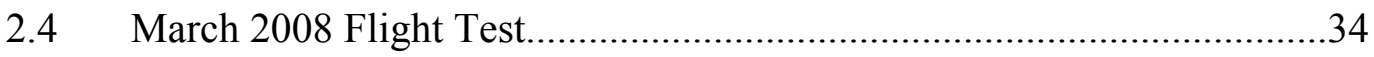

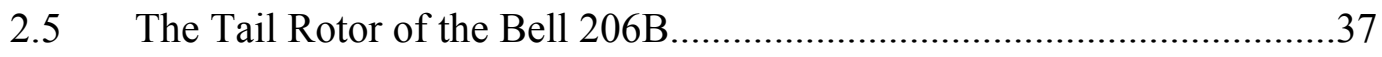

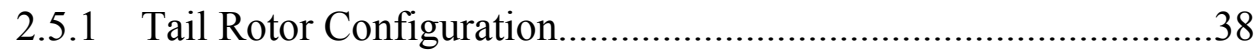

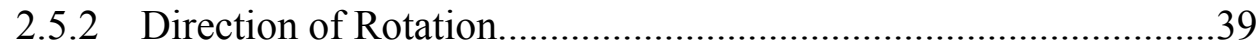

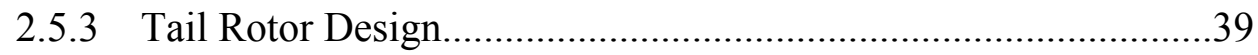

2.5.4 Geometry and Rotation Speed..............................................40

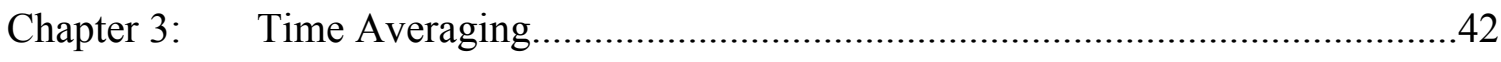

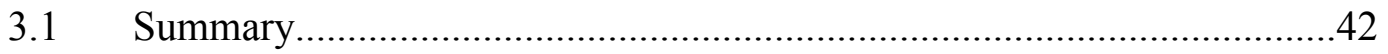

3.2 Main Rotor Time Averaging...............................................................42

3.3 Tail Rotor Time Averaging............................................................44

3.3.1 Drawbacks of Other Measurement Methods..............................45

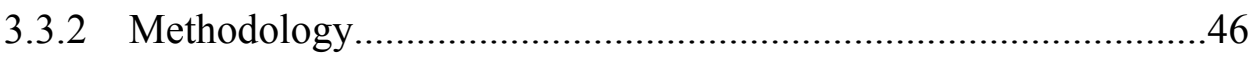

3.4 Discrete Fourier Transformation (DFT) Analysis..................................52

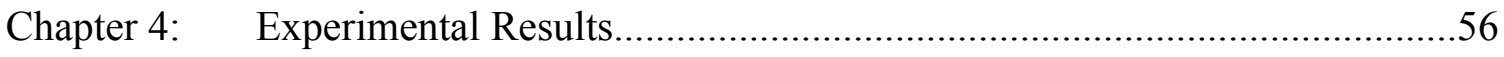

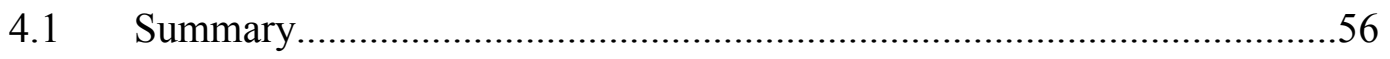

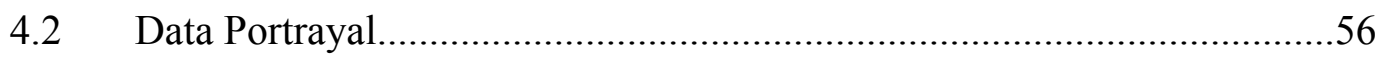

4.3 Level Forward Flight................................................................5

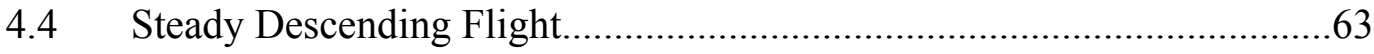

Chapter 5: $\quad$ Theoretical Tail Rotor Model......................................................... 70

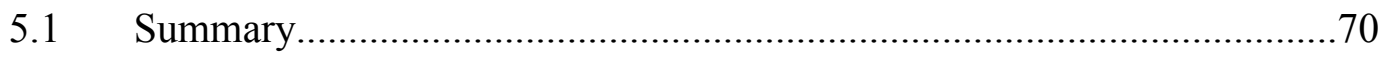

5.2 Rotor Harmonic Noise Prediction.................................................... 70

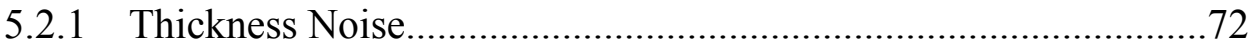

5.2.2 Loading Noise.................................................................. 74 


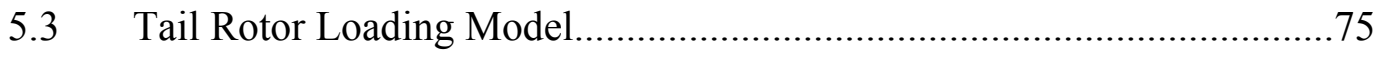

5.3.1 Calculation of Tail Rotor Thrust.....................................................76

5.3.2 Tail Rotor Trim........................................................................

5.3.3 Calculation of Tail Rotor Loading Distribution...............................80

5.4 Total Theoretical Noise .........................................................................83

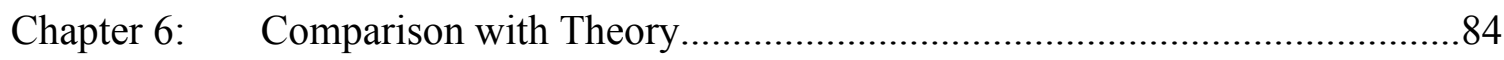

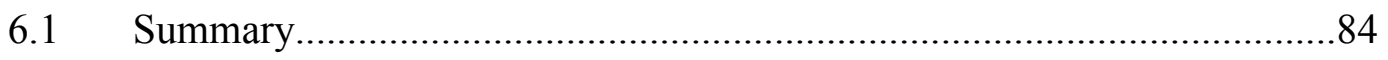

6.2 Steady Level Flight: Comparison with Theory.........................................84

6.3 Steady Descending Flight: Comparison with Theory.................................90

Chapter 7: Additional Considerations.....................................................................93

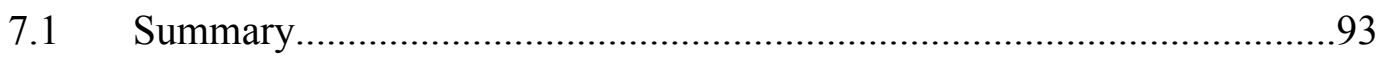

7.2 Standard Deviation within Spectral Averages............................................93

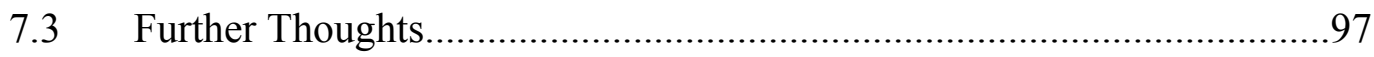

Chapter 8: Summary and Conclusions.....................................................................98

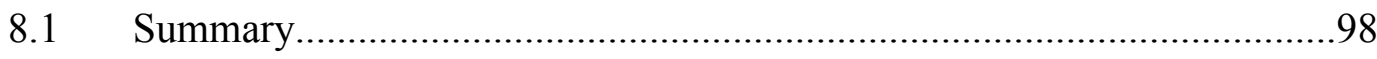

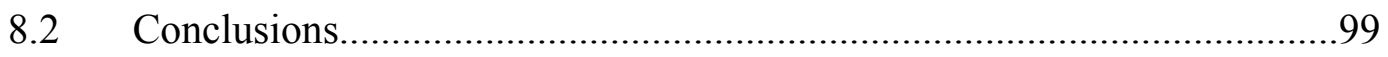

8.3 Recommendations for Future Work........................................................101

Appendix A: Bruel and Kjaer Microphone Data Sheet.................................................105

Appendix B: Tail Rotor Model Validation...............................................................107

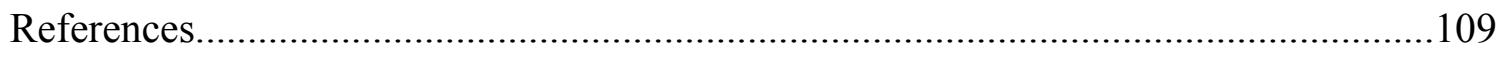




\section{List of Tables}

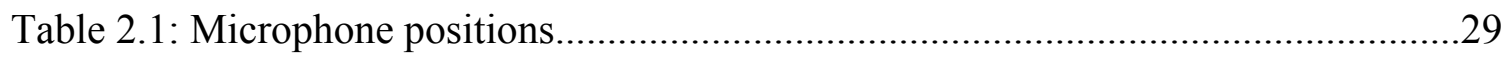

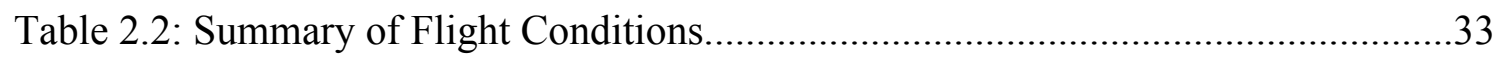

Table 2.3: Major parameters of the Bell 206B tail rotor..............................................41

Table 3.1: Standard deviation in main rotor rotational frequency.................................47

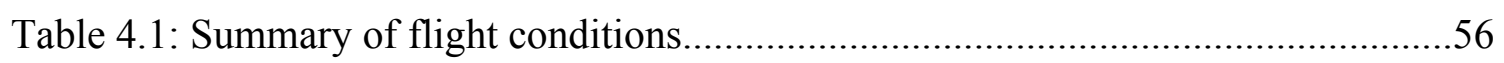

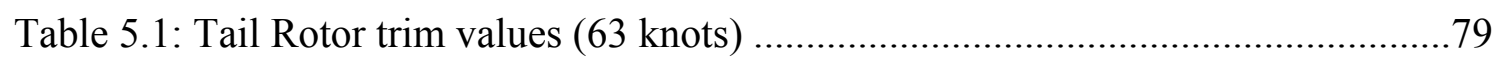




\section{List of Figures}

Figure 1.1: Dominant noise sources on a conventional helicopter

1

Figure 1.2: Sketch of the major source mechanisms of BVI noise 4

Figure 1.3: Sketch of a single main rotor vortex passing through the tail rotor TPP..... 5

Figure 1.4: Change in interaction azimuth angle for main rotor/tail rotor interaction........5

Figure 1.5: A sketch of a typical helicopter acoustic time history ..................................

Figure 1.6: Near in-plane acoustic power spectrum for 63 knot level flight.....................7

Figure 1.7: Atmospheric absorption of aircraft flyover noise versus frequency ................8

Figure 1.8: Filter characteristics for A-weighted sound level (dBA) .............................

Figure 1.9: A sketch of main rotor wake/tail rotor interaction in the plane of the TR......12

Figure 1.10: A simplified, planar interaction model of main rotor/tail rotor interaction...13

Figure 1.11: Difference in Full-scale Noise measurement techniques .19

Figure 2.1: Exterior dimensions of the Bell 206 B3 helicopter .23

Figure 2.2: 2006 flight test instrumentation .24

Figure 2.3: Space Age Control 100510 Swivel-Head air data system. .25

Figure 2.4: The PPDG display mounted on the Bell 206B. .27

Figure 2.5: Mic. locations on UMD Boom Microphone Array .29 
Figure 2.6: Coordinate system definition for Bell 206B tail rotor. .30

Figure 2.7: Sphere representing scaling used for acoustic measurements 31

Figure 2.8: Typical weather balloon data (June 14, 2006)

Figure 2.9: Flight testing procedure at Moffett Field, CA .33

Figure 2.10: Method for the determination of main rotor/tail rotor gear ratio. .36

Figure 2.11: Main rotor to tail rotor gear ratio statistical data. .37

Figure 2.12: Tail rotor configurations a) tractor b) pusher .38

Figure 2.13: The Bell 206B Tail Rotor .40

Figure 3.1: Main rotor time averaging for at -7.5 deg descent, $63.5 \mathrm{kts}$ .43

Figure 3.2: Generation of theoretical TR 1/rev signal from MR 1/rev signal .48

Figure 3.3: Acoustic data reduction technique for time averaging..... .51

Figure 3.4: TR time averaging schematic for Bell 206B at -7.5 deg descent, 63.5 kts.....52

Figure 3.5 : SPL spectra for A2 at $63.5 \mathrm{kts}$ a) $-7.5 \mathrm{deg}$ descent b) level flight. .55

Figure 4.1: Acoustic time average at microphone A1 as a function of forward velocity..58

Figure 4.2: Directivity pattern of tail rotor noise at 62 knots 61

Figure 4.3: Variation in OASPL as a function of airspeed for all microphones. 62

Figure 4.4: Variation of Microphone A1 with Descent Angle. .65 
Figure 4.5: Acoustic time average at microphone A3 as a function of descent angle.......68

Figure 4.6: Variation in OASPL as a function of descent angle for all microphones......69

Figure 5.1: Thickness noise directivity and pulse shape for Bell 206B tail rotor..... .73

Figure 5.2: Blade discretization for FWH implementation of thickness noise .74

Figure 5.3: Loading noise directivity and pulse shape for Bell 206B tail rotor..... .75

Figure 5.4: Flapping motion of tail rotor model (63 knots) .80

Figure 5.5: Out-of-Plane loading of the tail rotor model. .81

Figure 5.6 : In-plane loading of the tail rotor loading .82

Figure 5.7: Theoretical Combined Loading and Thickness Noise..... .83

Figure 6.1: Theory and Experiment (Steady Level Flight, 63 knots)

Figure 6.2: Sensitivity of Noise to Tail Rotor Thrust. .89

Figure 6.3: Theory and Experiment (63 kts, -7 deg descent) 91

Figure 6.4: Effective change in microphone position as descent angle differs .92

Figure 7.1: Spectral Average Measured on A4 $\pm 2 \sigma$. . .94

Figure 7.2: Standard Deviation of MR and TR Harmonic Amplitudes. 96

Figure A.1: Frequency Response of 4939 microphone (No Nose Cone) .105

Figure A.2: Frequency Response of 4191 microphone (No Nose Cone). 105 
Figure A.3: Mic nose cone and frequency response of $1 / 2$ in. microphone at 0 deg......106

Figure A.4: Free-field correction curve as a function of incidence angle.....................106

Figure B.1: Trim Validation (Collective) …........................................................107

Figure B.2: Trim Validation (Lateral Flapping) ..................................................108

Figure B.3: Trim Validation (Longitudinal Flapping)............................................108 


\section{List of Symbols}

A

$\mathrm{a}_{0}$

$\mathrm{C}_{\mathrm{do}}$

$\mathrm{C}_{\mathrm{la}}$

$\mathrm{C}_{\mathrm{P}}$

$\mathrm{C}_{\mathrm{T}}$

$\mathrm{C}_{\mathrm{W}}$

c

D

$\mathrm{dB}$

dBA

EPNL

f

$\mathrm{Hz}$

kts

$\mathrm{M}_{\mathrm{r}}$

$\mathrm{N}_{\mathrm{b}}$

OASPL

$\mathrm{Pa}$

$P_{n}$

$\mathrm{P}_{\mathrm{RMS}}$

$\mathrm{p}^{\prime}$

$\mathrm{R}$

RPM

$|\mathbf{r}|$ rotor disk area, $\mathrm{m}^{2}$

speed of sound $(342 \mathrm{~m} / \mathrm{sec})$

section zero-lift drag coefficient

section lift-curve slope, $[\mathrm{rad}]^{-1}$

main rotor power coefficient, $\mathrm{P} / \rho \mathrm{A}(\Omega \mathrm{R})^{3}$

thrust coefficient, $\mathrm{T} / \rho \mathrm{A}(\Omega \mathrm{R})^{2}$

weight coefficient, $\mathrm{W} / \mathrm{\rho A}(\Omega \mathrm{R})^{2}$

blade chord, $\mathrm{m}$

drag force, $\mathrm{N}$

decibels

A-weighted sound level, $\mathrm{dB}$

effective perceived noise level

equivalent flat plate area of fuselage, $\mathrm{m}^{2}$

hertz, 1 cycle/sec

nautical miles per hour

Mach number of acoustic source in radiation direction

number of blades

overall sound pressure level, $\mathrm{dB}$

Pascals, N/m

blade section lift pressure, $\mathrm{Pa}$

root mean squared pressure, $\mathrm{Pa}$

acoustic pressure, $\mathrm{Pa}$

rotor radius, $\mathrm{m}$

revolutions per minute

radiation vector magnitude, $\mathrm{m}$ 


\begin{tabular}{|c|c|}
\hline $\mathrm{r}$ & nondimensional radial distance \\
\hline $\mathrm{S}$ & blade surface, $\mathrm{m}^{2}$ \\
\hline SPL & sound pressure level, $\mathrm{dB}$ \\
\hline TPP & tip-path plane, deg \\
\hline $\mathrm{t}$ & time, sec \\
\hline $\mathrm{V}_{\infty}$ & free-stream velocity, $\mathrm{m} / \mathrm{sec}$ \\
\hline $\mathrm{V}_{\mathrm{n}}$ & velocity of fluid normal to the blade surface, $\mathrm{m} / \mathrm{sec}$ \\
\hline$\alpha_{\mathrm{TPP}}$ & rotor tip-path plane angle of attack, deg \\
\hline$\beta_{1 \mathrm{C}}$ & longitudinal flapping angle of tail rotor, deg \\
\hline$\beta_{1 \mathrm{~S}}$ & lateral flapping angle, deg \\
\hline$\delta_{3}$ & pitch-flap coupling angle, deg \\
\hline$\theta_{0}$ & tail rotor collective pitch, deg \\
\hline$\kappa$ & induced power factor \\
\hline$\lambda$ & rotor inflow ratio \\
\hline$\mu$ & rotor advance ratio \\
\hline$\rho_{0}$ & air density, $\mathrm{kg} / \mathrm{m}^{3}$ \\
\hline$\sigma$ & standard deviation \\
\hline$\sigma$ & rotor solidity \\
\hline$\tau$ & retarded time, sec \\
\hline$\psi$ & rotor azimuth angle, deg \\
\hline$\Omega_{\mathrm{MR}}$ & rotational speed of main rotor, $\sim 6.5 \mathrm{~Hz}$ for Bell $206 \mathrm{~B}$ \\
\hline$\Omega_{\mathrm{TR}}$ & rotational speed of tail rotor, $\sim 41.5 \mathrm{~Hz}$ for Bell $206 \mathrm{~B}$ \\
\hline$\square^{2}$ & wave operator \\
\hline
\end{tabular}




\section{Chapter 1 Introduction}

\subsection{Summary}

This first chapter provides a background of previous research and thought that has gone into understanding and reducing the tail rotor noise problem. After an introduction to the major mechanisms associated with rotors, with an emphasis on those mechanisms that are most important to the tail rotor, the significance of the tail rotor as an acoustic source is discussed. A survey of the most important work pertaining to the tail rotor problem is then presented. Finally, the major objectives of this research and the order in which they will be presented are discussed.

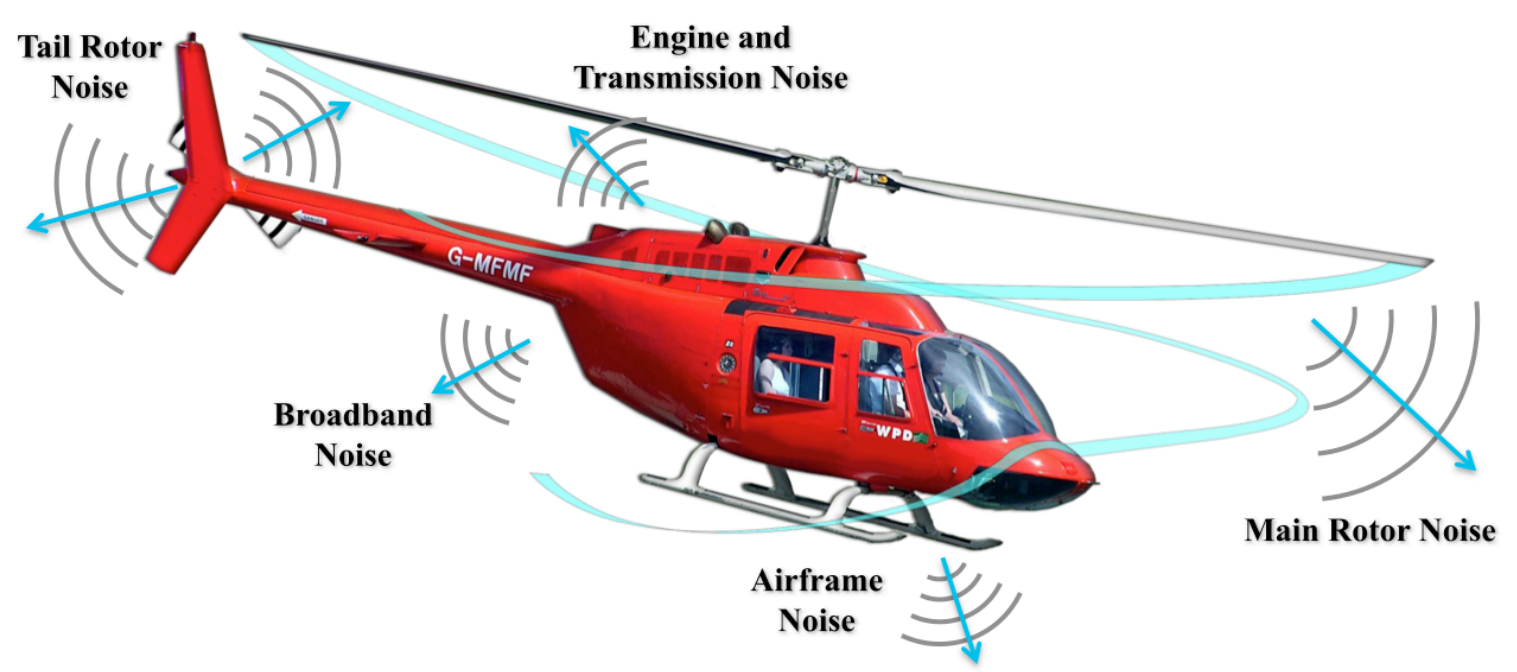

Figure 1.1: Dominant noise sources on a conventional helicopter 


\subsection{Tail Rotor Noise}

As anyone who has been near a helicopter surely knows, helicopters have a tendency to be very noisy aircraft. This noise, which can emanate from any number of sources on a helicopter, including the main rotor, engine, fuselage, transmission, and tail rotor (Figure 1.1), is at least partly responsible for the minimal use of helicopters as a means of civilian transport when compared to fixed wing aircraft. The focus of this thesis is the noise that is produced by the tail rotor, specifically the harmonic noise produced by the tail rotor in both forward and descending flight.

In recent years, tail rotor acoustics has become the focus of an increasing amount of research. This increase in attention has not only been due to the demands for a "quiet" helicopter from both civil and military consumers, but also the growing understanding that the tail rotor is one of the primary sources of noise for many helicopters operating in many flight conditions - this is especially true for light and medium weight helicopters.

The tail rotor is in many ways similar to the main rotor, and as a result, it is subject to many of the same noise mechanisms that are associated with the much larger main rotor. In order to understand the importance of the tail rotor, these harmonic noise mechanisms must be understood. There are four major sources of harmonic rotor noise.

- Thickness Noise: Thickness noise is a direct result of the displacement of the fluid in which the rotor is operating (in this case, air) by the blade. Present in all operating conditions, thickness noise is modeled mathematically as a monopole source $^{31}$ with a characteristic large negative pulse shape and is loudest in the plane of the rotor. 
- Loading Noise: This noise is due to the steady and unsteady aerodynamic forces acting on the medium and is highly dependent on the flight condition of the rotor, whether it is the main or tail rotor. Loading noise is generally modeled mathematically as a distribution of dipole sources, with the lift component radiating predominantly above and below the plane of the rotor in question, and the generally smaller drag component radiating closer to the plane of the rotor.

- High Speed Impulsive (HSI) Noise: Though generally of less concern for tail rotors, HSI noise can be considered to be an extreme case of thickness noise (for very high advancing tip Mach numbers) and is characterized by a very intense pulse that is strongly influenced by the transonic effects on or near the blade surface. These transonic effects can result in the formation of a shock on the blade surface, which can extend past the tip of the blade and into the far-field (also known as "delocalization") ${ }^{4,5}$. However, tail rotors are often designed to operate at tip Mach numbers and aspect ratios low enough to avoid HSI ${ }^{6,7,8}$ and other compressibility related effects. Though this results in a slight degradation in performance, the losses are less than might be observed when reducing the tip tip Mach number of the main rotor.

- Blade-Vortex Interaction (BVI) Noise: This noise is perhaps the most heavily investigated main rotor noise phenomenon, as it is characterized by the typical "Wop Wop" noise heard in the far-field, in front of many helicopters. When it occurs, this noise tends to become the loudest and most objectionable noise source on the helicopter. BVI noise is a result of the interaction between the tip vortices shed by preceding blades and the following blades of the same rotor 
system, as sketched below in Figure 1.2. The BVI pattern is periodic for any equally spaced multi-bladed rotor system.

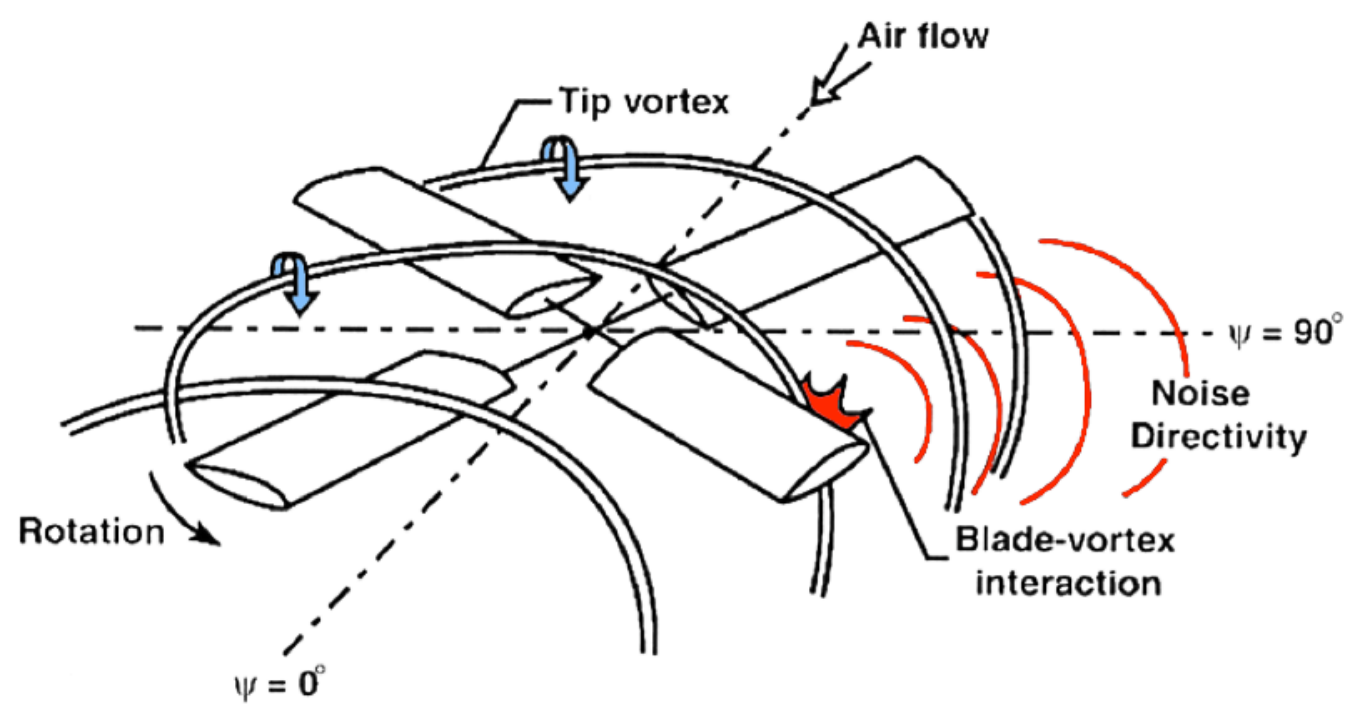

Figure 1.2: Sketch of the major source mechanisms of BVI noise

Though BVI is generally of greater concern for the main rotor, it can also occur on the tail rotor. It is most likely to occur at high tail rotor thrusts and low inflow conditions.

Another type of BVI noise can also occur on tail rotors where the tip vortices shed by the main rotor passes through, or in close proximity to the tail rotor blades. This operational situation is sketched below in Figure 1.3.

In this case, a vortex passes through the operational plane of the tail rotor at angles close to normal to the tail rotor tip path plane. In general, the tail rotor azimuth position at the time of interaction is dependent upon the gear ratio between the main and tail rotors. For integer gear ratios between the main and tail rotor tranmissions, the noise of the main and tail rotors have a common period and the 


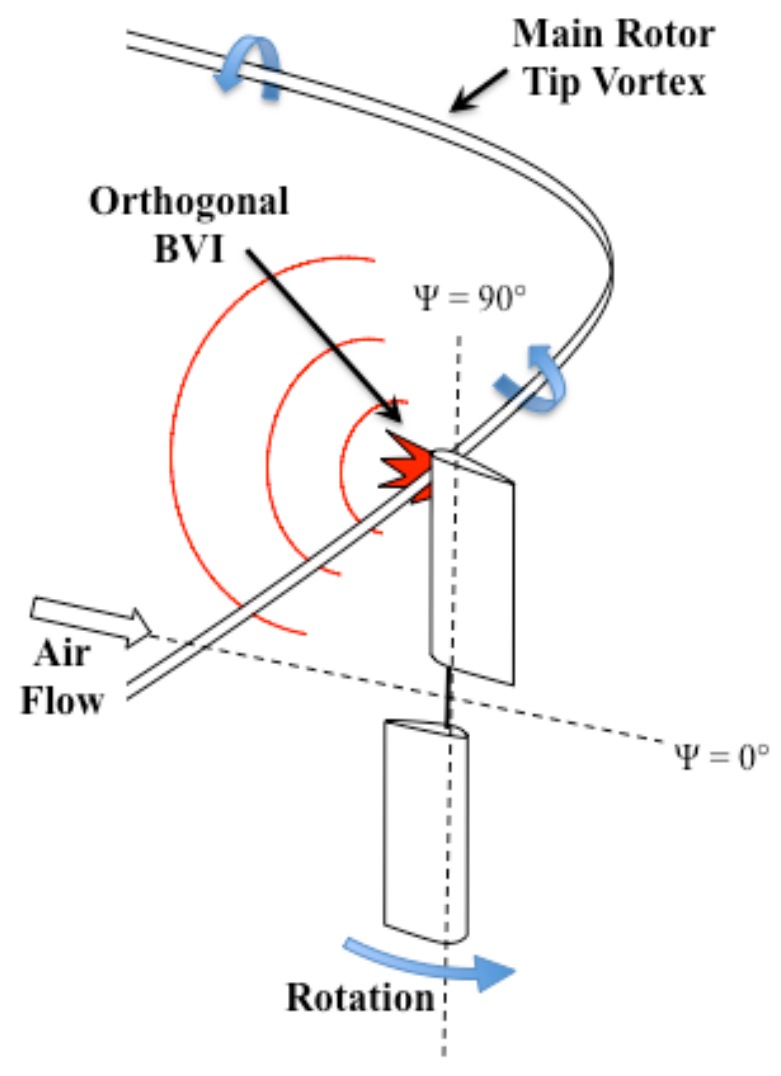

Figure 1.3: Sketch of a single main rotor vortex passing through the tail rotor TPP

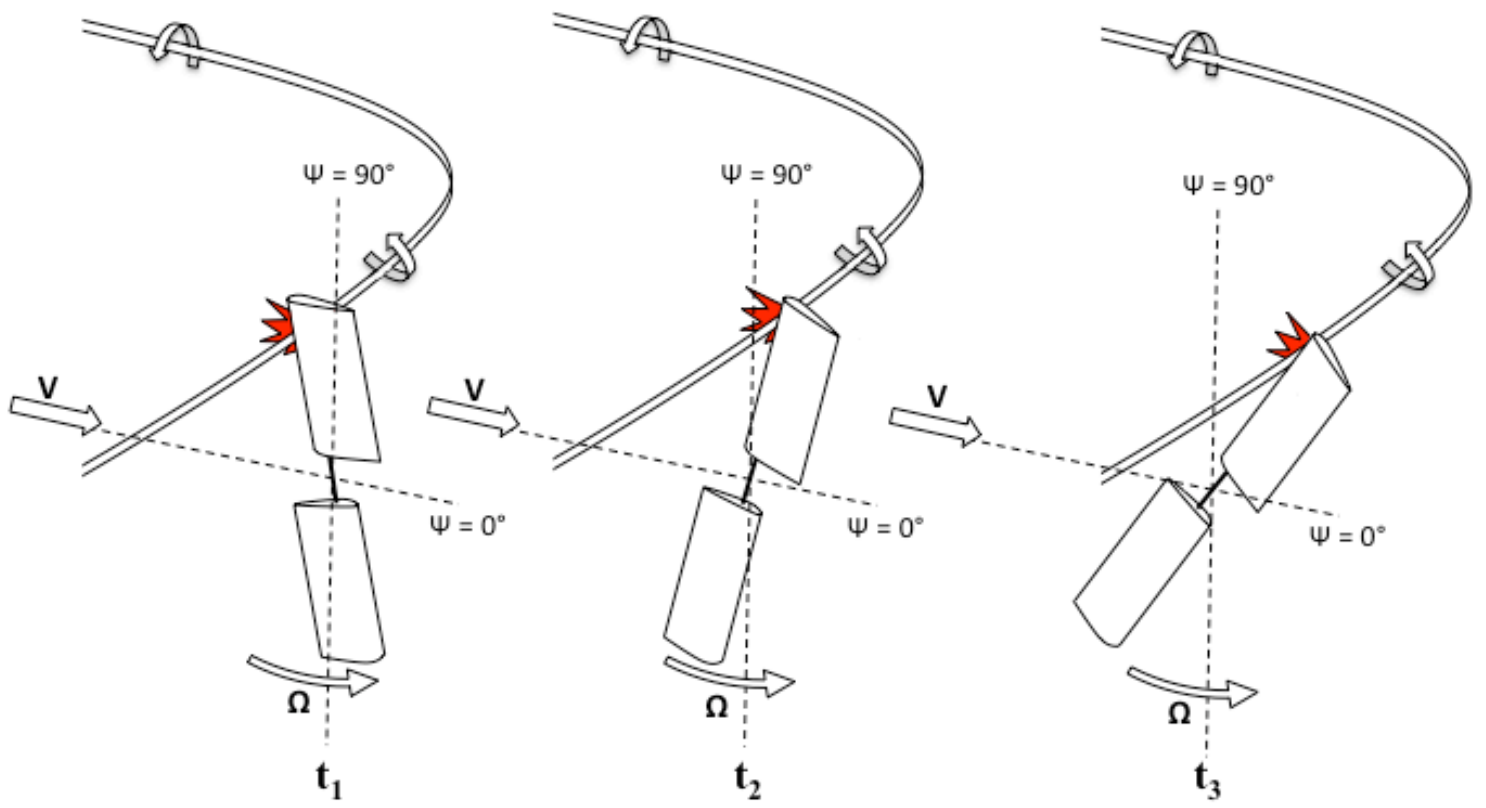

Figure 1.4: Change in interaction azimuth angle for main rotor/tail rotor interaction 
noise consists of multiple harmonics of the main rotor. If however, as is usually the case, the gear ratio between the main and tail rotor is chosen to be a non-integer multiple of the main rotor, then the noise is made up of two harmonic series - one for each rotor period. More importantly, the azimuth location where the tail rotor blade "cuts" the main rotor tip vortices changes with time (See Figure 1.4). This changing azimuth position results in a non-stationary source of noise.

The importance of the tail rotor stems in large part from the fact that tail rotors operate at nearly the same tip Mach number as main rotors, though at a significantly higher rotational rate. This higher rotational rate results in higher frequency noise. Figure 1.5 shows a typical sketch of an acoustic time history for a Bell 206B helicopter over the span of a single, two-bladed main rotor revolution. The low frequency nature of the main rotor harmonic noise is indicated by the red dotted line, with a significantly higher frequency main rotor blade-vortex interaction pulse occurring at the apex of each low frequency pulse. Overlaid on top of this main rotor noise is the higher frequency tail rotor noise, indicated by the largely negative peaks occurring at a rate approximately 6.5 (6.472) times the main rotor revolution rate. The frequency at which this tail rotor noise occurs is a function of the main rotor to tail rotor transmission gear ratio, which is discussed further in Section 2.3.

The different frequency content of each source can be seen in Figure 1.6. Main rotor harmonic noise clearly dominates the lower end of the frequency spectrum. However, in the mid frequency range, both tail rotor harmonic noise and BVI and HSI noise dominate, the importance of each with respect to the other being highly dependent on flight condition. 


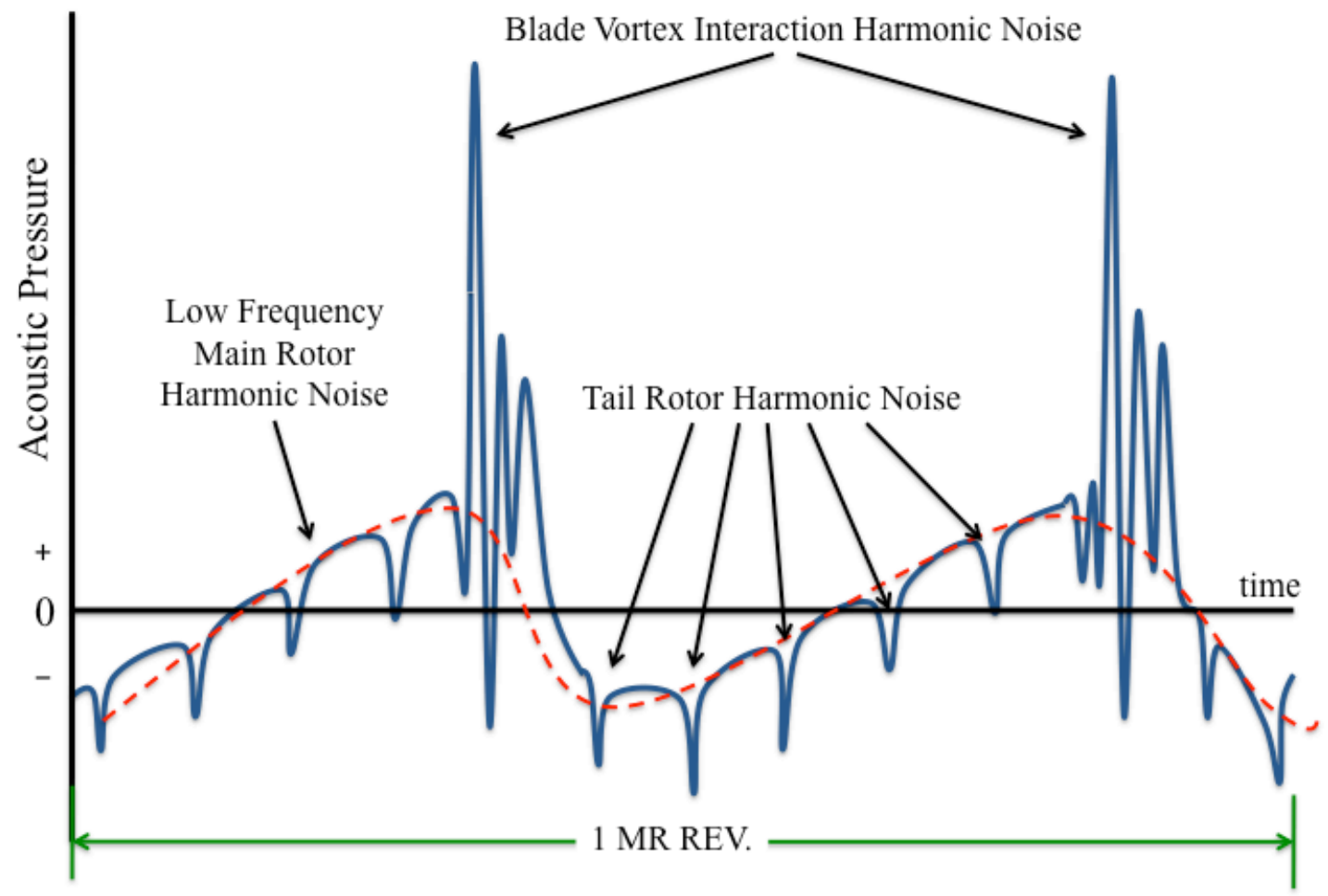

Figure 1.5: A sketch of a typical helicopter acoustic time history

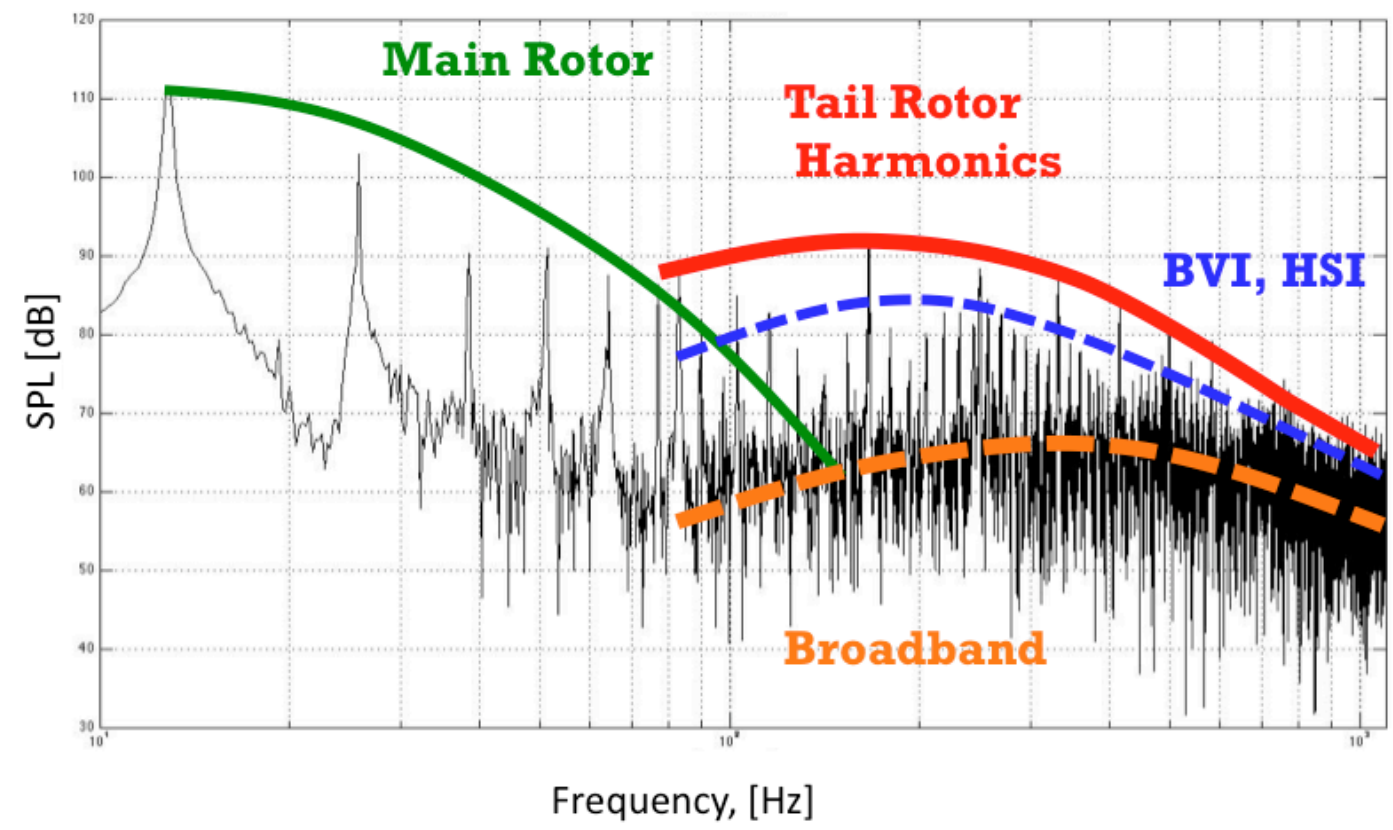

Figure 1.6: Near in-plane acoustic power spectrum for 63 knot level flight 
Higher frequency noise can also be generated by the transmission, engine, and various other broadband sources. However, these higher frequency noise sources are also efficiently reduced in amplitude by atmospheric absorption, making higher frequency noise less important to the far-field observer.

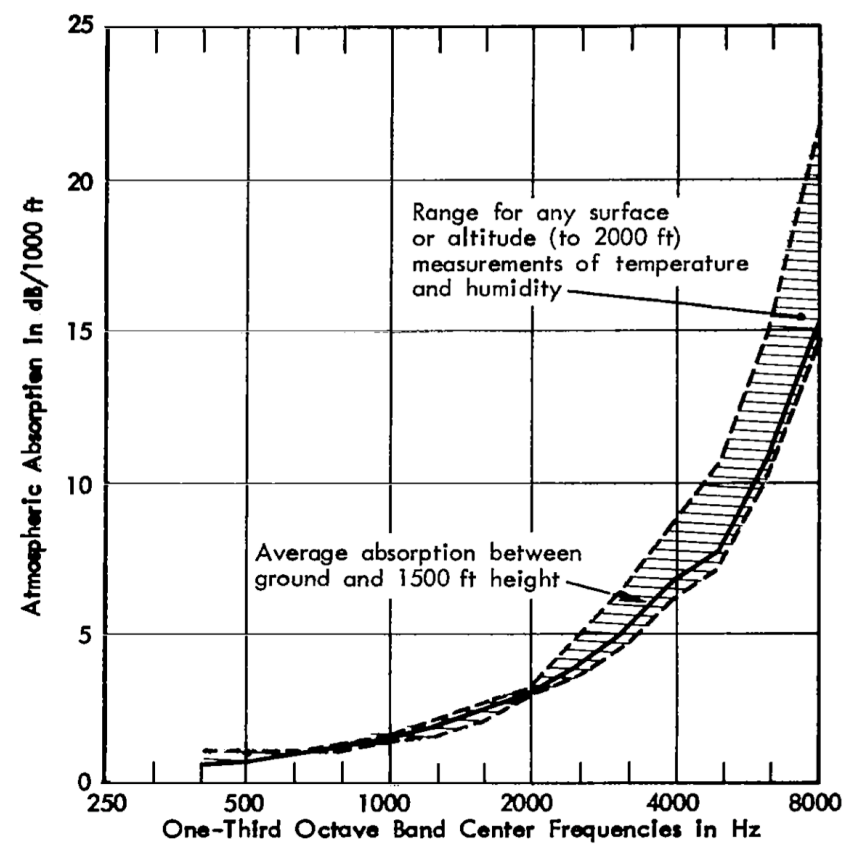

Figure 1.7: Atmospheric absorption of aircraft flyover noise versus frequency ${ }^{3}$

It has been well demonstrated that the atmosphere plays an important role in the long distance propagation of noise. Figure 1.7 shows that the level of atmospheric absorption is heavily dependent on the frequency content of the noise. This figure, which was obtained during a NASA test to study the effect of the atmosphere on aircraft flyover noise ${ }^{3}$, indicates that noise at frequencies above a few thousand Hertz is attenuated drastically ${ }^{1}$. This means that most of the noise generated at frequencies higher than the tail rotor and main rotor BVI and HSI is heavily absorbed by the atmosphere and is often not heard at all until the helicopter is in very close proximity to the observer. 
Lower frequency noise, which is for the most part associated with the main rotor, is of lesser importance for an entirely different reason. Clearly this low frequency noise travels over very long distances with very little absorption by the atmosphere. Noise in this frequency range is however barely audible to the human ear and much less annoying to a human observer. Perhaps the most common measures of annoyance and loudness, the effective perceived noise level (EPNL) and A-weighted sound level (dBA) have been shown to have very good correlation with human testing ${ }^{1}$. Figure 1.8 , shows the filter characteristics for the A-weighted sound level and demonstrates that a noise level near 20 $\mathrm{Hz}$ would have to be at least $50 \mathrm{~dB}$ greater in intensity than a $1,000 \mathrm{~Hz}$ noise to be important. As shown in Figure 1.6, noise below the tail rotor range, that is, noise below $80 \mathrm{~Hz}$, would have very little effect on the level of annoyance that is experienced by an observer $^{2}$.

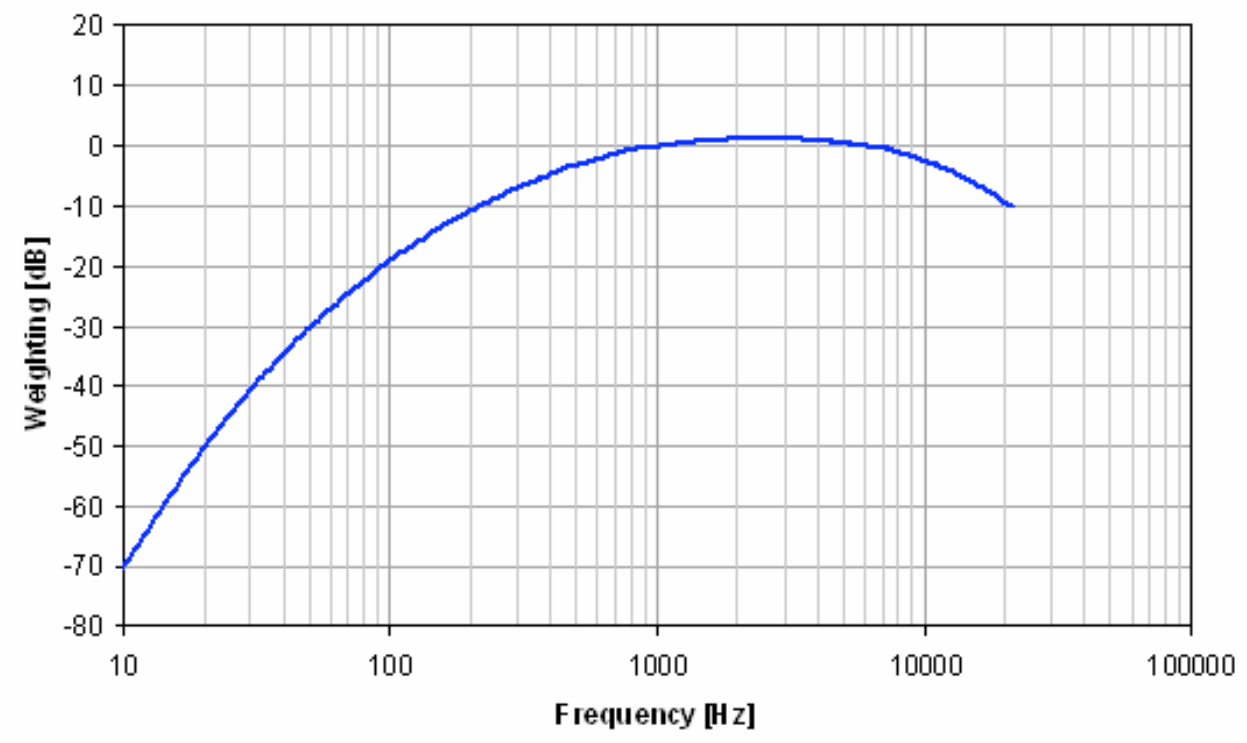

Figure 1.8: Filter characteristics for A-weighted sound level (dBA)

The combination of the human ear's sensitivity to certain frequencies and the effect of atmospheric absorption place the frequency range associated with tail rotors (as well as 
main rotor blade-vortex interaction (BVI) and high speed impulsive noise (HSI), which when they exist, can supersede the tail rotor in importance) at the forefront of the rotorcraft noise problem.

\subsection{Some Previous Tail Rotor Noise Research}

In the late 1960's, the helicopter industry first recognized the importance of tail rotor noise as a problem. Lynn et al, at Bell Helicopters concluded that, "for nearly all flight conditions, the tail rotor is the predominant noise source for single rotor helicopters."6 Though this may have been somewhat of an overstatement, it was certainly realized by the industry as a whole that the tail rotor was a significant contributor to the overall noise generated by a helicopter.

An engineering investigation of the tail rotor as an important noise source occurred under the "Quiet Helicopter Program" that began its first phase in 1969 and completed a second phase in the early 70 's. ${ }^{9,10}$ The first phase of this program focused primarily on what was assumed to be the major noise producer on the OH-6A helicopter - the tail rotor. The effects of changing blade number, camber and blade phase angle were investigated. However, the noise reductions associated with these parameter changes were not substantial enough and caused other performance penalties. They did however find that lowering the tail rotor tip speed alone could make substantial reductions in tail rotor noise, with only a very small reduction in performance.

At around the same time, Westland Helicopters Ltd. Began a tail rotor noise research program because of a unique "burble" sound emanating from the Westland Lynx helicopter. Under the leadership of John Leverton, tail rotor noise was also found to be a 
very important contributor to the noise levels generated by helicopters, both at distance and during overhead flight. ${ }^{8,11}$ Perhaps more significantly, Leverton concluded that it was not only many of the mechanisms attributed to isolated rotor noise that were important to the tail rotor problem, but also the noise created as a result of the interaction between the tail rotor and main rotor wake.

The studies conducted by Westland identified that a characteristic "burble" noise was associated with the main rotor wake/tail rotor interaction on the Westland Lynx helicopter and they deemed that this interaction was the most significant source of tail rotor harmonic noise for the Lynx helicopter. As illustrated previously (Figure 1.3), this interaction occurred when the tip vortices shed by the rear of the main rotor were being "chopped" by the blades of the tail rotor. A simplified schematic of this interaction in the plane of the tail rotor is shown in Figure 1.9. Leverton also identified a weaker "overhead interaction" noise that was radiated downwards and was a result of the interaction between the tip vortex of the leading edge of the rotor disk and the tail rotor. This interaction was thought to be of less importance as this main rotor tip vortex is heavily impacted by the main rotor hub and fuselage, whereas the vortices from the rear of the rotor can travel to the tail rotor on a relatively unimpeded path. However, it was clear that the relative importance of one noise or the other was a function of the location of the tail rotor with respect to the main rotor wake. This location changes as a function of both the helicopters structural layout as well as the flight condition and strength of the main rotor vortices.

From a simple theoretical analysis, Leverton et al concluded that the noise generated by this interaction occurred at frequencies of $n 4 \Omega_{\mathrm{TR}} \pm \mathrm{m} 4 \Omega_{\mathrm{MR}}$ where $\mathrm{n}$ and $\mathrm{m}$ are integer 
values for the four bladed main and tail rotors of the Lynx helicopter. As discussed previously, in helicopters with a non-integer gear ratio between the main and tail rotors, this event is aperiodic with respect to both the main rotor and tail rotor rotation period. Therefore, this acoustic event would not appear in a typical time average, a process which by its very nature rids itself of any aperiodic noise. This is not to say that when strong enough, the phenomenon would not show up in a time domain analysis. However, in any given rotor revolution, the event would appear at a different time (and thus azimuth location). The initial study conducted by Westland focused on a tail rotor that was of the advancing side up (ASU) configuration (CCW in Figure 1.9). They soon found that by changing the direction of rotation, this burble noise was quickly superseded in importance by the classical thickness and loading noise produced by the tail rotor, and in some cases, it disappeared completely.

Examining the differences between an ASU tail rotor configuration and the ASD configuration is perhaps the best way of explaining why the rotation direction plays such a large role in the importance of the interaction noise. The top rotor configuration in Figure 1.10 shows a simplified sketch of the interaction model for an ASU configuration.

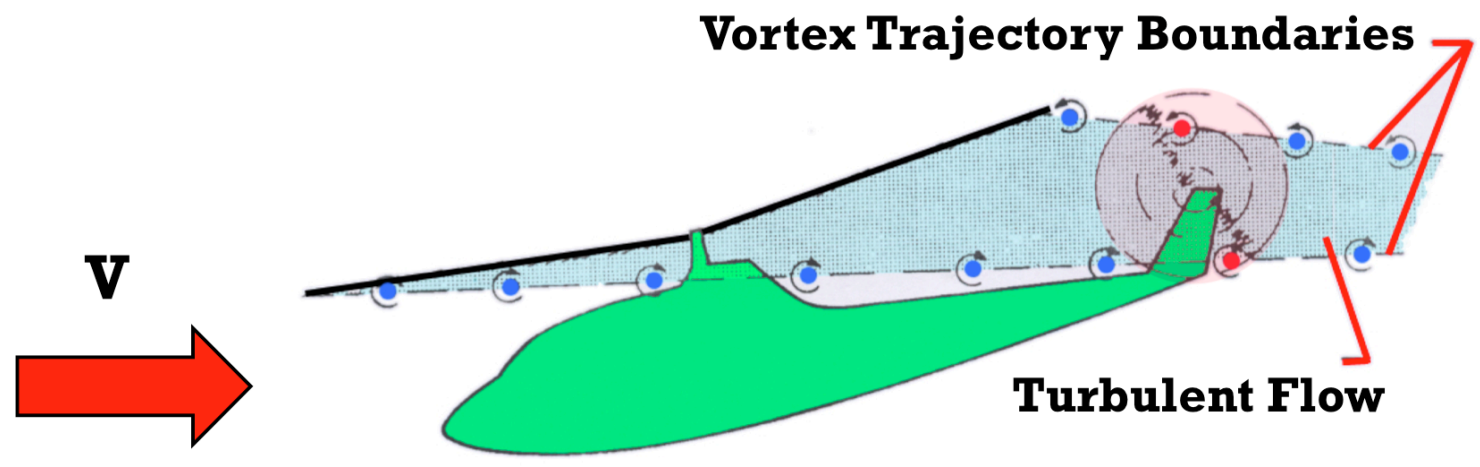

Figure 1.9: A sketch of main rotor wake/tail rotor interaction in the plane of the tail rotor 


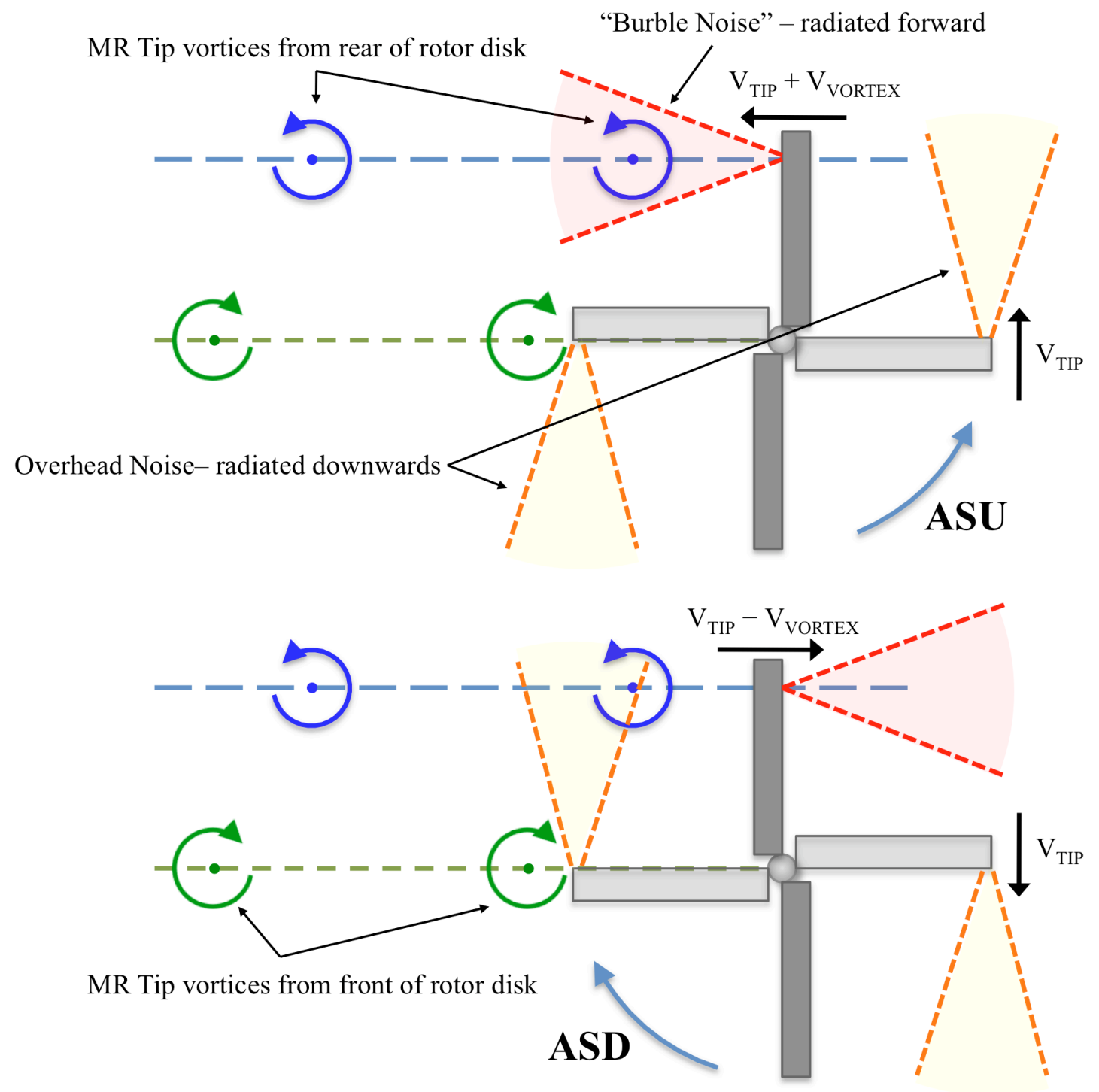

Figure 1.10: A simplified, planar interaction model of main rotor/tail rotor interaction

The main rotor tip vortices, indicated in blue, from the rear of the rotor disk interact with the tail rotor on the advancing side of the rotor disk, where the tip Mach numbers are greatest and the disturbances caused by the main rotor/tail rotor interaction are radiated most efficiently. This interaction results in what Leverton referred to as "Burble noise", a loud, impulsive noise radiated forward of the helicopter. In the ASD configuration, the tip Mach number at which this interaction occurs is much smaller, causing much less noise 
to be radiated towards the rear of the helicopter. In addition to the "Burble noise", Figure 1.10 also indicates the "overhead interaction" noise mentioned by Leverton. This interaction occurs at an intermediate tip Mach number and this vortex will likely be heavily influenced by the main rotor downwash, hub, and fuselage. This combination of factors results in a noise that is directed both upward and downward of the rotor but that is not as intense as the noise that is radiated by disturbances that pass through the advancing side of the tail rotor. It is also clear from Figure 1.10 that the location of the vortices plays an extremely important role in the relative importance of any particular noise, however, given the configuration of most modern tail rotors, the ASD rotor would appear to be preferable.

The conclusions to this study, which are perhaps best summarized in reference 8 , found that while the noise associated with the interaction between the main rotor wake and tail rotor wake was capable of being dominant, careful design of both rotors, especially the use of an ASD tail rotor, can greatly reduce this noise.

Subsequent studies by Pegg and Shidler at NASA Langley confirmed that, when main rotor wake/tail rotor interaction occurred (they used an ASU rotor and varied the tail rotor location and trim), it was the dominant source of tail rotor noise. ${ }^{12}$ They also found that this noise occurred at similar frequencies to those found by the Westland investigation. This research involved a variable geometry $1 / 16^{\text {th }}$ scale model of a UH-1 series helicopter with a tail rotor that could be operated in both directions. This study used a simple modified momentum theory analysis to predict the location of the main rotor wake with respect to the tail rotor. 
In 1985/1986 George and Chou performed a fully theoretical study of the main rotor wake/tail rotor interaction for the UH-1D and also concluded that the main rotor wake was the strongest contributor to the harmonic tail rotor noise. ${ }^{13}$ This theoretical study modeled the interaction as a concentrated vortex traveling across the tail rotor disk, with the acoustic pressure time history being computed using Amiet's two-dimensional aerodynamic theory. Similar to previous work, the results indicated that the noise was highly dependent on the location of the tail rotor with respect to the main rotor wake. Tadghighi took a similar approach a few years later in 1988, with the same result. ${ }^{17}$

At this point in history, it was generally assumed by the helicopter acoustic community that the major cause of tail rotor noise was due to the main rotor wake/tail rotor interaction. However, in 1988/1989, work began to emerge that indicated quite the opposite.

Fitzgerald and Kohlhepp ${ }^{14}$, as well as Martin et al ${ }^{15}$, conducted Mach scale 1/5.727 tests of an unnamed operational Sikorsky helicopter in the NASA Langley $14 \mathrm{ft}$ x $22 \mathrm{ft}$ wind tunnel that showed that the tail rotor actually produced more noise when in isolation, than when operated in the presence of a main rotor. They attributed this reduction in noise to a difference in the tail rotor inflow when the main rotor was present, however, they also found no real presence of noise being generated by the main rotor wake/tail rotor interaction. This was not surprising given that the tail rotor under investigation was of the ASD configuration and that special care was taken to minimize the strength of the main rotor tip vortices (so as to minimize main rotor BVI). 
Additional model studies were conducted by Schultz et al ${ }^{16}$ in the DNW wind tunnel that used a heavily instrumented $40 \%$ scale BO-105 model to explore the presence and characteristics of this interaction. The averaged time histories of the isolated model tail rotor and of the combined main/tail rotor was inspected for signs of the main rotor wake/tail rotor interaction. The work reported, "no significant main rotor tip vortex/ tail rotor interference could be identified even by close inspection of both the un-averaged instantaneous blade pressure or sound pressure time histories." Again, this was unsurprising given that the tail rotor of the $\mathrm{BO}-105$ is an ASD rotor.

This experiment was essentially repeated more recently in reference 41 as part of the European HeliNOVI project. The main rotor/tail rotor interaction effects became more discernable with the new data. However, the conclusion was still the same for this model scale BO-105 helicopter rig - the noise due to the main rotor/tail rotor interaction was small at best. Tail rotor noise for the BO-105 helicopter was dominated by loading and thickness noise in addition to BVI on the tail rotor. Unfortunately, the main rotor and tail rotor model was not geared together as it is on the real helicopter - a limitation that forced the researchers to use conditioned sampling to arrive at their results.

The resultant contradiction from the body of work related to tail rotor acoustics has increased the need for a more thorough investigation of the noise mechanisms involved during full-scale helicopter flight. Clearly the use of an ASD rotor plays a large role in the importance of this interaction noise. However, more must be done to study the phenomenon at full-scale under controlled non-dimensional test conditions. The importance of the use of full-scale testing stems from the belief by some that the studies which found little evidence of the main rotor wake/tail rotor interaction were as a direct 
result of scaling issues and that under some conditions an ASD rotor might result in interaction noise. $^{8}$

It is also pertinent at this point to mention that the majority of the tail rotor noise research in forward flight had been performed in acoustically treated wind tunnels on relatively small-scale models. In the wind tunnel environment, time and frequency averaging techniques were often used to help extract the periodic harmonic noise from the other non-periodic sources of noise. A fixed distance between the noise source and measurement microphone enables the use of time averaging methods. It is also of significance to note that while some of the model testing that has been conducted to explore the tail rotor as a noise source has examined time histories of the acoustic pressure, the majority of the work done on full-scale helicopters has been done in the frequency domain.

Time averaging is difficult when using ground based noise measurements (for full-scale helicopter flight testing) due to the Doppler effect - the change in the source-tomicrophone location as a function of time (Figure 1.11 (a)). This change in distance requires a de-Dopplerization procedure in order to perform time averaging, a process which requires extremely precise knowledge of the helicopters location and orientation. This often forces the use of a frequency domain analysis for full-scale flight-testing. By observing data only in the frequency domain a great deal of information can be lost with respect to the physical mechanisms associated with the noise. Time domain averaging provides valuable insight into the directivity of certain noise sources as well as phasing of the sources. 
Another method of measuring helicopter impulsive noise is to fly the measurement microphone at a fixed position with respect to the helicopter. A significant amount of success has been achieved in the past for studying main rotor noise using a "quiet" aircraft (YO-3A) with attached microphones to measure the impulsive noise sources (Figure 1.11 (b)) ${ }^{24,25,26}$ However, because it is impossible to hold the distance between the microphone and the noise source constant, neither the main rotor nor the tail rotor 1/rev signal can be used to time average the acoustic signals. Random errors in the distance between the acoustic source and the microphone translate into errors in the triggering signal, eliminating the possibility of using a one-per-rev trigger pulse that is related to the aerodynamic events on the rotor. Instead time averaging is performed using repeatable acoustic events measured by the microphone.

A good way of minimizing the time errors associated with the changing distances between the measurement microphone and the noise source is to simply fix the microphone to the helicopter (Figure 1.11 (c)). The acoustic signal can now be "phase locked" with respect to the rotor - so that periodic events associated with either rotor system can be extracted from the overall noise radiated by the helicopter. 


\section{a) Ground Based Measurement}

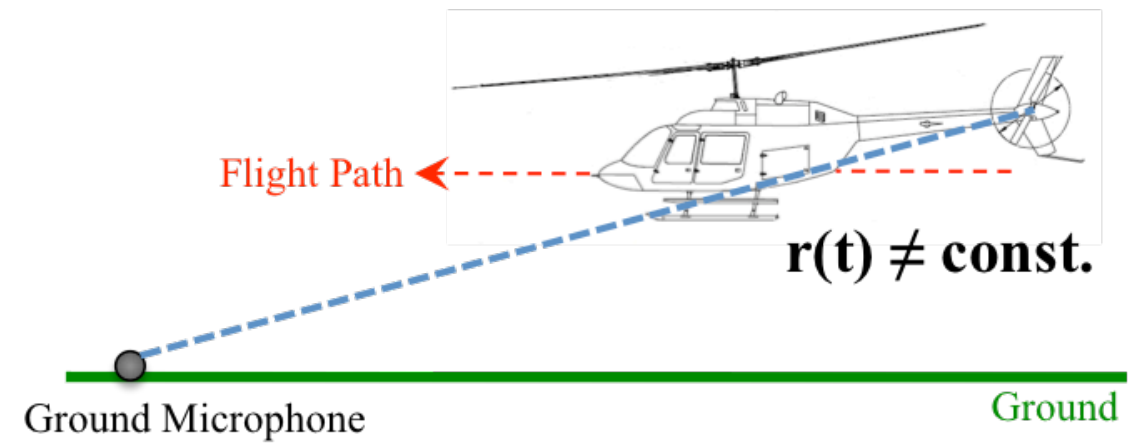

b) Leader Aircraft Measurement

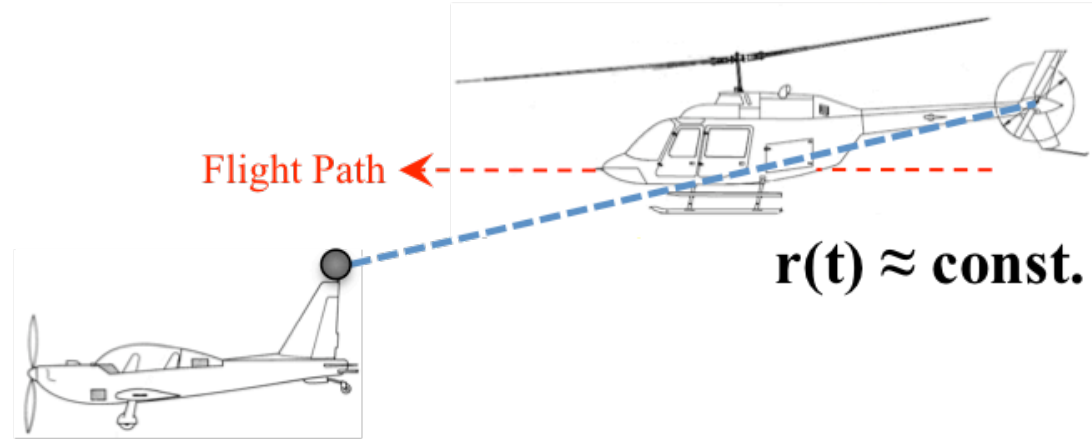

c) Helicopter Mounted Measurement

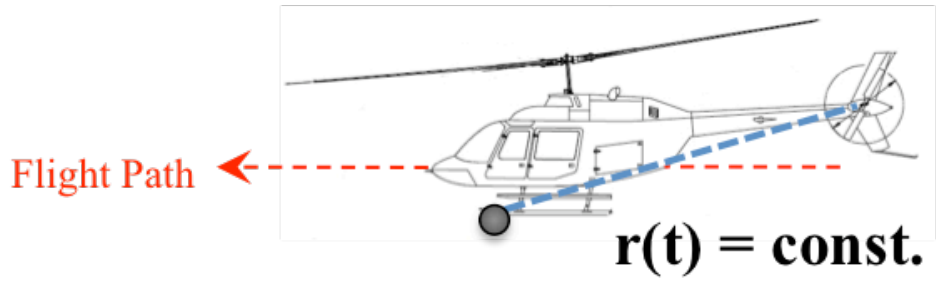

Figure 1.11: Difference in Full-scale Noise measurement techniques

\subsection{Research Objectives}

The ultimate goal of this research is to develop a method with which full-scale tail rotor noise can be examined in the time domain with the ultimate goal of obtaining a better understanding of the tail rotor as a noise source. The specific research objectives are as follows: 
- To develop a new in-flight method that uses a flying array of microphones fixed to the helicopter to investigate the acoustics of a full-scale tail rotor.

- Utilize a major advantage of this method to look at tail rotor noise - using time averaging techniques to extract the tail rotor time histories.

- To investigate the characteristics and dominant sources of tail rotor noise utilizing this new in-flight approach.

These objectives are elaborated upon below.

\section{Development of an In-Flight Measurement Method}

In order to get around the changing position problem associated with most full-scale testing, the microphones must fly with the helicopter at fixed positions with respect to the tail rotor so as to exploit the periodic nature of the tail rotor (and thus allow time averaging). The University of Maryland Boom Microphone array ${ }^{18}$ has been used to obtain acoustic time histories of the noise of a Bell 206B, during both forward and descending flight, in the fixed frame of the helicopter. The boom-mounted microphones are located in the acoustic far-field of the tail rotor on this helicopter and provide the opportunity for a first look at the directivity of the tail rotor noise as a function of flight condition.

\section{Extraction of Tail Rotor Time Histories using Time Averaging Techniques}

Using data taken during a 2006 flight test, a joint program between the University of Maryland, the Army, and NASA, the time averaging method has been developed and explored for the Bell 206B helicopter under level and descending flight conditions. This process involved the use of the physical gear ratio between the main transmission and tail 
rotor to effectively focus in on the periodic noise associated with the tail rotor. Because main rotor and tail rotor noise are aperiodic with respect to each other, the use of an averaging technique allowed for the "extraction" of tail rotor noise time histories.

\section{Attain a Better Understanding of the Characteristics and Dominant Sources of Tail Rotor Noise}

Using the time averaged acoustic histories, this thesis will explore the trends that can be determined for this helicopter under various conditions, as well as compare the results with linear thickness and loading noise theory in the time and frequency domains. The

measured deviation from the averaging will be presented and discussed, showing the possible existence of other apparent aperiodic noise - possibly due to unsteady flight conditions, main rotor wake/tail rotor interaction or atmospheric turbulence.

\subsection{Outline of Thesis}

The significance of the tail rotor as an acoustic source was explored in this first chapter. In addition to an exploration of the fundamental noise mechanisms associated with rotors, a thorough review of the tail rotor acoustic literature revealed that some discrepancy existed in the acoustic community. While some believe that the predominant source of tail rotor noise is simply the result of classical thickness and loading noise, others have concluded that it is actually the interaction between the main rotor wake and tail rotor that is responsible for most of the noise emanated by tail rotors. This rift in conventional thinking highlights the need for a better understanding of tail rotor noise, specifically through the use of full-scale acoustic analysis in the time and frequency domain. This 
first chapter has aimed to emphasize the importance of this research and provide the necessary background for a proper understanding of this new approach.

The following chapter explains the experimental method that was used, specifically discussing the various techniques and instruments that were used during the 2006 CRI and 2008 Flight Tests conducted at Moffett Field, CA. Additionally, a description of the tail rotor of the Bell 206B helicopter is provided. Chapter 3 focuses on what was done with the acoustic data after it had been gathered. After commenting on how useful a time domain analysis can be, the specific details of the time averaging method used in this work are given. Chapter 4 presents the time-averaged results obtained for the Bell 206B during both steady level and descending flight. The trends in directivity for both flight conditions are given.

Beyond providing the time averaged acoustic histories for the tail rotor a full comparison with linear thickness and loading noise theory was made so as to better understand what is seen in the time averages. Chapter 5 discusses the theoretical tail rotor model used for this purpose, including both the thickness and loading implementation of the Ffowcs Williams and Hawkings equations. Chapter 6 then shows the comparison of this theoretical model with the time average results explored in Chapter 4.

Finally, Chapter 7 and 8 explore any additional considerations that might be made regarding the existence of additional acoustic energy not captured by the averaging process and discuss the conclusions that can be drawn from the research. Future research ideas are also presented. 


\section{Chapter 2 Flight Testing}

\subsection{Summary}

This chapter describes the experimental flight testing approach used to gather the tail rotor noise data used in this thesis. It was gathered in a CRI/NASA/Army/University of Maryland flight test that was conducted in the summer of 2006. The details of an additional test, conducted in the spring of 2008, are also discussed. Data taken during these flight tests accounts for the entirety of the flight data used in this research.

In addition to the details of the flight tests, this chapter will also discuss the physical and major mechanical attributes of the Bell 206B helicopter that was used in both tests. The Bell 206B has a single main rotor and a conventional, pusher type, ASD tail rotor.

Figure 2.1 shows major dimensions of the Bell 206B helicopter.

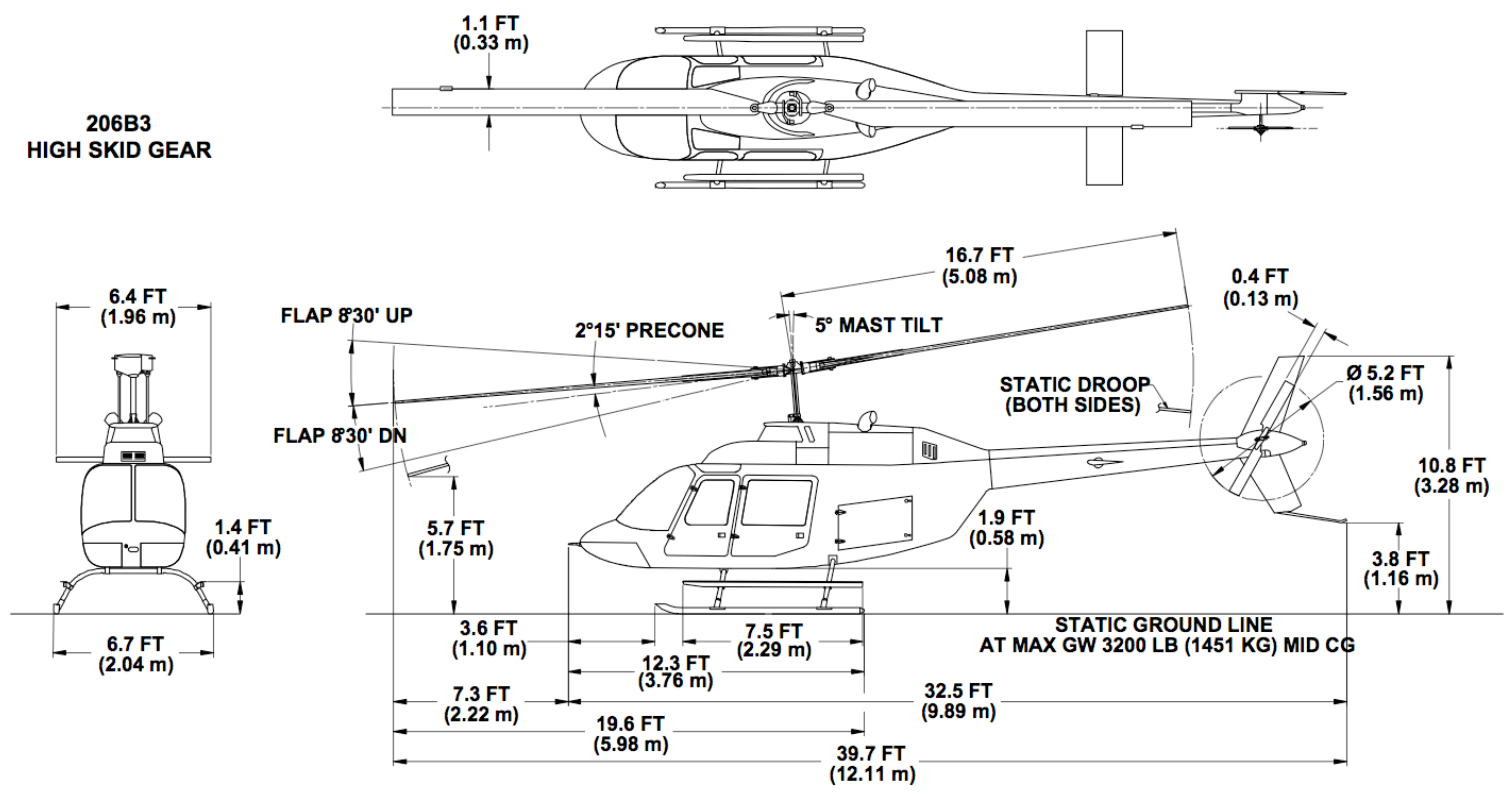

Figure 2.1: Exterior dimensions of the Bell $206 \mathrm{B3}$ helicopter $^{20}$ 


\subsection{Methodology}

In order to obtain full-scale time averaged acoustic histories of the tail rotor of the Bell 206B during a variety of flight maneuvers an in-flight measurement scheme was used with the microphones physically attached to the reference frame of the helicopter.

\subsection{June 2006 Flight Test}

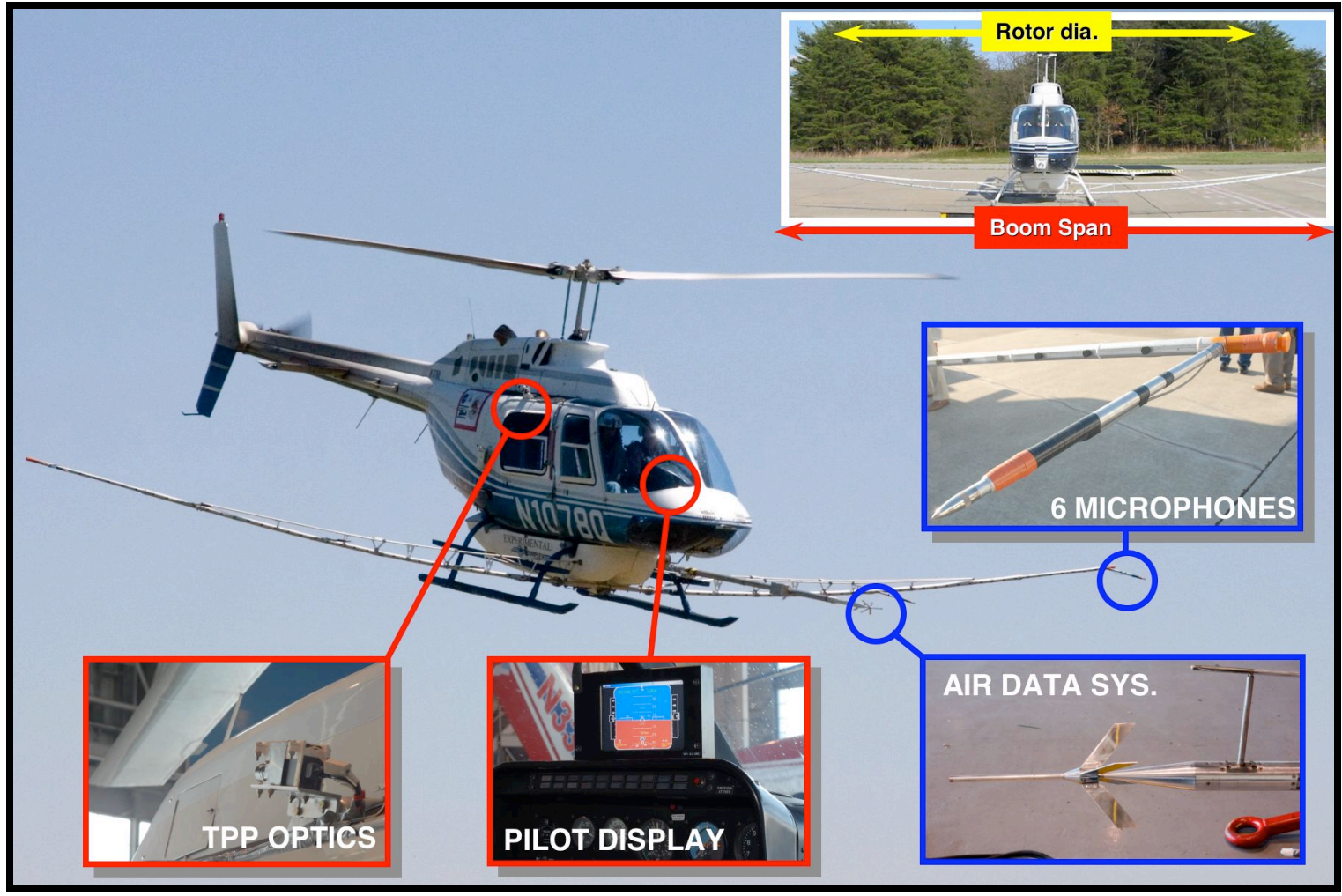

Figure 2.2: 2006 flight test instrumentation

In June of 2006 a joint program between the Center for Rotorcraft Innovation (CRI), NASA, the Army and the University of Maryland was conducted at Moffett field, CA. The primary goal of this program was to measure the radiated noise of the main rotor of a Bell 206B helicopter during a variety of flight conditions. Special attention was given to Blade-Vortex Interaction (BVI) of the main rotor. The current research focuses 
exclusively on the in-flight data obtained for both level and descending flight. Additional work published using this data set can be found in reference 19 .

During the test, a Bell 206B helicopter was outfitted with a series of instruments designed for full-scale acoustics testing: an air data system, a main rotor 1/rev sensor, a pilot display system, an experimental tip-path plane (TPP) optical system, and a modified commercial spray rig used for crop dusting, outfitted with six microphones.

\subsubsection{Air Data System}

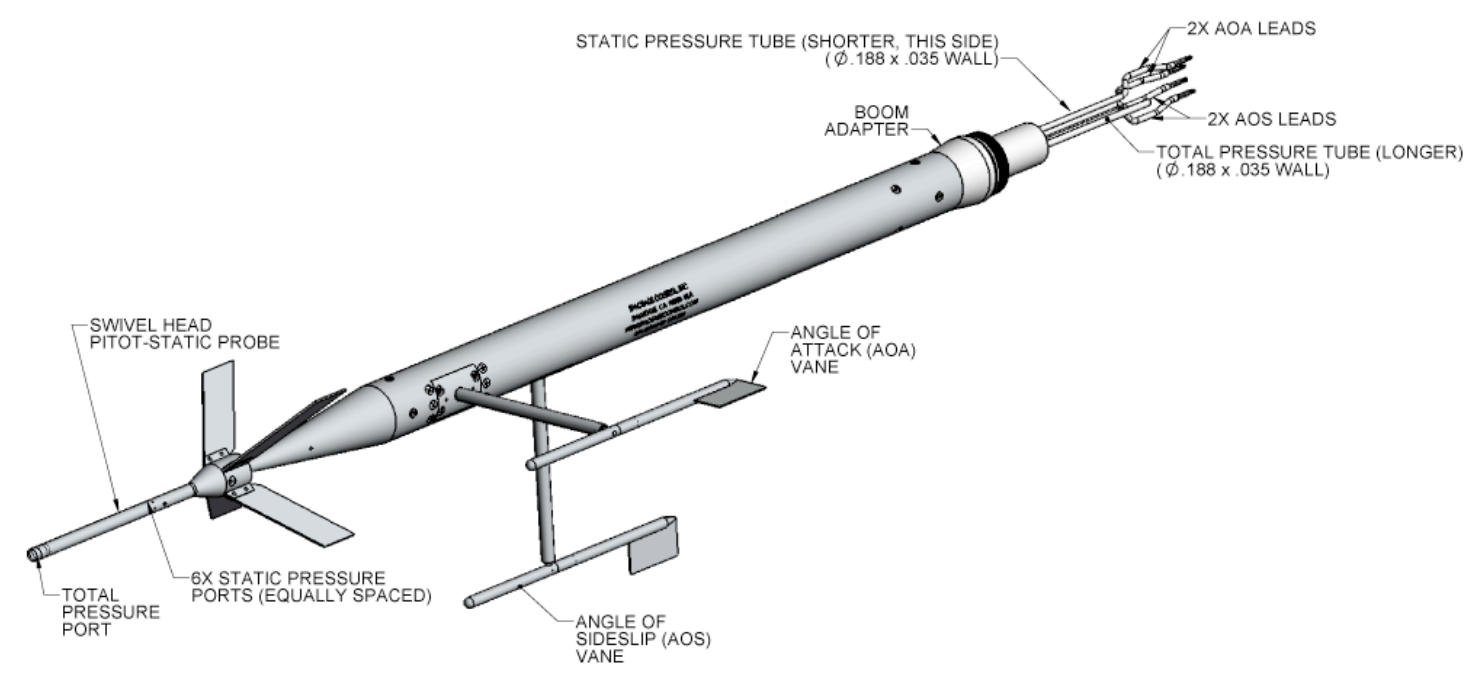

Figure 2.3: Space Age Control 100510 Swivel-Head air data system

The air data system used for this flight test consisted of three major components: the boom, the instrument pod, and the swivel-head air data system. The boom was constructed from aluminum and designed to extend beyond the wake of the main rotor. This allowed for precise measurement of the relative velocity of the aircraft and the angle of attack and sideslip of the helicopter fuselage. Special care was taken in the design of 
this boom so as to minimize the transmission of any rotor born vibrations. The boom was attached to the skids of the helicopter, as seen in Figure 2.2.

The instrument pod, located near the front end of the boom, contained the transducers required for proper operation of the air data system. An additional thermocouple was used so as to provide a measurement of the air temperature during testing.

The third and final component of the air data boom was the Space Age Control 100510 swivel-head air data system, shown in Figure 2.3. This system measured the airspeed, fuselage angle of attack and sideslip of the helicopter with respect to a reference line on the helicopter. The data obtained with this boom was transmitted to the Vision Data Recording system held and operated within the helicopter cabin.

\subsubsection{Main Rotor One-Per-Rev Sensor}

The availability of a main rotor one-per-rev sensor for this flight test facilitated the use of time averaging to extract the time domain characteristics of the main rotor of the Bell 206B. Though a tail rotor one-per-rev sensor would have made time averaging for extraction of the tail rotor characteristics easier, knowledge of the main rotor azimuth angle and the gear ratio between the main and tail rotor allowed for the generation of a theoretical tail rotor one-per-rev. The specifics of this gear ratio are discussed further in Section 2.4

A Shimpo 3030AN Magnetic Proximity Sensor was used for detection of the main rotor revolution rate, which was sampled at $20 \mathrm{kHz}$ by the onboard Vision Data Measurement system. Because the average rotational frequency of the main rotor of the Bell 206B is $6.5 \mathrm{~Hz}$, the $20 \mathrm{kHz}$ sampling rate is equivalent to an angular measurement resolution of 
roughly $0.117^{\circ}$ or an error of $\pm 0.0021 \mathrm{~Hz}$ in the rotational frequency measurement of the main rotor.

\subsubsection{Pilot Display System}

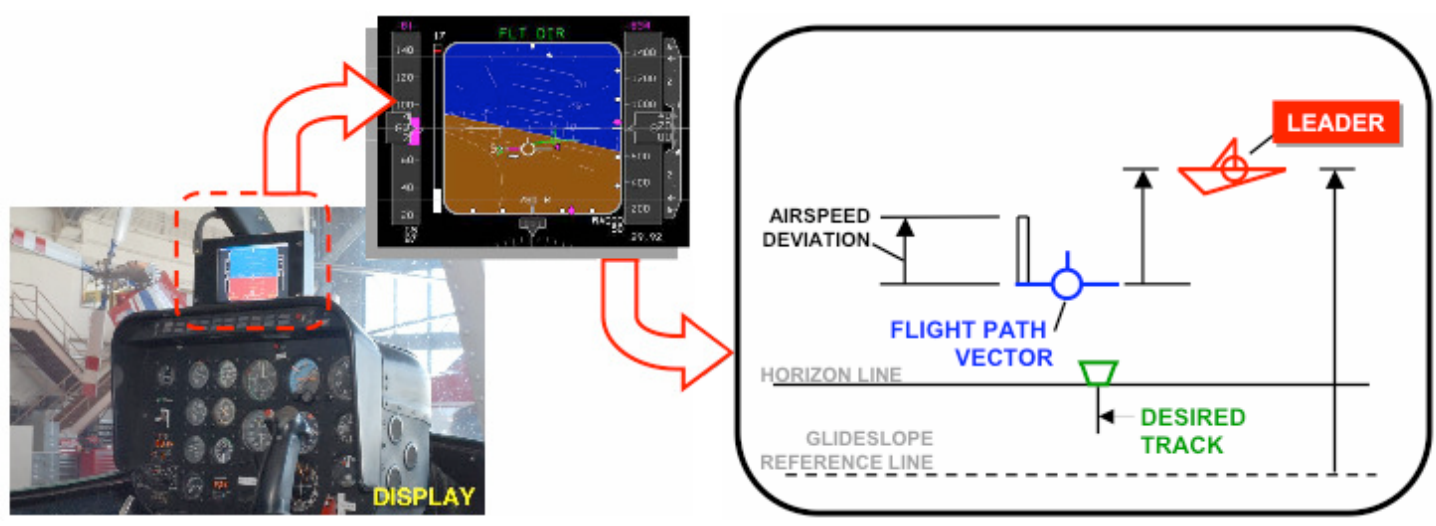

Figure 2.4: The PPDG display mounted on the Bell 206B ${ }^{19}$

In order to generate a useful data set, it was imperative that a full understanding of the helicopter flight condition be obtained and that the pilot be able to maintain a given steady flight condition (in this case, steady level flight and descending flight). This was achieved through the use of a NASA developed "Portable Programmable Guidance Display (PPGD)" system for precision pursuit guidance. This system provides the pilot with visual pursuit guidance information for a given flight condition. Figure 2.4 shows this system as it was mounted in the Bell 206B, as well as a simplified schematic of the pursuit display.

Pursuit systems of this nature have been shown to improve the pilot's tracking ability while smoothing out the pilot's inputs. This results in a reduced workload and stick agitation. ${ }^{19,21}$ The pilot tracks the "leader aircraft" (shown in red) by flying his aircraft (shown in blue) to the same position, velocity, and acceleration of the leader. By using 
this system, the quality of the acoustic measurements was greatly improved. Tail rotor noise, as any noise, can be extremely sensitive to small but abrupt adjustments in pedal condition or fuselage orientation. Minimization of the perturbations in these variables was thought to improve the quality of the data.

The PPGD was coupled with airspeed information obtained from the air data system, as well as an inertial navigation unit (INU) and differential global positioning system (DGPS).

\subsubsection{University of Maryland Boom Microphone Array}

As discussed previously, the primary goal of this flight test was to study and measure main rotor Blade-Vortex Interaction (BVI). In order to measure this noise in-flight, a commercial crop dusting spray rig, originally developed for use in crop dusting, was attached to the underside of the helicopter and outfitted with six Bruel \& Kjaer microphones (two $1 / 4$ in, four $1 / 2$ in) fitted with wind shielding nose cones (see Appendix A for more details). The placement of these microphones was such that it helped assess the radiation patterns of BVI noise, with four microphones on the helicopter main rotor's advancing side (starboard) and two on the retreating side (port), as shown in Figure 2.5. This system has been used successfully in several flight tests ${ }^{18,19}$ to obtain important information concerning the directivity and amplitude of main rotor noise during various flight maneuvers.

As a means of referring to the microphone data throughout this report, a simple naming scheme was derived. The four microphones on the advancing side of the helicopter were designated A1, A2, A3 and A4, while the two retreating side microphones were 
designated R1 and R2. The lower number microphones correspond to the microphones closest to the tip-path plane of the tail rotor (which is located on the port side of the helicopter tail boom), while the larger number microphones are located further out of plane. This placement provides a wide sweep of microphone locations, ideal for an investigation of tail rotor noise. The positions of these microphones with respect to the tail rotor hub are summarized in Table 2.1 and the coordinate systems with which these values correspond are shown in Figure 2.6.

Table 2.1: Microphone positions

\begin{tabular}{ccccccc}
\hline \hline Microphone & $\boldsymbol{X}(\boldsymbol{T R}) \boldsymbol{f t}$ & $\boldsymbol{Y}(\boldsymbol{T R}) \boldsymbol{f t}$ & $\boldsymbol{Z}(\boldsymbol{T R}) \boldsymbol{f t}$ & $\boldsymbol{\Psi}$ & $\boldsymbol{\Phi}$ & $\boldsymbol{T R}$ Radii \\
\hline A4 (Apple) & 23.74 & 22.29 & 4.663 & $169.9^{\circ}$ & $43.20^{\circ}$ & 12.65 \\
A3 (Orange) & 23.74 & 17.25 & 5.393 & $167.2^{\circ}$ & $36.01^{\circ}$ & 11.48 \\
A2 (Peach) & 23.74 & 12.21 & 6.123 & $165.5^{\circ}$ & $27.22^{\circ}$ & 10.53 \\
A1 (Kiwi) & 23.74 & 7.172 & 6.693 & $164.3^{\circ}$ & $16.81^{\circ}$ & 9.88 \\
R1 (Grape) & 23.74 & -10.51 & 6.143 & $165.5^{\circ}$ & $-23.88^{\circ}$ & 10.26 \\
R2 (Lime) & 23.74 & -20.57 & 4.753 & $168.7^{\circ}$ & $-40.91^{\circ}$ & 12.22 \\
\hline \hline
\end{tabular}

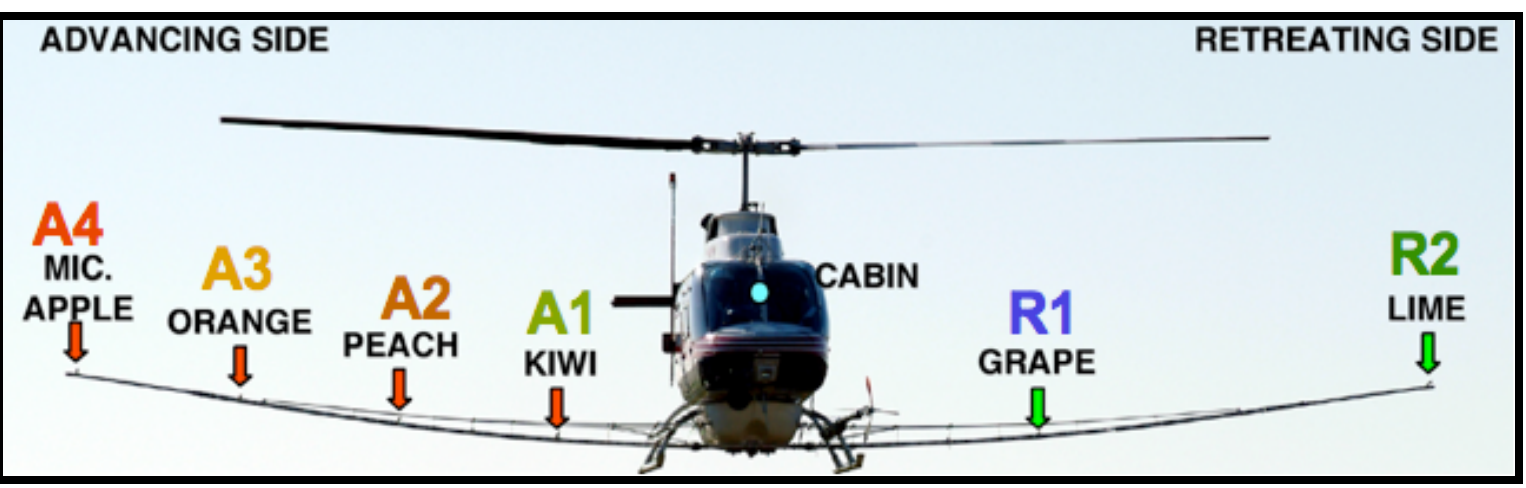

Figure 2.5: Mic. locations on UMD Boom Microphone Array 


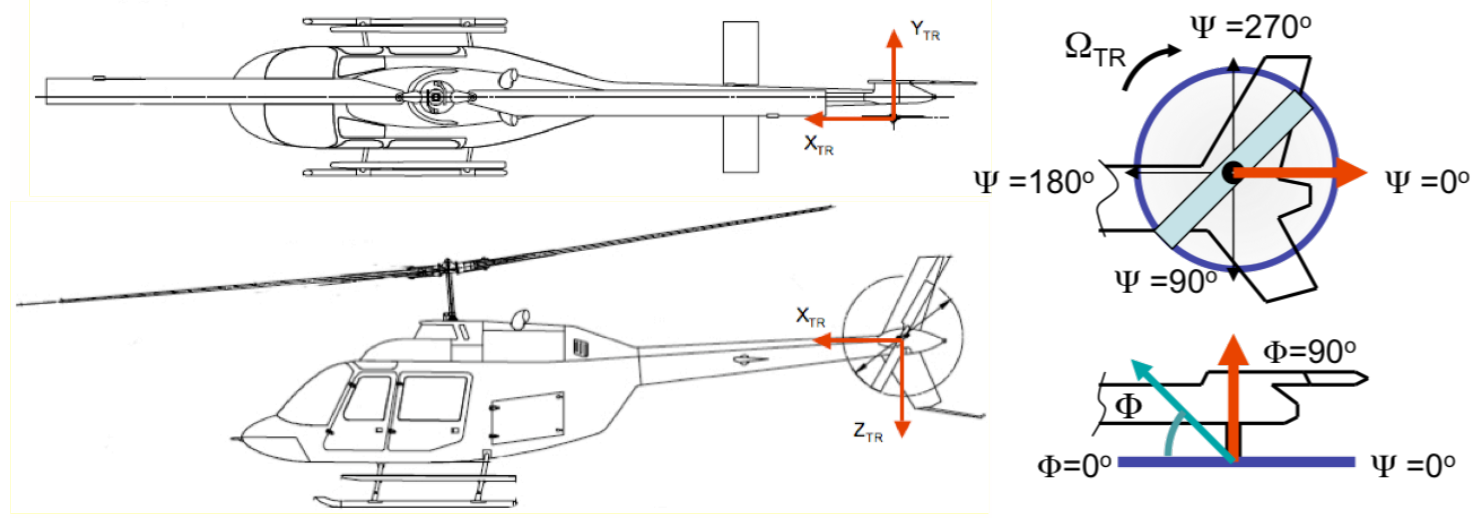

Figure 2.6: Coordinate system definition for Bell 206B tail rotor

The microphones are located at angles ranging from approximately $43.20^{\circ}$ above the rotor (in the direction that thrust is being generated) to $40.91^{\circ}$ below the rotor, all at tail rotor azimuth angles of between $164.3^{\circ}$ and $169.9^{\circ}$. Additionally, all microphones are located a distance of 9.88 tail rotor radii $(2.6 \mathrm{ft})$ or further away. This fact allows for a comparison of the acoustic pressures recorded by each microphone that have been scaled to a distance of 10 tail rotor radii from the tail rotor hub (26 ft). This is a technique that is used frequently in acoustics research ${ }^{22}$ and allows for a good understanding of the directivity of the noise being generated. An illustration of the sphere onto which the microphone acoustic data is scaled is shown in Figure 2.7.

The benefits of using a microphone array that is moving with the helicopter, as opposed to just ground microphones, are many and are discussed in Chapter 3. Other benefits include the ability to observe the noise produced by a tail rotor of any configuration, including shrouded, pusher or puller tail rotors. The only real negative consequence of a system of this nature is that the flight capabilities of the helicopter are compromised by the additional drag created by the boom, limiting the maximum speed or climb rates of the aircraft. 


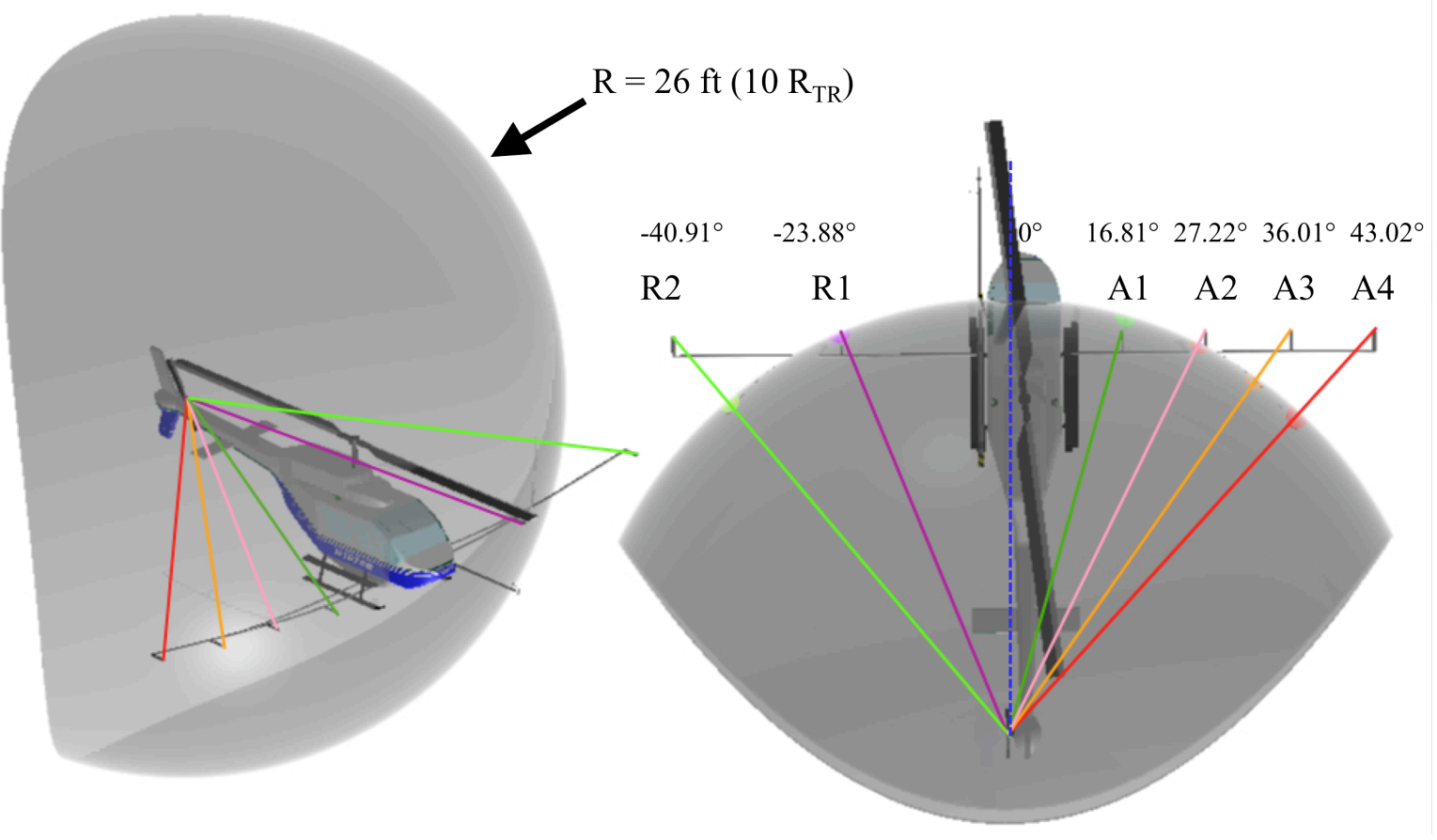

Figure 2.7: Sphere representing scaling used for acoustic measurements

\subsubsection{Meteorological Conditions}

The majority of the flights for this flight test were conducted early in the morning when the wind conditions were low (less than 5 knots). This was done because horizontal wind and wind gradients have been shown to reduce the quality of noise measurements. ${ }^{19}$ Though this is less of a concern with microphones moving with the helicopter, it is still true that a steady flight condition is more easily obtained in a low wind environment.

During the test, a NASA tethered weather balloon system measured the ambient wind speed and direction, temperature, and humidity profiles every half hour. An example of this balloon data for a standard day is shown in Figure 2.8. 

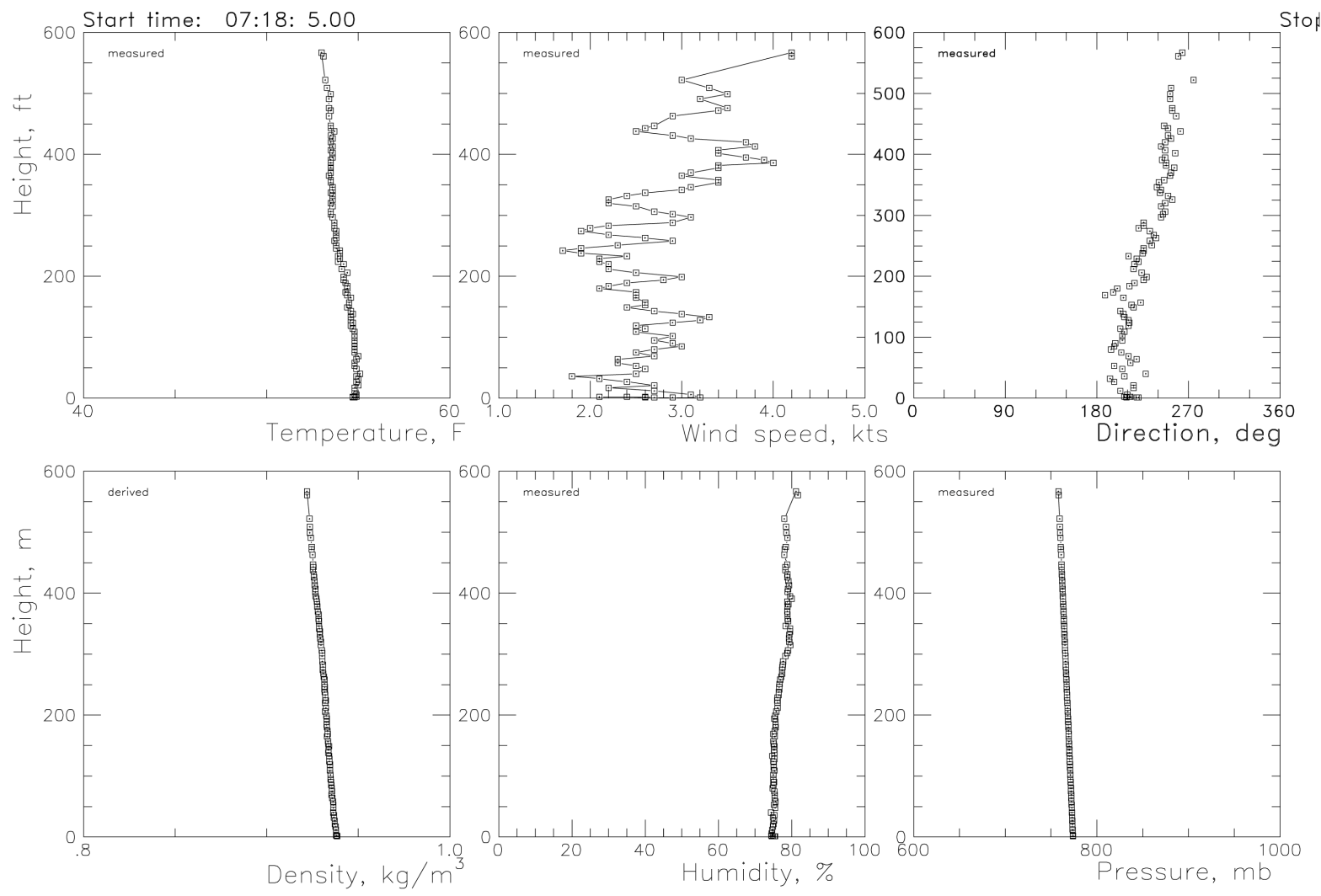

Figure 2.8: Typical weather balloon data (June 14, 2006)

\subsubsection{Test Conditions}

Though a considerable number of runs were made during this flight test, including acceleration and decelerations, steady turns, transient maneuvers, level flight, and steady descents, only ten cases were focused upon for this research. These included four steady level flights, and six steady descents. Though a large number of runs were made, the best were chosen based upon the flight steadiness, velocity, and wind conditions. The resultant data set used is summarized in Table 2.2. 
Table 2.2: Summary of Flight Conditions

\begin{tabular}{ccc}
\hline \hline Case (Data Set) & Velocity [knots] & Descent Angle [degrees] \\
\hline Case 1 (142) & 38 & 0 \\
Case 2 (128) & 53 & 0 \\
Case 3 (136) & 62 & 0 \\
Case 4 (144) & 70 & 0 \\
Case 5 (211) & 63 & 0 \\
Case 6 (212) & 63 & -3 \\
Case 7 (214) & 63 & -6 \\
Case 8 (215) & 63 & -7.5 \\
Case 9 (216) & 63 & -9 \\
Case 10 (219) & 63 & -12 \\
\hline \hline
\end{tabular}

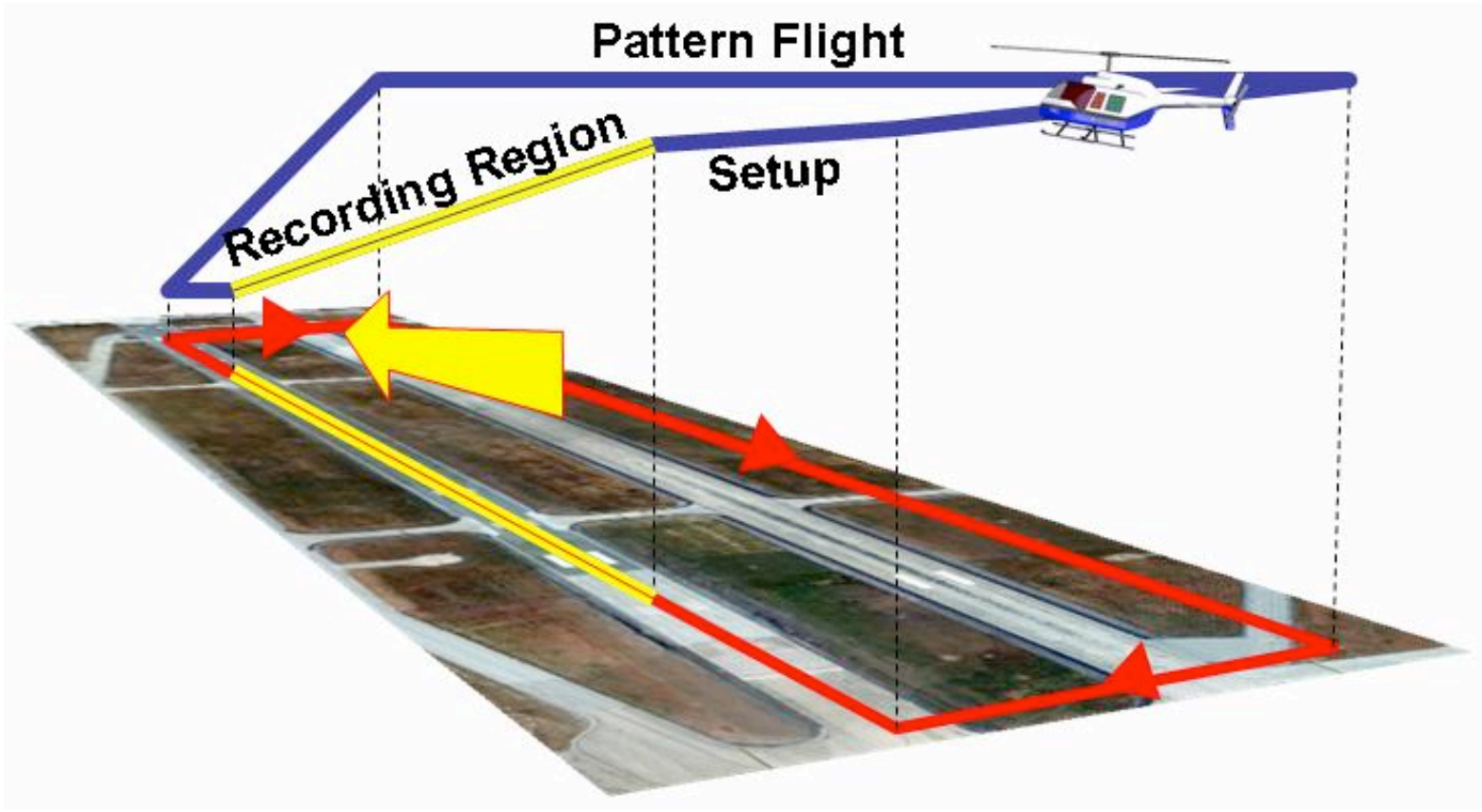

Figure 2.9: Flight testing procedure at Moffett Field, CA

The general flight procedure for this test included three major steps. Because the flight test was conducted at Moffett Field, an operating airfield, special care was given to obeying the regulations set fourth by NASA and the Moffett Field Air Control Tower. 
Each flight run began by flying in pattern to the designated setup point, at which time the pilot maneuvered the helicopter into the pre-designated starting point and flight condition. This point was based on the location of the ground microphones and the setup condition varied in altitude and/or velocity. Data was turned on and collected through the entire maneuver, then turned off at a distance downfield of the microphones. This pattern is illustrated in Figure 2.9.

\subsection{March 2008 Flight Test}

In March of 2008, an additional flight test was conducted so as to validate the rotation ratio between the main rotor and tail rotor of the helicopter. Because a tail rotor one-perrev was not used in the initial 2007 test, the revolutions of the tail rotor were simulated based on the main rotor rpm. In theory, knowledge of the physical gear ratio between the main transmission and the tail rotor should provide adequate information so as to generate this theoretical signal. However, it was unclear as to whether there might be significant "wiggle" or "slippage" within the transmission system that would result in an erroneous prediction of the tail rotor rpm. This would then result in poor tail rotor time averages.

The gear ratio of 6.472, provided by Bell, was validated during this flight test. In order to validate the ratio, a series of flights were made. These included level, descending, ascending, turning and auto-rotational flight.

In addition to the Shimpo 3030AN Magnetic Proximity Sensor that measured the main rotor rpm, a commercially available infrared sensor, with retro-reflective tape on the blade, was used to measure the tail rotor rpm. Both the main rotor and tail rotor signals 
were then recorded at $20 \mathrm{kHz}$ using the Vision Data System (equivalent to $0.12^{\circ}$ angular resolution or $\pm 0.0021 \mathrm{~Hz}$ for the main rotor and $0.75^{\circ}$ angular resolution or $\pm 0.088 \mathrm{~Hz}$ for the tail rotor).

In order to extract the gear ratio, a program was written in MATLAB that conditioned the raw one-per-rev data, calculated the rotor rotation period for both the main and tail rotor, and then divided the two to obtain the transmission gear ratio. Because the sensor for both rotors was a one-per-rev, and not a digital encoder that would provide information concerning slight changes in rpm within a single revolution, it was assumed that the rpm stayed constant over a given revolution. The validity of this assumption is explored further in Chapter 3. This process is depicted in Figure 2.10 (a) to (e) where Figure 2.10 (a) and (b) show the conditioned $1 /$ rev data for the main and tail rotors respectively, Figure 2.10 (c) and (d) show the calculated periods, and Figure 2.10 (e) shows the resultant gear ratio.

In order to ascertain if this gear ratio was constant during the flight maneuvers under examination, so that a gear ratio of 6.472 could be used regardless of the flight condition, the entire dataset was combined so as to obtain over six million samples. Figure 2.11 shows that the resultant data can be represented by a normal Gaussian distribution centered on 6.472. Furthermore, the standard deviation of this data is 0.008 , which has an equivalent $2 \sigma$ error of 0.16 . This deviation is very close to the error that is expected when considering the accuracy of both the main rotor and tail rotor one-per-rev measurements. If for example there is a main rotor rotational frequency of $6.5 \pm 0.0021$ $\mathrm{Hz}$ and a tail rotor rotational frequency of $42.068 \pm 0.0881 \mathrm{~Hz}$ (where $42.068=\Omega_{\mathrm{MR}} \times$ Gear Ratio), then the resultant gear ratio that can be calculated based on these 
measurements is $6.472 \pm 0.016$. Because the standard deviation is of the same order as the error associated with the measurement techniques, it was assumed that a gear ratio of 6.472 was maintained throughout the flight envelope

a) Main Rotor 1/REV

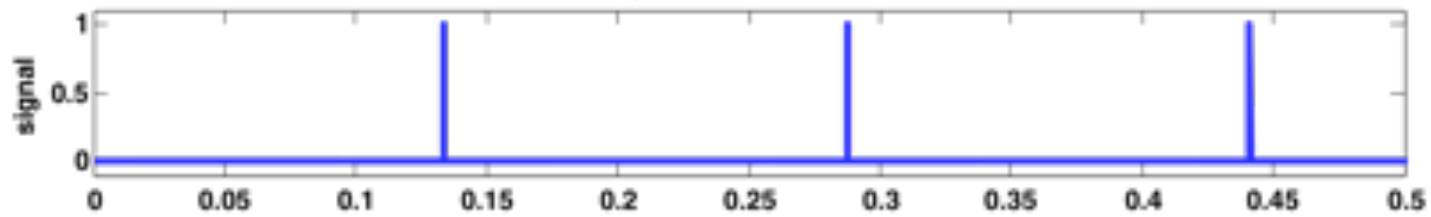

b) Tail Rotor 1/REV

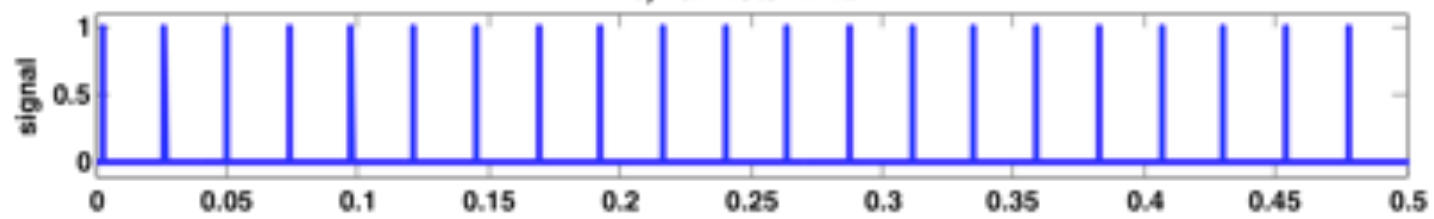

c) Main Rotor Period
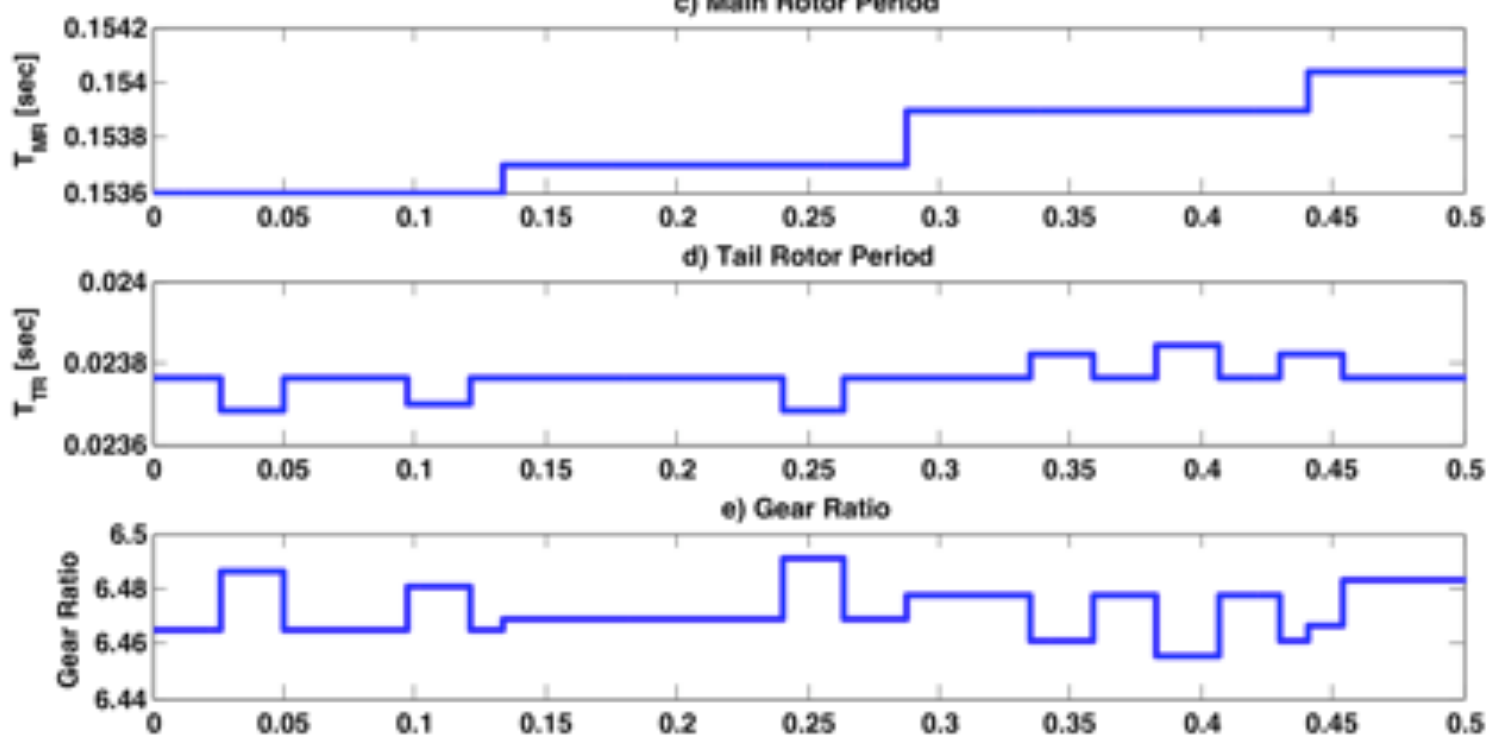

Figure 2.10: Method for the determination of main rotor/tail rotor gear ratio 


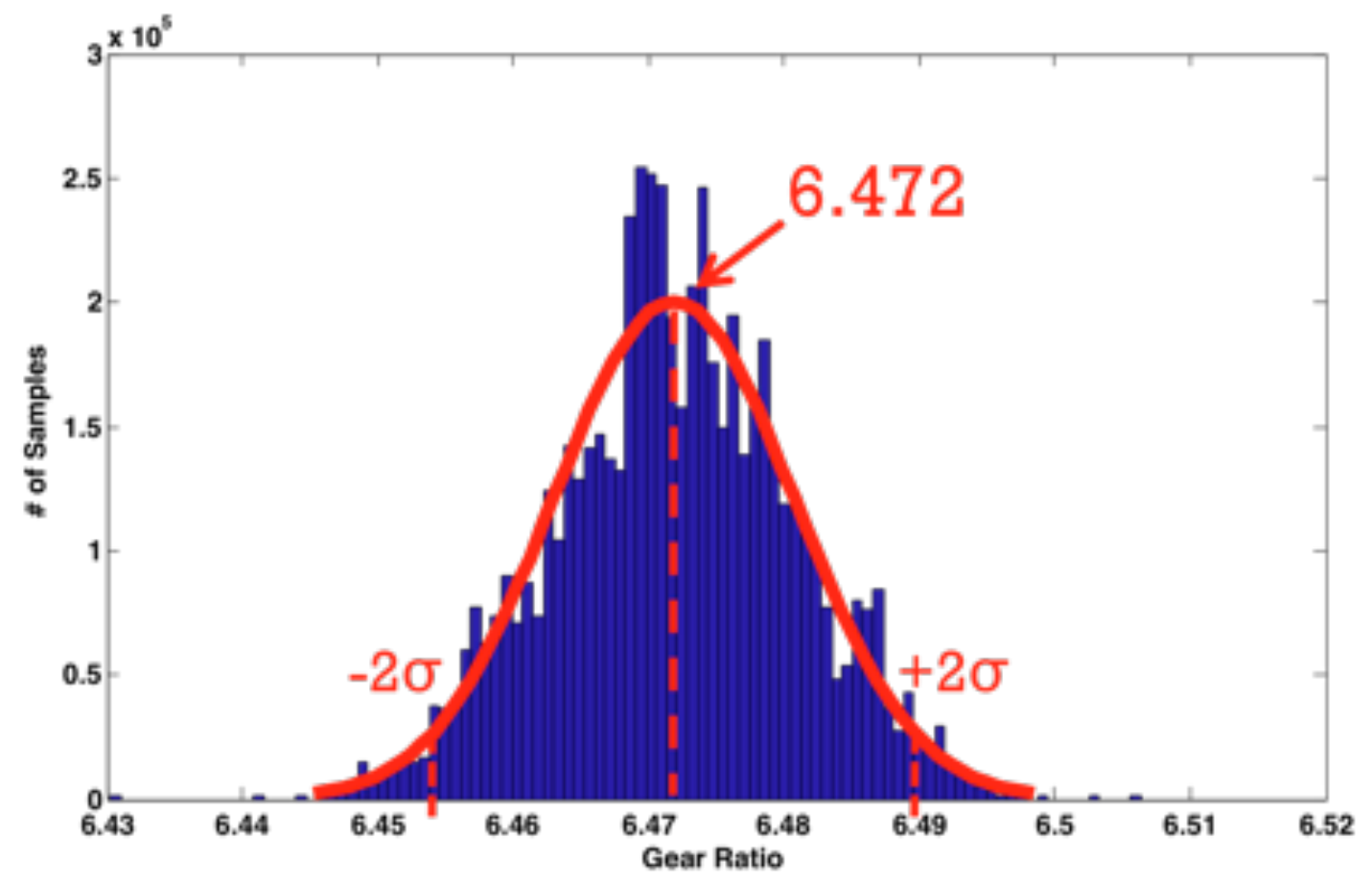

Figure 2.11: Main rotor to tail rotor gear ratio statistical data

\subsection{The Tail Rotor of the Bell 206B}

In addition to the methods and results of the flight tests, a full understanding of the configuration and geometry of the tail rotor in question is crucial to a full understanding of the harmonic noise it produces. As discussed in Chapter 1, previous work in the field of tail rotor acoustics has concluded that factors as simple as the direction of rotation can drastically alter the noise generated by a tail rotor. ${ }^{8}$ Therefore it is imperative that the reader understand that the conclusions drawn in this research are applicable only to the tail rotor of the Bell 206B, or similarly configured and sized tail rotors. More general conclusions concerning tail rotors' as a whole would require an examination of other tail rotor configurations and their respective acoustic measurements. 


\subsubsection{Tail Rotor Configuration}

The primary purpose of a tail rotor is to provide a force on the fuselage that is large enough so as to counter the torque generated by the helicopters main rotor. Two major types of conventional tail rotors exist, pushers and tractors.

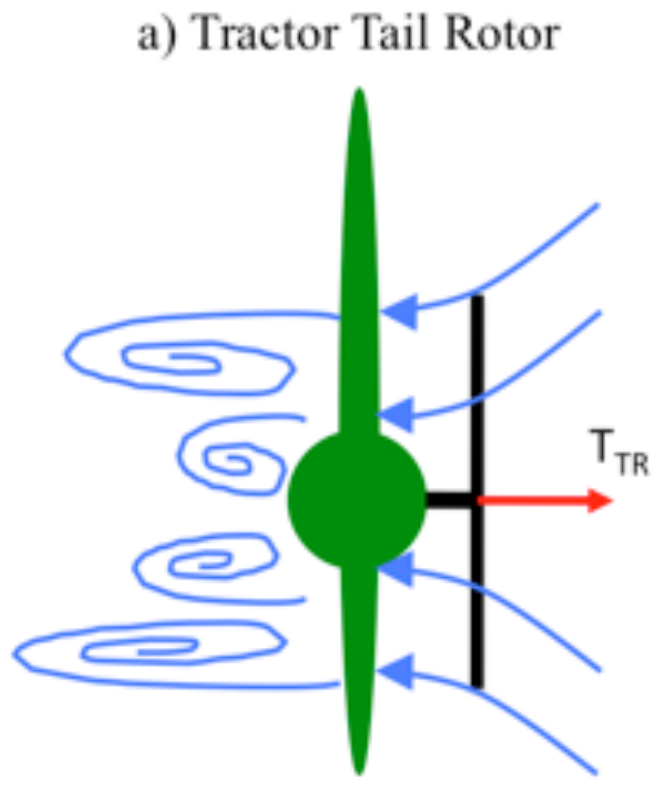

b) Pusher Tail Rotor

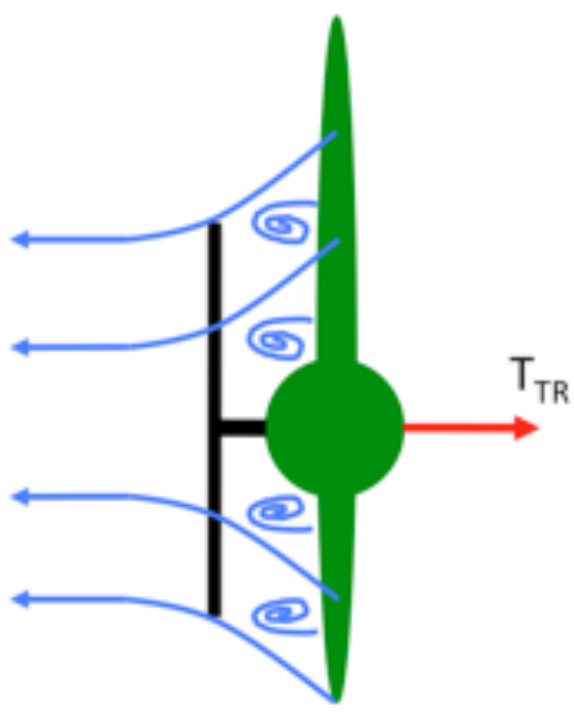

Figure 2.12: Tail rotor configurations a) tractor b) pusher

A tractor tail rotor (Figure 2.12 (a)) locates the vertical fin downstream of the rotor, in its wake. Doing this increases the tail rotor thrust slightly, however a substantial force is applied to the vertical fin in the opposite direction of that required for anti-torque. Generally speaking, a rotor of this configuration leads to a decrease in net thrust when compared to a rotor operating in isolation, or for that matter a pusher tail rotor.

The Bell 206B, as well as the majority of modern helicopters, uses a pusher tail rotor design (Figure 2.12 (b)). In this design, the wake of the tail rotor is convected away from the vertical fin of the helicopter. However, the volume of air that is ingested into the rotor 
experiences a significant amount of turbulence associated with the vertical fin. This results in a non-uniform inflow into the tail rotor as well as a negative pressure generated on the vertical fin. Although the losses associated with this are less than those associated with the tractor design, they do reduce the net thrust when compared to a tail rotor operating in isolation. These effects tend to diminish in forward flight when the tail rotor is required to provide less thrust.

\subsubsection{Direction of Rotation}

As discussed in Chapter 1, the direction of rotation of the tail rotor has been shown to significantly affect its noise, particularly in forward flight. ${ }^{8,23}$ An Advancing Side Up (ASU) rotor, was seen by Leverton et al. to produce a distinctive burble noise on the Westland Lynx helicopter. However, by changing the direction of rotation to an Advancing Side Down (ASD) configuration a significant reduction in this burble noise was found. The Bell 206B has an Advancing Side Down (ASD) tail rotor.

\subsubsection{Tail Rotor Design}

The tail rotor of the Bell 206B is relatively simple in design. It is a two bladed rotor with a teetering hub for flapping flexibility. As with most conventional tail rotors, there is no use of lead-lag hinges. Instead, a considerable amount of pitch-flap $\left(\delta_{3}\right)$ coupling is incorporated into the tail rotor design. This allows the blades to pitch cyclically so as to minimize the amount of flapping that the rotor undergoes. The tail rotor of the 206B has a $\delta_{3}$ angle of $45^{\circ}$. The details of using an analytical model to predict the loading behavior associated with this pitch-flap coupling are discussed in Chapter 5. Because pitch-flap coupling is used, the only input to the tail rotor is the collective, which is transmitted to 
the rotor via a push-pull rod through the center of the tail rotor shaft. The 206B tail rotor is depicted in Figure 2.13.

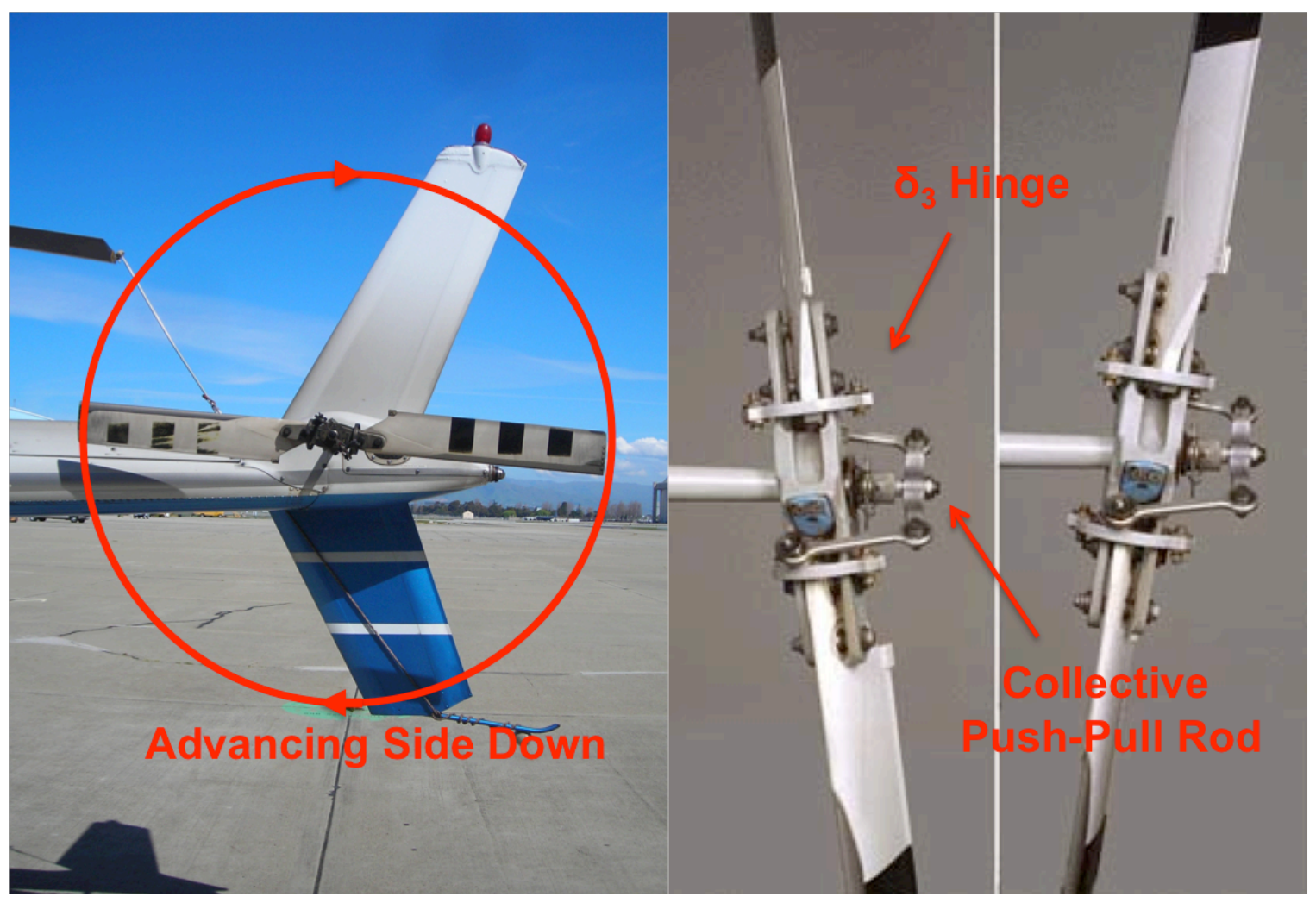

Figure 2.13: The Bell 206B Tail Rotor

\subsubsection{Geometry and Rotation Speed}

The tail rotor of the 206B is very simple in construction. The blades have a symmetrical NACA 0012 airfoil with no twist and a chord of $0.44 \mathrm{ft}$. The rotor diameter is $2.6 \mathrm{ft}$. As discussed in Section 2.4, the tail rotor rotates at 6.472 times the main rotor revolution rate. Given that the average rotation speed of the main rotor is $6.5 \mathrm{~Hz}$, the tail rotor rotates at an average frequency of approximately $41.5 \mathrm{~Hz}$, with a corresponding tip speed of $685 \mathrm{ft} / \mathrm{s}$ which translates to a hovering tip Mach number of the tail rotor of 0.61 . This 
is quite high and results in the high levels of harmonic noise shown in the coming chapters of this thesis.

Table 2.3: Major parameters of the Bell 206B tail rotor

\begin{tabular}{cc}
\hline \hline Radius & $2.6 \mathrm{ft}$ \\
\hline Chord & $0.44 \mathrm{ft}$ \\
\hline Twist & $0 \mathrm{deg}$ \\
\hline $\boldsymbol{\Omega}$ (average) & $41.5 \mathrm{~Hz}(\mathrm{ASD})$ \\
\hline $\mathbf{M}_{\text {HOVER,TIP }}$ & 0.61 \\
\hline Airfoil & NACA 0012 \\
\hline delta-3 & $45 \mathrm{deg}$. \\
\hline
\end{tabular}




\section{Chapter 3 Time Averaging}

\subsection{Summary}

Averaging acoustic data in the frequency domain is a method that has been used for years to study main rotor harmonic noise for both model and full-scale testing. ${ }^{24}$ The process works because harmonic noise occurs at frequencies that are associated with the rotational frequency of the rotor itself and is reinforced in the averaging process. Noise that is not harmonic with respect to the rotation rate of a particular rotor is not reinforced during the averaging process, resulting in a reduction in amplitude relative to the harmonic source under investigation. This can be shown mathematically by examining the average of a pressure signal $\mathrm{P}(\mathrm{t})$ composed of a periodic pressure component $\mathrm{P}_{\mathrm{PER}}(\mathrm{t})$ and an aperiodic noise component, $\mathrm{P}_{\mathrm{APER}, \mathrm{i}}(\mathrm{t})$. Then the average pressure over $\mathrm{R}$ periods is $P_{A V G}(t)=\frac{1}{R} \sum_{i=1}^{R}\left(P_{P E R}+P_{A P E R, i}\right)=\frac{1}{R} \sum_{i=1}^{R} P_{P E R}+\frac{1}{R} \sum_{i=1}^{R} P_{A P E R, i}=P_{P E R}+\frac{1}{R} \sum_{i=1}^{R} P_{A P E R, i}$

where the average of the periodic noise pressure reduces to the total periodic noise, and the average of the aperiodic pressure, which is different for each sample $i$, is reduced by the number of samples used, R. This shows that time-averaging will increase the signal to noise ratio of a given measurement.

Time averaging of acoustic data based upon a periodic marker in the time domain can yield even more information about the periodic stationary noise of a rotor. By maintaining a constant phase throughout the averaging process, harmonic noise that is not an exact multiple of the period of the event being averaged is also removed from the averaged signal. This chapter will discuss in brief the use of this method for the 
examination of simple rotor harmonic noise as well as the additional complications that arise when time averaging for an examination of tail rotor harmonic noise.

\subsection{Main Rotor Time Averaging}

Because the tail rotor of a helicopter generally operates at a non-integer multiple of the main rotor rotational speed, averaging acoustic data in the time domain on the main rotor revolution period, using a $1 /$ rev signal, has proven to be an extremely effective way of isolating the main rotor harmonic noise pulse shape from all other noise sources, including tail rotor, engine and broadband. This method provides valuable insight into the periodic noise characteristics associated with the rotor, including amplitude, phase and directivity. $^{25}$

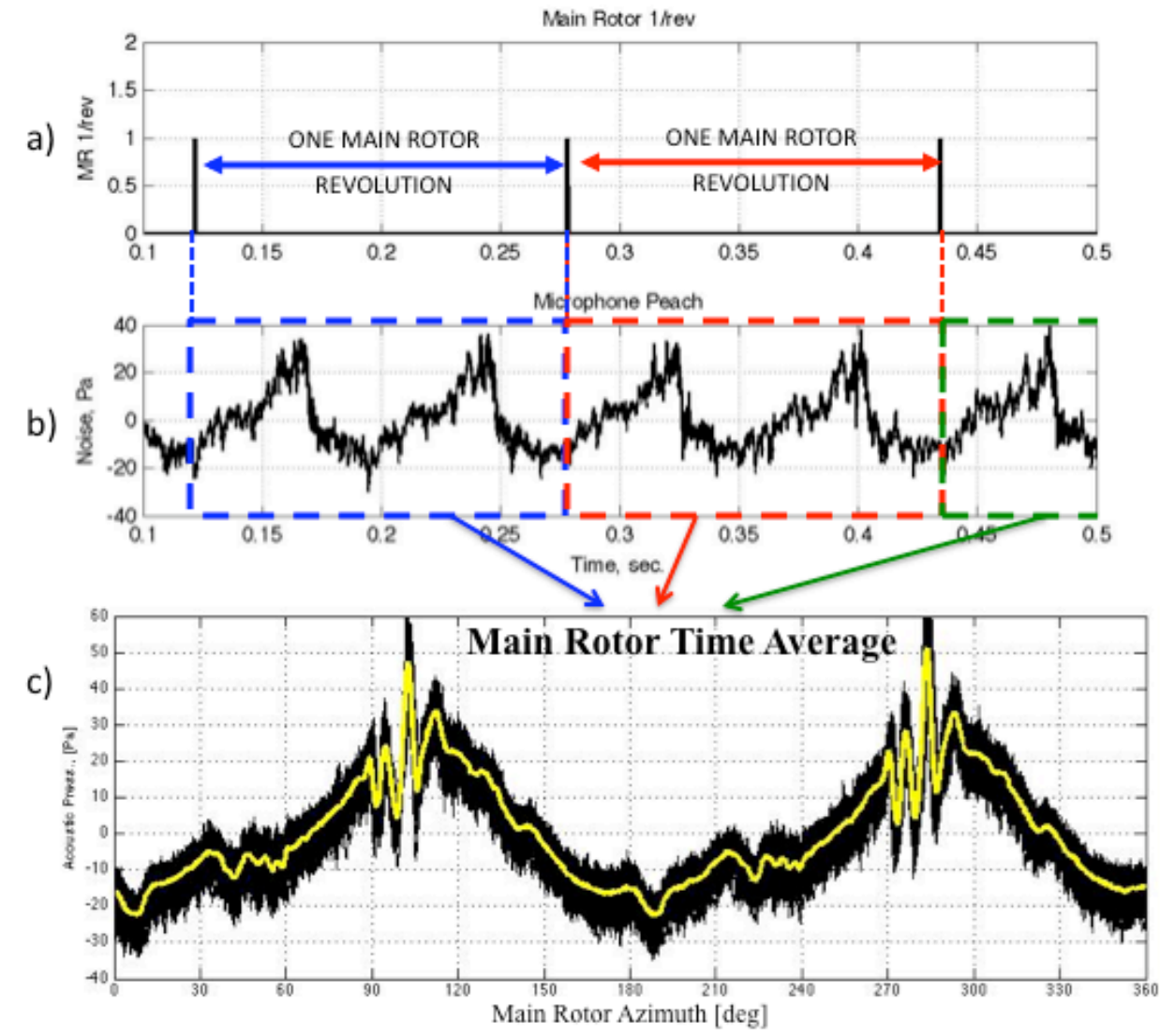

Figure 3.1: Main rotor time averaging for at $\mathbf{- 7 . 5}$ deg descent, $63.5 \mathrm{kts}$ 
The process of time averaging is relatively simple in nature. Measured sound pressures that occur during the span of a rotor revolution are interpolated to obtain acoustic samples that contain an equal number of data points (at equally spaced azimuth angle intervals). Then, any number of samples is averaged so as to drastically increase the signal-to-noise ratio of the harmonic source under investigation, with all other sources being averaged out.

An example of this averaging process is shown in Figure 3.1. This representative case is for the Bell 206B flying a $-7.5^{\circ}$ descent at $63.5 \mathrm{kts}$ over 64 consecutive main rotor revolutions recorded using microphone A3. In Figure 3.1 (c), the yellow line represents the time average, while the black lines represent the un-averaged acoustic samples that are being combined to form the average. This flight condition corresponds to a case in which we might expect a significant amount of BVI to occur. The major main rotor harmonic noise characteristics are seen, including the discrete impulsive character of the higher frequency BVI noise that occurs for this particular condition.

\subsection{Tail Rotor Time Averaging}

Figure 3.1 showed the merits of using a time domain averaging process to extract the harmonic noise of the main rotor from the overall acoustic measurement record of a helicopter in flight. The use of time domain averaging is also useful for the measurement of tail rotor noise. Because of the higher frequencies and general nature of tail rotor noise, many of the existing methods for noise measurement are less than ideal for the extraction of tail rotor time histories. Among these methods, ground based microphone 
arrays and wind tunnel tests account for the majority of the previous work that has been done (See Section 1.3).

\subsubsection{Drawbacks of Other Measurement Methods}

In addition to the reason described in Section 1.3, namely that the radiation vector between the source and observer changes as a function of time, many of the noise measurement techniques used in the past are also susceptible to other complications when attempting to examine tail rotor noise. While ground based microphone arrays and wind tunnel tests certainly provide good data in specialized areas, they lack the ability to provide the quality of measurement required for time domain averaging that the in-flight microphone array provides. Some of the reasons for this include:

1. The atmosphere more significantly attenuates tail rotor noise, which is at frequencies higher than the main rotor. This can result in uncertainty in the levels that is dependent upon the atmospheric modeling of the attenuation.

2. Doppler effects associated with the difference in speed between the source (the tail rotor) and microphones add an additional de-Dopplerization step to the process that can result in a significant amount of error when high-resolution knowledge of the helicopter orientation and location are unknown.

3. Reflected noise interference from walls or other sources complicates the averaging process if measurements from a wind tunnel or ground array are used. This is especially important for ground reflections during outdoors flight testing, when the impedance of the ground is often unknown.

4. Though a ground microphone array is capable of providing a considerable amount of information concerning the directivity of a rotor, the noise recorded at a 
particular microphone is at a different source time. It is then more likely to be occurring under a different flight condition, especially when examining transient maneuvers.

5. Scaling effects, particularly when concerning the interaction between the wake of the main rotor and the tail rotor, are for the most part unknown. Small models operating at scaled tip speed also operate at scaled Reynold's numbers that can alter the local aerodynamics of both the main and tail rotor.

Because the tail rotor is much smaller than the main rotor, tail rotor measurements can be made using externally mounted microphones and still be in the acoustic far field of the tail rotor, minimizing any near field effects that might interfere with the far field measurements. This fact alleviates the need for a leader aircraft. The dataset provided by the 2006 flight test and the accompanying University of Maryland Microphone Boom Array (see Chapter 2) is unique in that it provides high quality acoustic measurements in the far field of the tail rotor, moving at the same velocity as the helicopter. This negates many of the problems associated with the aforementioned measurement techniques.

\subsubsection{Methodology}

Though the dataset from the 2006 flight test clearly is useful for application to the study of tail rotor noise, two complications arose from using this data that had to be addressed. These included the lack of a tail rotor one-per-rev sensor and the need for a sample interpolation scheme.

As discussed previously, the existence of a main rotor one-per-rev sensor, and a knowledge of the net gear ratio between the main rotor and tail rotor of the Bell 206B 
made it possible to construct a theoretical tail rotor one-per-rev signal which was then used to average the acoustic pressures in the time domain. The first step in this process was to extract the digital main rotor one-per-rev peak (Figure 3.2 (b)) from the gradient of the raw analog signal obtained from the Hall-effect sensor (Figure 3.2 (a)). From this signal, the main rotor revolution period, as a function of time, could be computed, assuming that the rotor rotational frequency remained constant over the span of a single revolution (Figure 3.2 (c)). The main rotor revolution period was then used, in conjunction with the gear ratio of 6.472 , to calculate the tail rotor time period as a function of time (Figure $3.2(\mathrm{~d})$ ). The major assumption made during this process was that the main rotor revolution rate remained constant over any given main rotor revolution. This assumption was necessary as 6.472 tail rotor revolutions occur within a given main rotor revolution. In order to validate that the use of a constant main rotor rotational frequency was not a bad assumption, the standard deviation in the main rotor rotation frequency was calculated for each time averaging case sample (Table 3.1).

Table 3.1: Standard deviation in main rotor rotational frequency

\begin{tabular}{cccccc}
\hline \hline Case & Velocity [kts] & Flight Path & TR Rev. & MR Rot. Freq (Hz) & MR Std. Dev. \\
\hline 142 & 38 & 0 & 64 & 6.4777 & 0.002 \\
128 & 53 & 0 & 64 & 6.4402 & 0.0041 \\
136 & 62 & 0 & 64 & 6.4857 & 0.0033 \\
144 & 70 & 0 & 64 & 6.5121 & 0.0022 \\
211 & 63 & 0 & 64 & 6.3985 & 0.002 \\
212 & 63 & 3 & 64 & 6.4255 & 0.0011 \\
214 & 63 & 6 & 64 & 6.4367 & 0.0009 \\
215 & 63 & 7.5 & 64 & 6.4309 & 0.0015 \\
216 & 63 & 9 & 64 & 6.4387 & 0.0017 \\
219 & 63 & 12 & 64 & 6.4284 & 0.0009 \\
\hline \hline
\end{tabular}


From this table it is apparent that over the course of 64 theoretical tail rotor samples (the length of the averaged samples), the main rotor rotation frequency varied only slightly, with an average standard deviation of $0.00197 \mathrm{~Hz}$. Therefore, assuming a constant main rotor rotational frequency over a single revolution was an adequate estimation of the rotors' rotational frequency.

As a direct result of this assumption, the tail rotor rotational frequency was also considered to be constant over the duration of a single main rotor revolution. Tail rotor revolutions that overlapped between consecutive main rotor revolutions were assumed to be rotating at the revolution rate corresponding to the main rotor revolution in which the tail rotor revolution began (as indicated in Figure 3.2(d)). The tail rotor time period data was then used as a look-up table to generate an index of tail rotor $1 /$ rev occurrences that could be used for time averaging. The resultant tail rotor $1 /$ rev signal is shown in Figure 3.2(e).

a) Main Rotor $1 /$ rev Signal

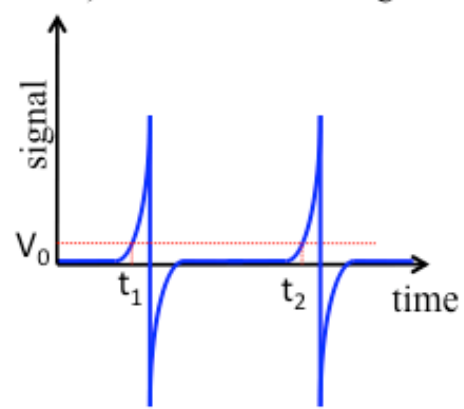

d) Tail Rotor Period

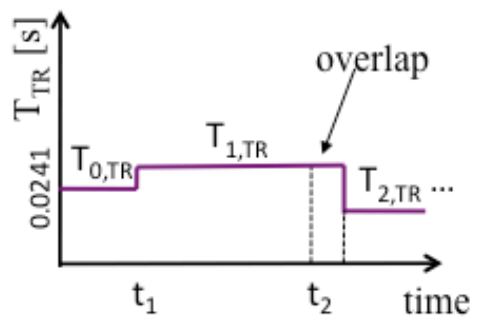

b) Filtered Main Rotor 1/rev Signal

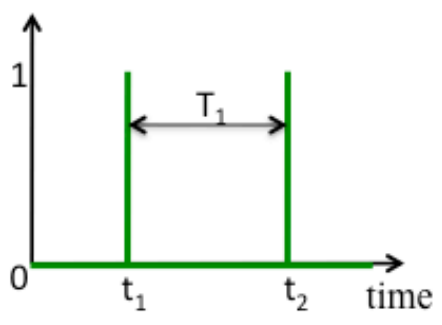

e) Tail Rotor $1 /$ rev Signal

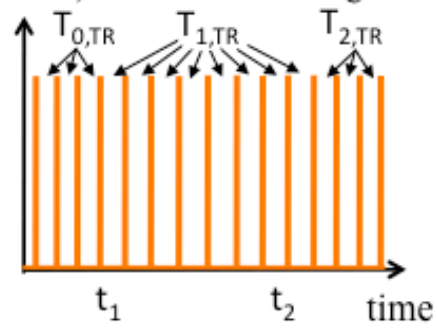

c) Main Rotor Period

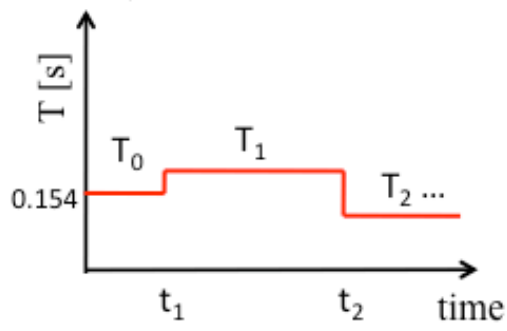

Figure 3.2: Generation of a theoretical tail rotor 1/rev signal from main rotor 1/rev signal 
The next step in the time averaging process involved the manipulation of acoustic data into arrays of equal length that could then be added and averaged. This was achieved using the tail rotor $1 / \mathrm{rev}$ index array. This array, which contained the locations within the acoustic data file at which the tail rotor completed a revolution, was used to split the acoustic measurement file into smaller data files, each corresponding to a specific tail rotor revolution. The process was started at the first available main rotor $1 / \mathrm{rev}$ signal in the dataset, as shown in Figure 3.3 (b)). Because of the variation in main, and thus tail rotor, rpm, these acoustic samples were of slightly varying length and could not be simply added. A linear interpolation scheme (provided by MATLAB) was used to reduce all data samples to arrays containing the number of data points that the smallest sample contained (approximately 480 points in most cases). Each acoustic file then contained data corresponding to the appropriate azimuth angle of the tail rotor and could be properly averaged.

It is important to note that while this data could be averaged within a given global data set (corresponding to a given flight), data from different flight-tests could not be properly combined. This is because of the method with which the theoretical tail rotor $1 /$ rev was generated (starting at the first main rotor signal). Without a full knowledge of the starting position of the tail and main rotors, the phasing between the two data sets is completely unknown. It is also significant to note that datasets for tail rotor revolutions can theoretically be averaged for non-consecutive revolutions. However, to minimize the consequences of changes in flight condition, the data is chosen such that a relatively constant flight condition is maintained throughout the dataset. 
Previous work that has taken in-flight measurements of helicopter noise and used time averaging techniques, have found that the number of revolutions used in the averaging process can significantly affect amplitude of the time averages. ${ }^{27}$ By increasing the number of revolutions over which the data is averaged, large reductions in any contributions from broadband noise and other noise sources not associated with the tail rotor are gained. However, doing this also increases the potential for flight condition unsteadiness, which could reduce the amplitude of the contribution from the tail rotor itself. As will become evident in the coming chapters, the tail rotor noise is dominated heavily by simple thickness and loading noise, phenomena which are not particularly susceptible to small changes in flight condition. It was found therefore that the peak amplitudes changed very little when using 8,32, 64, 128 or 256 (powers of two were used to speed processing). Therefore a value of 64 tail rotor revolutions (or approximately 1.54 seconds) was chosen for the time averaging process as it provided both good time averages and expedited the averaging process.

An example of the tail rotor time averaging process is shown in Figure 3.4 for the same flight condition as was shown for the main rotor (Figure 3.1). At $-7.5^{\circ}$ descent, $63.5 \mathrm{kt}$ flight, the time averaging process is successful in isolating the pulse shape of the tail rotor harmonic noise, even in the presence of a considerable amount of main rotor BVI noise. This is important as main rotor BVI occurs in roughly the same frequency range as the tail rotor operates. In Figure 3.4 (c), one can clearly identify the characteristic thickness noise pulses as the tail rotor progresses through one complete rotor revolution. Nonperiodic noise has been removed from the time averaged acoustic pulse. Note also that the un-averaged acoustic pulses, shown in black in Figure 3.4 (c), show considerable 
scatter about the time average signal. This scatter, which is considerably larger than that seen in the main rotor time average, is due to the large levels of main rotor harmonic noise that are averaged out by the tail rotor averaging process. In subsequent timeaverages displayed throughout this report, an $80 \mathrm{~Hz}$ high-pass band filter has been applied to these un-averaged pulses after the averaging process, purely for visualization purposes.

a)

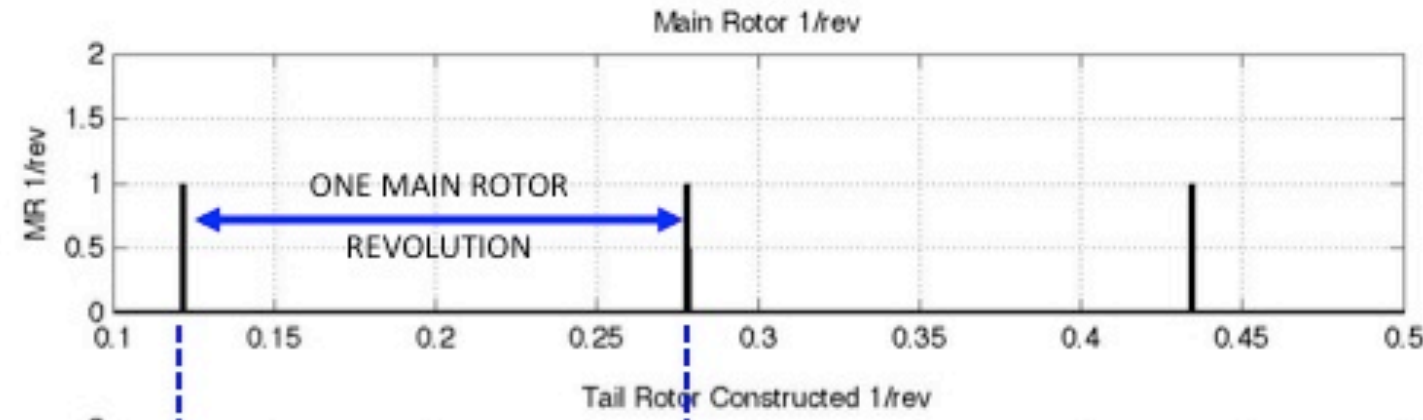

b)

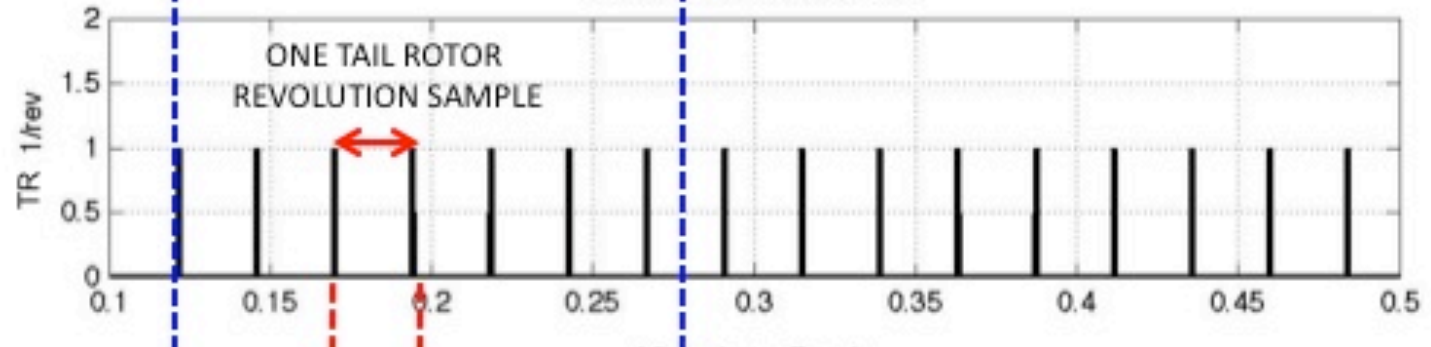

c)

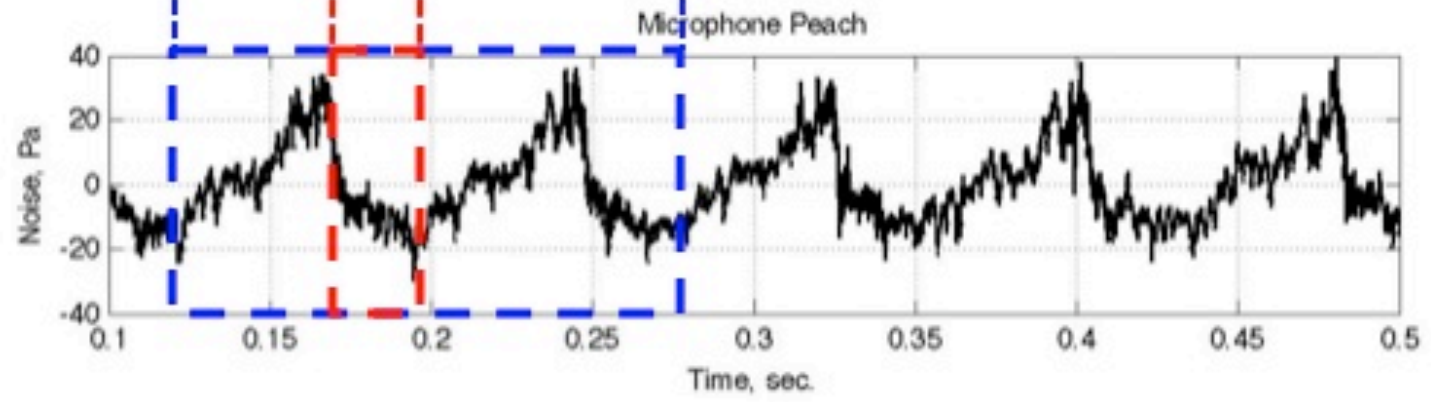

Figure 3.3: Acoustic data reduction technique for time averaging 


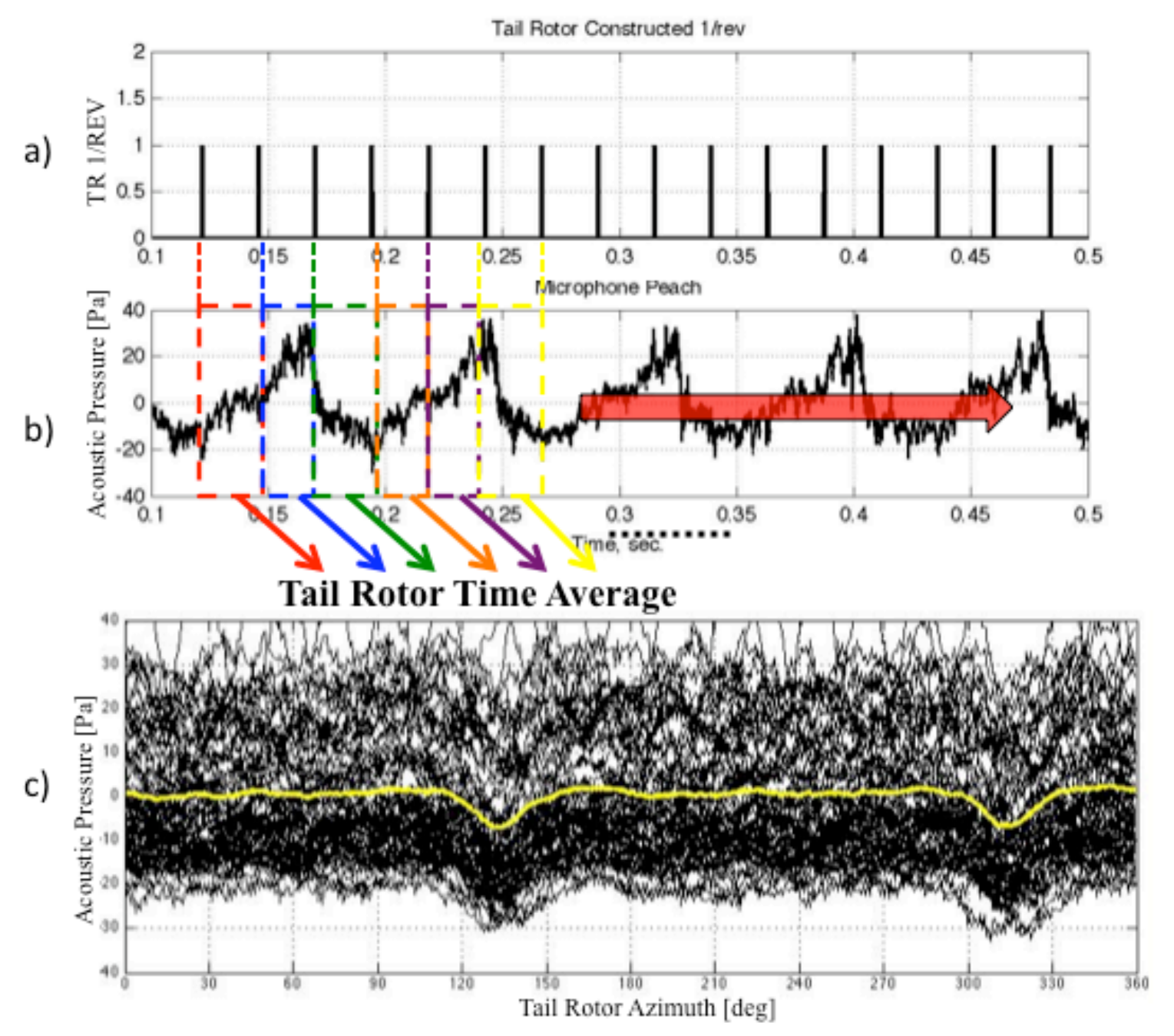

Figure 3.4: Tail rotor time averaging schematic for Bell $206 \mathrm{~B}$ at $-7.5 \mathrm{deg}$ descent, $63.5 \mathrm{kts}$

\subsection{Discrete Fourier Transformation (DFT) Analysis}

In addition to a time domain analysis, frequency domain analysis, using Discrete Fourier Transformation (DFT), can also be used to examine an averaged Sound Pressure Level (SPL) spectrum of acoustic data. For this analysis, a comparison between the spectral average (RMS average of 32 DFT's of two-second, consecutive (but overlapping) samples of data for the 206B helicopter) and the DFT of both the main rotor and tail rotor time averages (shown in Figure 3.1 and Figure 3.4) can be made. 
The spectral average was computed using the following equations,

$$
\begin{aligned}
& P_{R M S}=\sqrt{\frac{1}{n} \sum_{i=1}^{n}\left(\left|P_{i}\right|^{2}\right)} \\
& \text { Spectral Average }[d B]=\left|20 \log _{10}\left(\frac{P_{R M S}}{P_{R E F}}\right)\right|
\end{aligned}
$$

Where $\mathrm{P}_{\mathrm{i}}$ represents the array containing the FFT of a two-second sample of acoustic pressure data, $\mathrm{n}$ is the number of samples used $(\mathrm{n}=32)$, and the reference pressure used for conversion to sound pressure level, $\mathrm{P}_{\mathrm{REF}}=2 \times 10^{-5} \mathrm{~Pa}$.

In Figure 3.5 (a), which corresponds to the $-7.5^{\circ}$ descent case at $63.5 \mathrm{kts}$, both the main rotor and tail rotor harmonics are shown that are distinct multiples of the main and tail blade passing frequency. The dominance of main rotor harmonic noise at high frequencies is indicative of a flight condition with a large degree of BVI noise. It is important to note that while this method does provide valuable information regarding the frequency content and energy associated with the main and tail rotor harmonic noise, it does not capture phase information. This information is crucial for a full understanding of the mechanisms that are associated with a particular noise source.

A similar analysis for the helicopter flying at 63.5 knots, steady, level flight is shown in Figure 3.5 (b). An additional benefit of the SPL spectrum is that is provides insight into the importance of different noise sources at certain frequencies and flight conditions. In Figure 3.5 (a), the harmonic noise at multiples of the main rotor blade passage frequencies in descent is significantly larger when compared to the steady level flight case, Figure 3.5 (b). This indicates that the tail rotor is really of secondary importance in this descending flight condition. However, in Figure 3.5 (b), it is clear that the tail rotor harmonic noise levels are significantly larger than those shown in Figure 3.5 (a), and that 
the amplitudes far exceed any main rotor harmonic noise in the same frequency range. This is indicative of the fact that more tail rotor thrust, which results in more tail rotor harmonic noise, is required to trim the helicopter in steady level flight. An analysis of this nature is perhaps the most effective way of showing the importance of tail rotor noise in this 100 to $1000 \mathrm{~Hz}$ frequency range, which is of particular concern for human annoyance. However, it leaves a lot to be desired in terms of an understanding of what exactly is responsible for generating the noise itself.

Beyond the information that a frequency domain analysis can provide, the spectral averages can also be used to compare the time and frequency averaging processes. This comparison is also shown in Figure 3.5 (a) and (b). In addition to the spectral averages, a DFT was taken for the time-averaged acoustic data for both the main and tail rotors (see for example, Figure 3.1 and Figure 3.4) for the descending flight condition at $-7.5^{\circ}$ at 63.5 knots (Figure 3.5 (a)) and steady level flight (Figure 3.5 (b)). The peak values of the DFT of the time averaged main rotor harmonic noise are shown as blue circles, while the DFT of the time averaged tail rotor harmonic noise are shown as red diamonds. These markers represent the average harmonic power that is being radiated by the main and tail rotor, and that is being captured by the time averaging process. The bandwidth of the DFT of the averaged time histories is $6 \mathrm{~Hz}$ and $40 \mathrm{~Hz}$ respectively, which was dictated by the sampling rate of $20 \mathrm{kHz}$. Very close agreement between the two methods is demonstrated up to fairly high numbers of harmonics for both the main and tail rotor indicating that either method can be used to determine the average noise power being radiated by either rotor. There is also a slight under prediction in SPL at higher frequencies due to small changes in main rotor rotational frequency. 

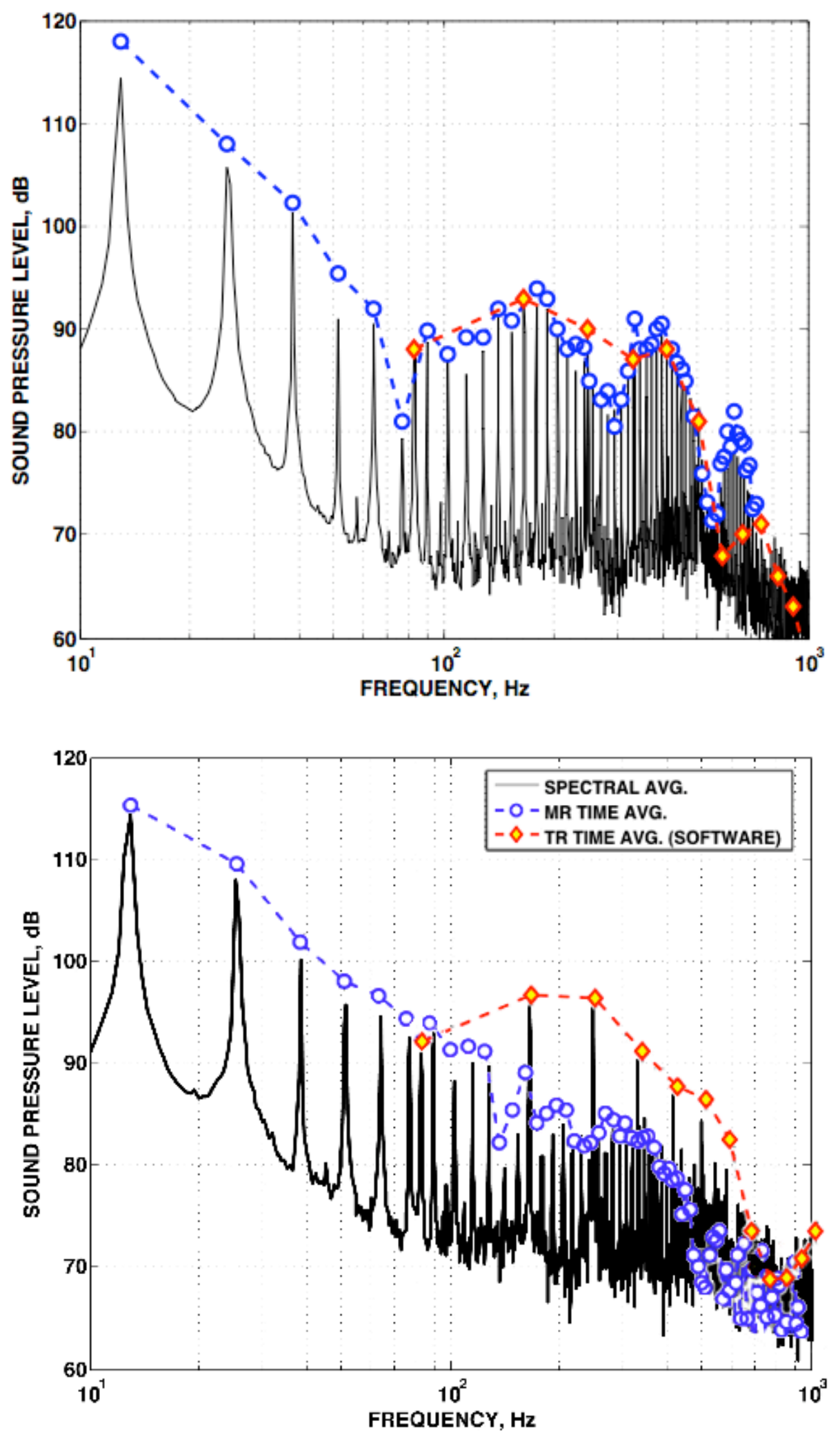

Figure 3.5 : SPL spectra for A2 at $63.5 \mathrm{kts}$ a) -7.5 deg descent b) level flight 


\section{Chapter 4 Experimental Results}

\subsection{Summary}

This thesis focuses on the data taken during the 2006 flight test for both steady level flight and steady descending flight. Because of the increased drag and weight associated with the microphone boom array, the Bell 206B was limited in its maximum forward velocity. As a result, forward flight data was taken at speeds ranging from approximately 40 knots to 75 knots. Steady descent data was recorded at a speed of approximately 63 knots, with descent angles ranging from $0^{\circ}$ to $-12^{\circ}$. Table 4.1 summarizes the cases that were used for this research.

Table 4.1: Summary of flight conditions

\begin{tabular}{ccc}
\hline \hline Case (Data Set) & Velocity [knots] & Descent Angle [degrees] \\
\hline Case 1 (142) & 38 & 0 \\
Case 2 (128) & 53 & 0 \\
Case 3 (136) & 62 & 0 \\
Case 4 (144) & 70 & 0 \\
Case 5 (211) & 63 & 0 \\
Case 6 (212) & 63 & -3 \\
Case 7 (214) & 63 & -6 \\
Case 8 (215) & 63 & -7.5 \\
Case 9 (216) & 63 & -9 \\
Case 10 (219) & 63 & -12 \\
\hline \hline
\end{tabular}

\subsection{Data Portrayal}

All of the acoustic time averages in this chapter are presented in the same fashion. The abscissa of each plot represents the tail rotor azimuth angle in units of degrees. The ordinate of each plot represents the normalized acoustic pressure, in Pascals, of the given 
time history. The yellow line in each plot is the acoustic time average obtained using the averaging process described in Chapter 3. Each time average has been scaled to a distance of $10 \mathrm{R}_{\mathrm{TR}}(26 \mathrm{ft})$ from the tail rotor hub so that directivity information can be better understood. The signals represented in black are the un-averaged revolution samples with an $80 \mathrm{~Hz}$ High-Pass Band Filter applied after the averaging process has been completed (the averaging process is applied to unfiltered acoustic data). This unaveraged data is included as it provides a quick visual check for data consistency and quality. Finally, each plot displays the calculated overall sound pressure level (OASPL) for that particular time average of tail rotor noise. These OASPL values are then summarized in figures at the end of each flight condition section. The plots shown in this chapter were chosen so at to efficiently convey the information necessary for an understanding of the conclusions that are drawn.

\subsection{Level Forward Flight}

The examination of the time averaged data for microphone A1 (See Figure 2.7), the microphone closest to the tip-path-plane of the tail rotor, is shown in Figure 4.1 for level flight at a variety of increasing forward velocities. The microphone is located approximately $16.8^{\circ}$ above (toward the starboard side of the helicopter) and is the closest

microphone to the tail rotor. Figure 4.1 (a) through (d) clearly show that two primarily thickness noise peaks dominate the time average histories during the time of one complete tail rotor revolution. This is logical given that thickness noise is radiated mostly in the plane of the rotor, whereas any influence of loading noise is reduced near the plane of the rotor. The peak-to-peak values, as well as the OASPL, also increase slightly $(\sim 3$ dB) as the forward velocity of the helicopter increases from 38 to 70 knots. 


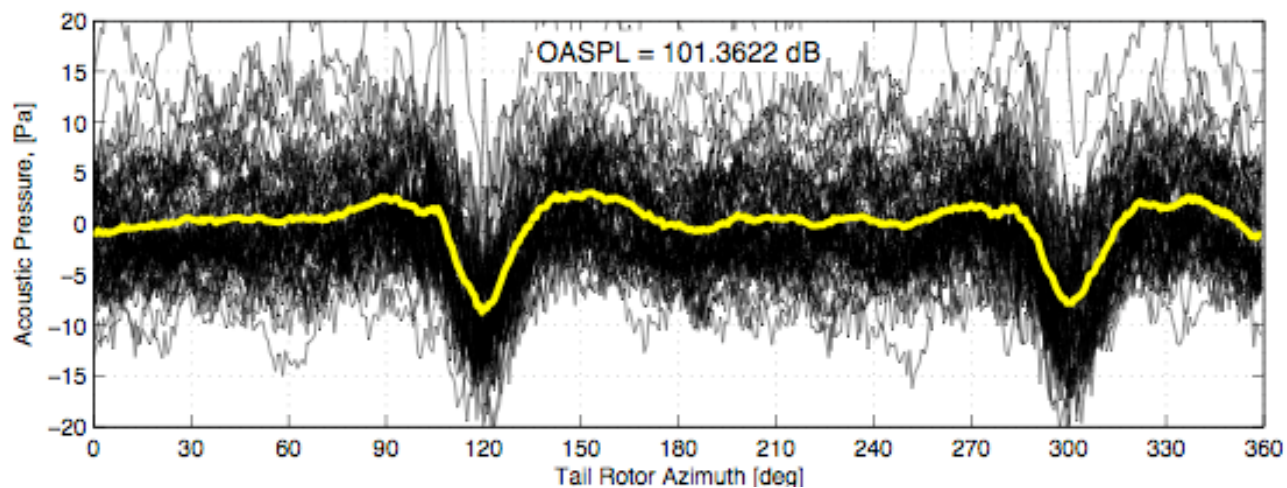

(a): $A 1(38 \mathrm{kts})$

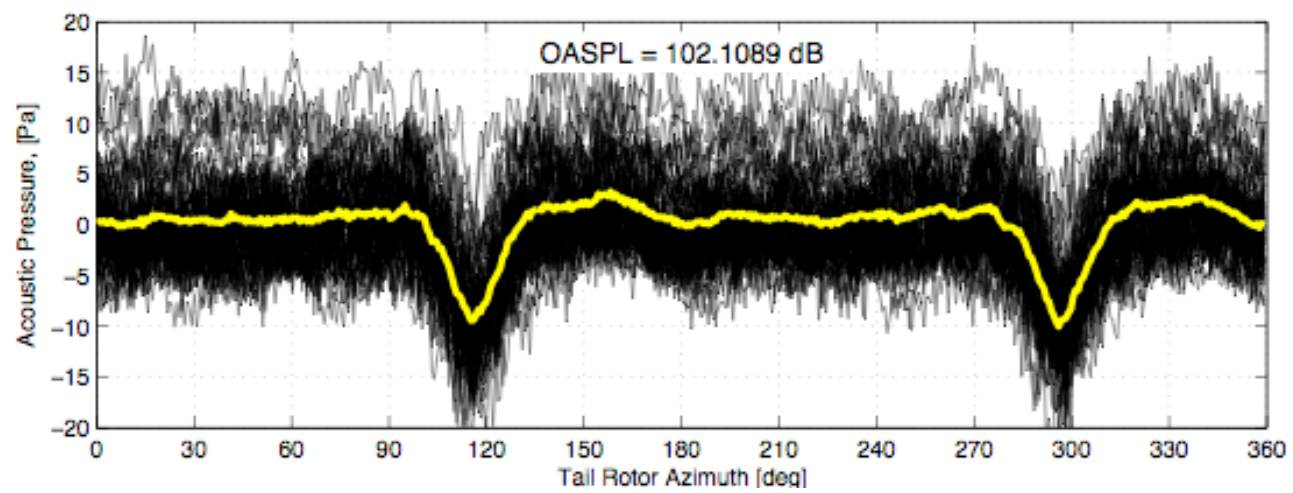

(b): A1 (53 kts)

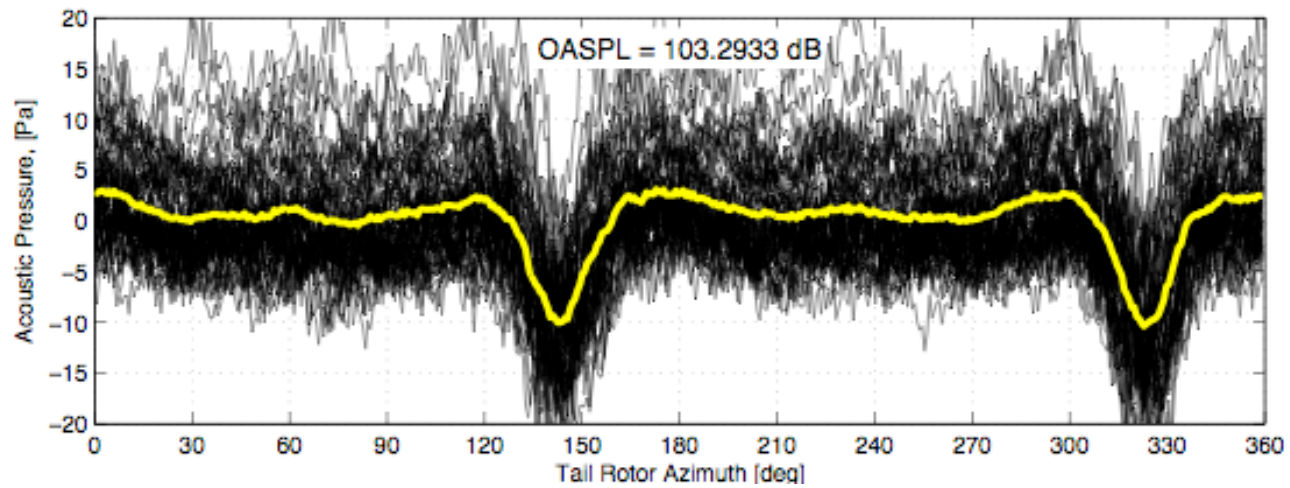

(c): $A 1$ (62 kts)

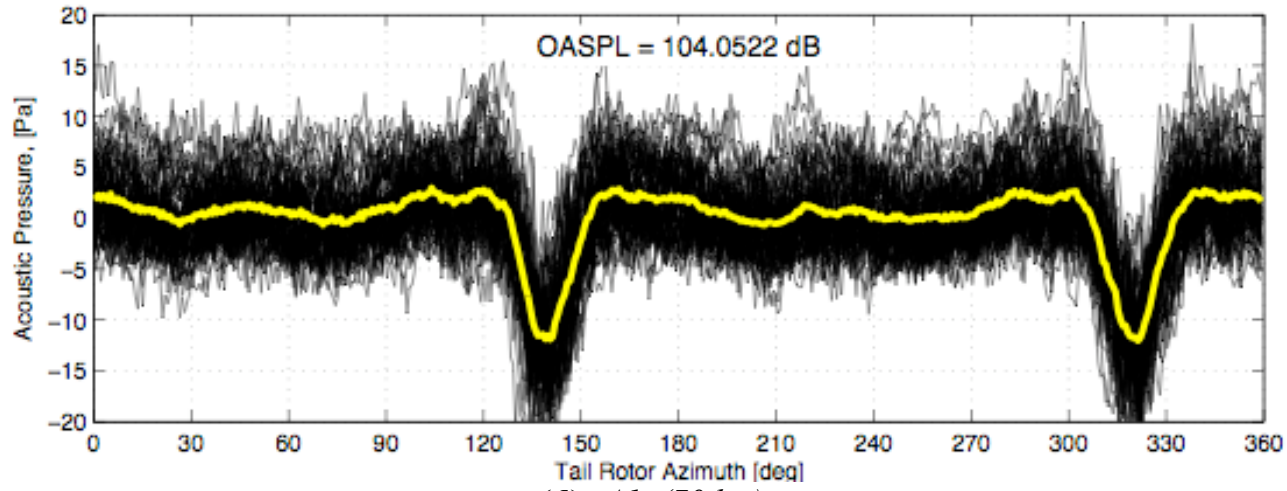

(d): $A 1$ (70 kts)

Figure 4.1: Acoustic time average at microphone A1 as a function of forward velocity 
In addition to providing information about a single observer location, the time averaging technique also provides valuable information concerning the directivity of the tail rotor noise. Figure 4.2 (a) through (d) allow for a comparison of the data recorded at all microphone positions for the 62 knot flight condition. Thickness noise appears to dominate near the plane of the rotor $(\mathrm{A} 1 \& \mathrm{R} 1)$, while loading noise dominates the time histories at the outer microphone positions (A4 \& R2). OASPL also increases as the inner microphones are approached. Though it is likely that the noise would reach a maximum directly in the plane of the rotor, no microphone was located there. Interference and direct reflections from the helicopter fuselage discouraged the placement of a microphone at this position.

Another interesting feature of Figure 4.2 is that the averages obtained for the starboard microphones (A1, A2, A3, and A4) display very good blade-to-blade consistency. In other words, peak amplitudes and pulse width remain relatively constant from blade to blade within a particular time average history. On the opposite side of the helicopter fuselage (R1 and R2), where superior signal quality might be expected due to the clear path between the source and the observer, the time averages show far less clear results. Though microphones A2 and R1, as well as A4 and R2, are nearly equidistant from the tail rotor hub, the port side microphones appear to show a substantial decrease in signal quality and level. This is partially due to the linear summation of the mechanisms associated with thickness and loading noise. This is associated with the respective peak shape and phasing of the loading and thickness noise pulses. 


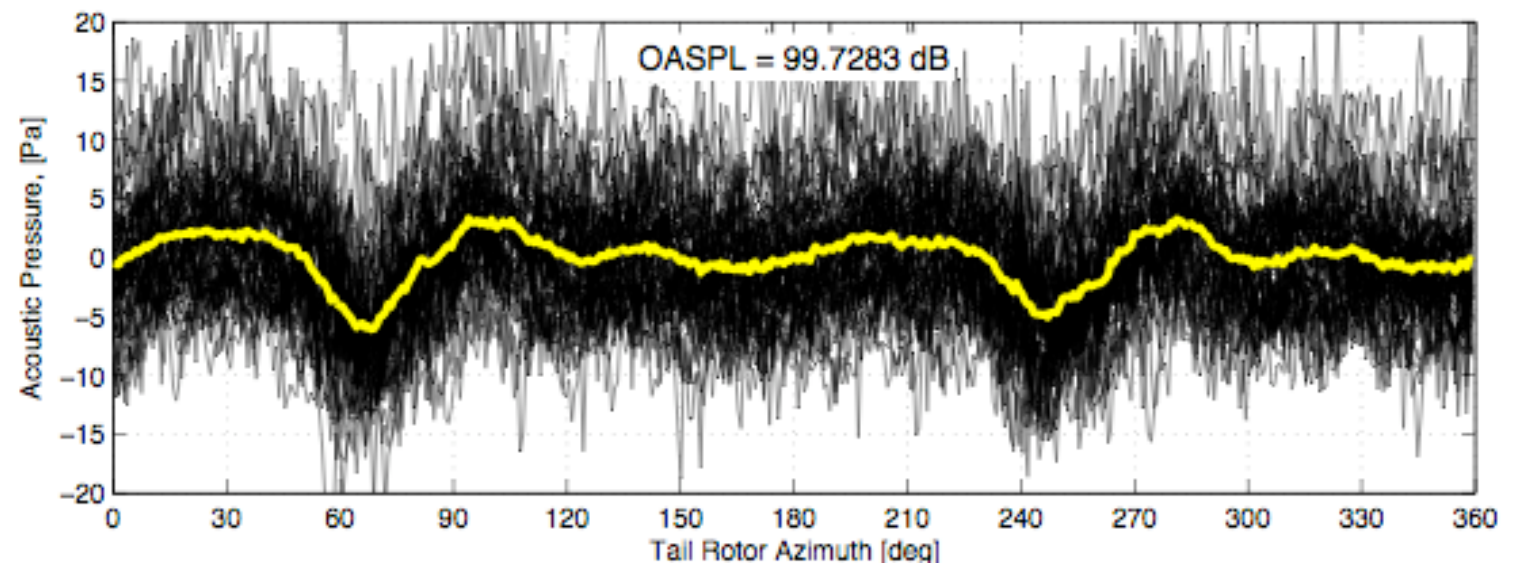

(a): $A 4(62 \mathrm{kts})$

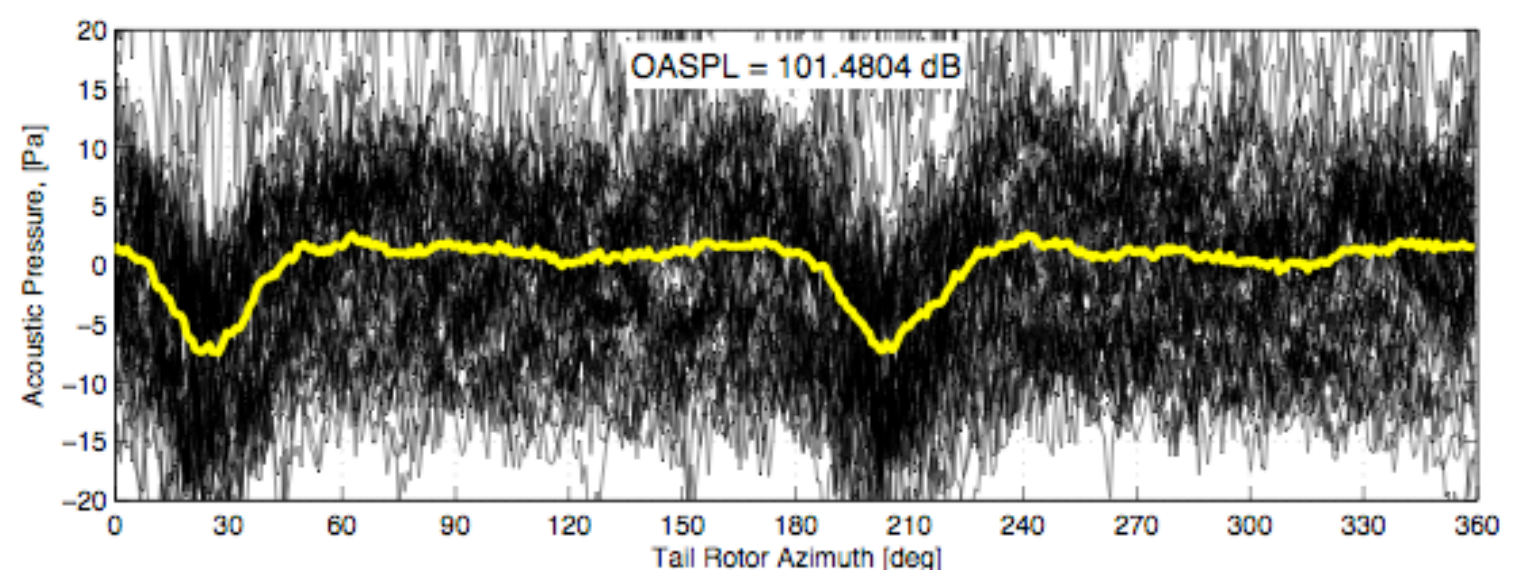

(b): $A 3(62 \mathrm{kts})$

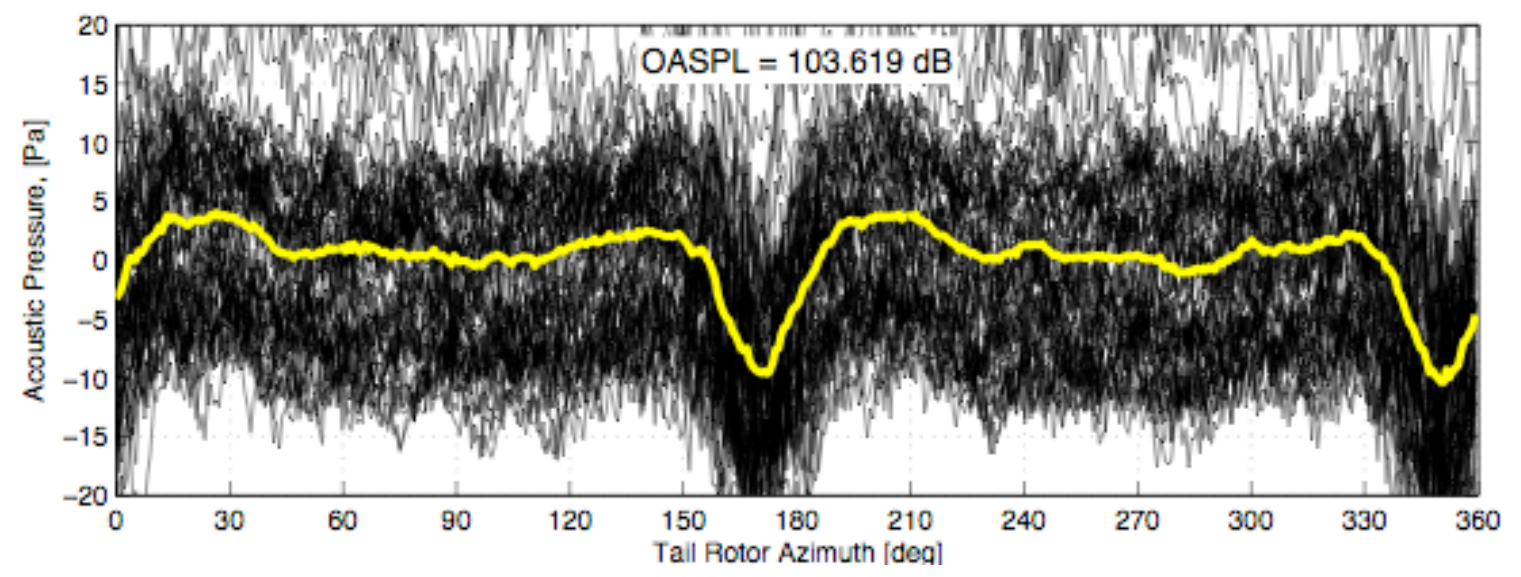

(c): $A 2(62 \mathrm{kts})$ 


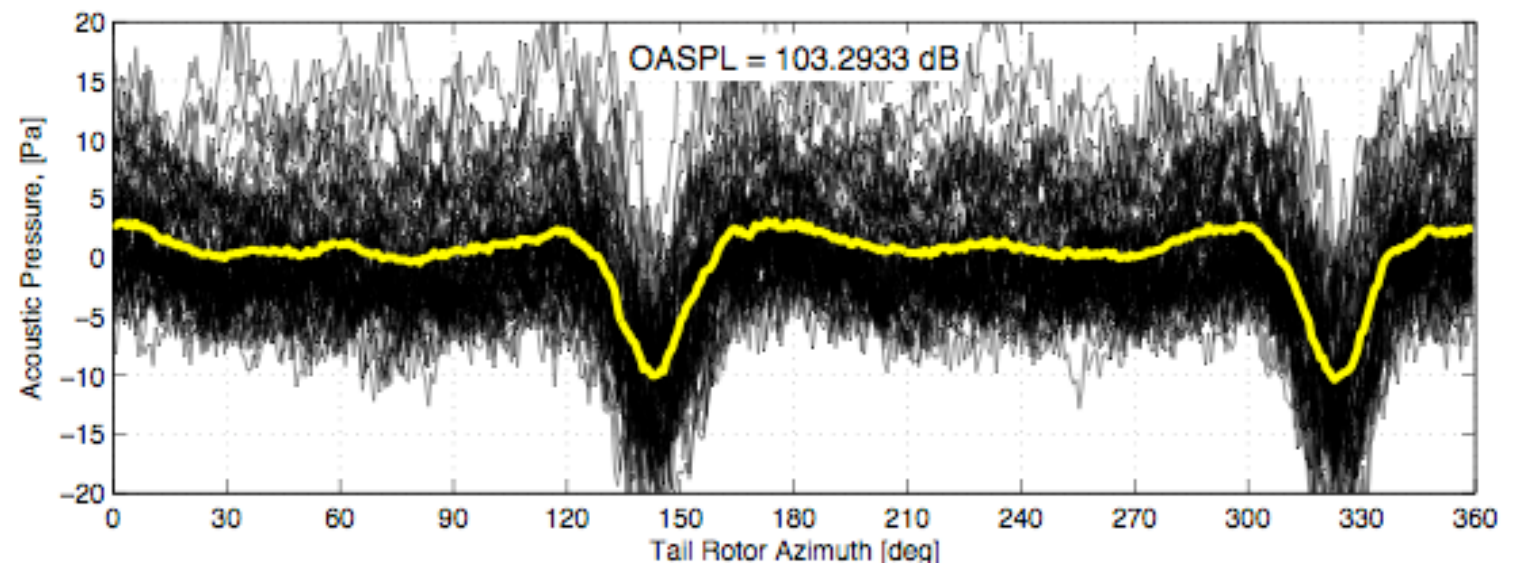

(d): $A 1(62 \mathrm{kts})$

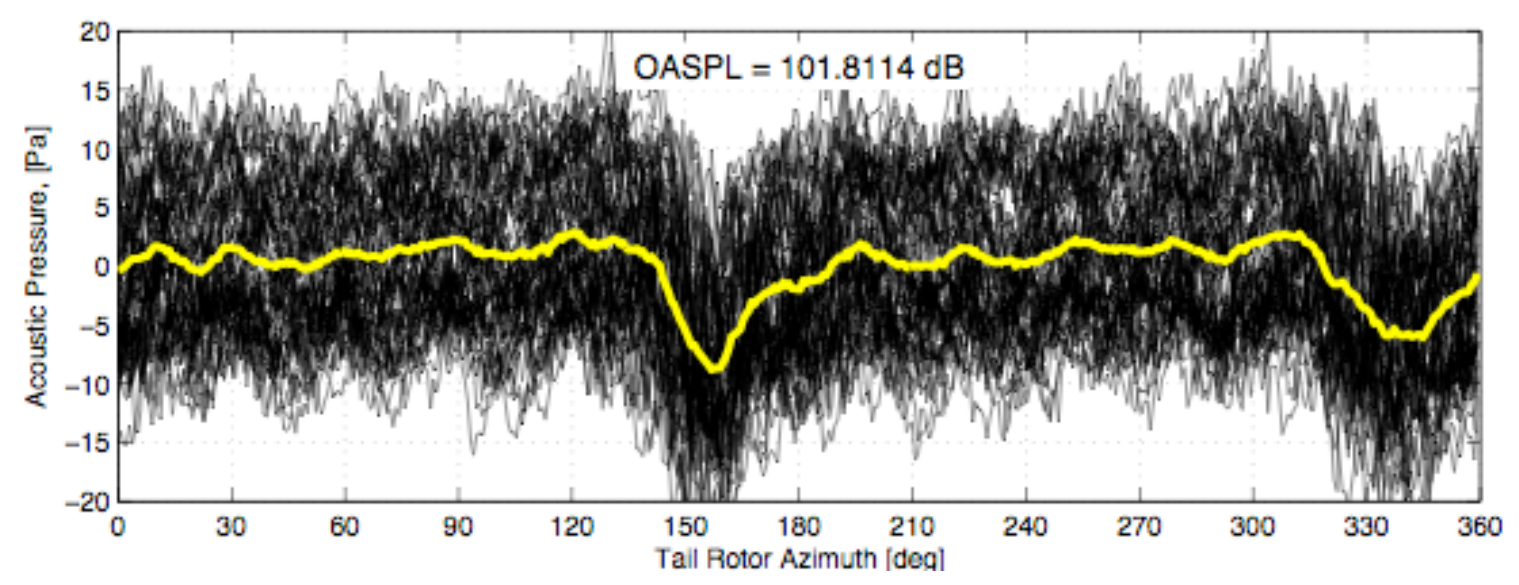

(e): $R 1(62 \mathrm{kts})$

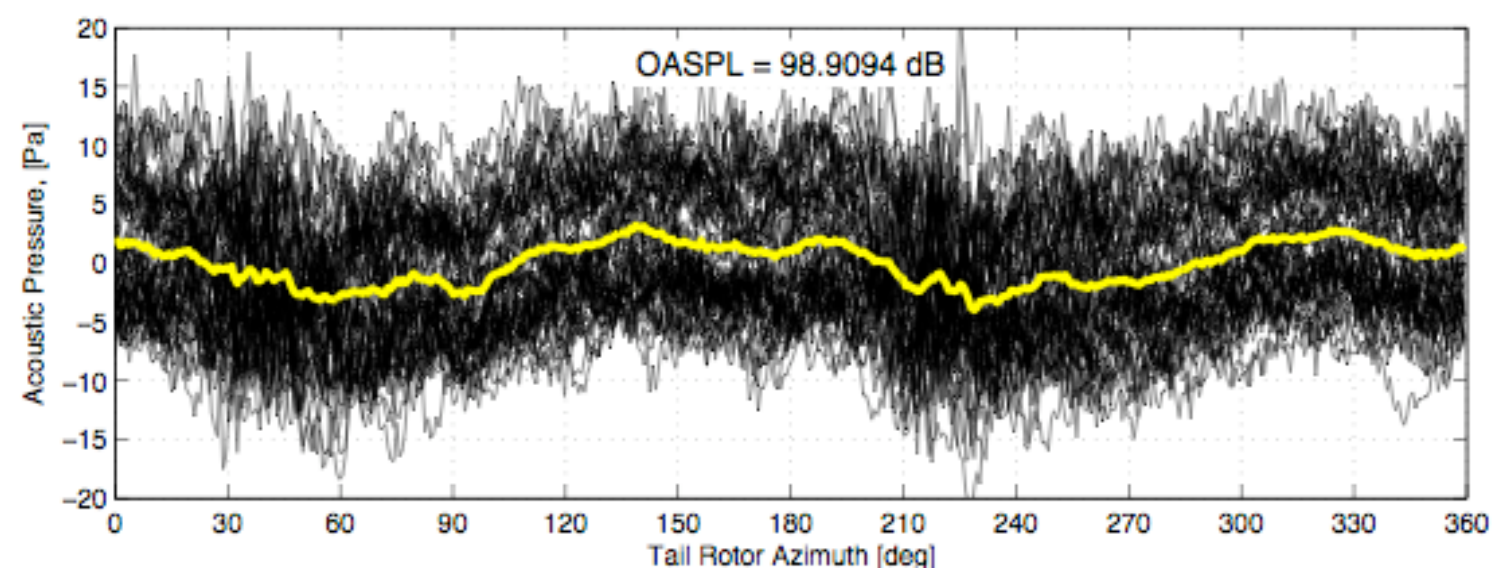

(f): $R 2(62 \mathrm{kts})$

Figure 4.2: Directivity pattern of tail rotor noise at 62 knots 
Generally, loading noise radiated towards the starboard side of the helicopter reinforces the negative thickness pulse to amplify noise. The port side microphones instead experience a reduction in total noise level due to the partial cancellation between loading and thickness noise.

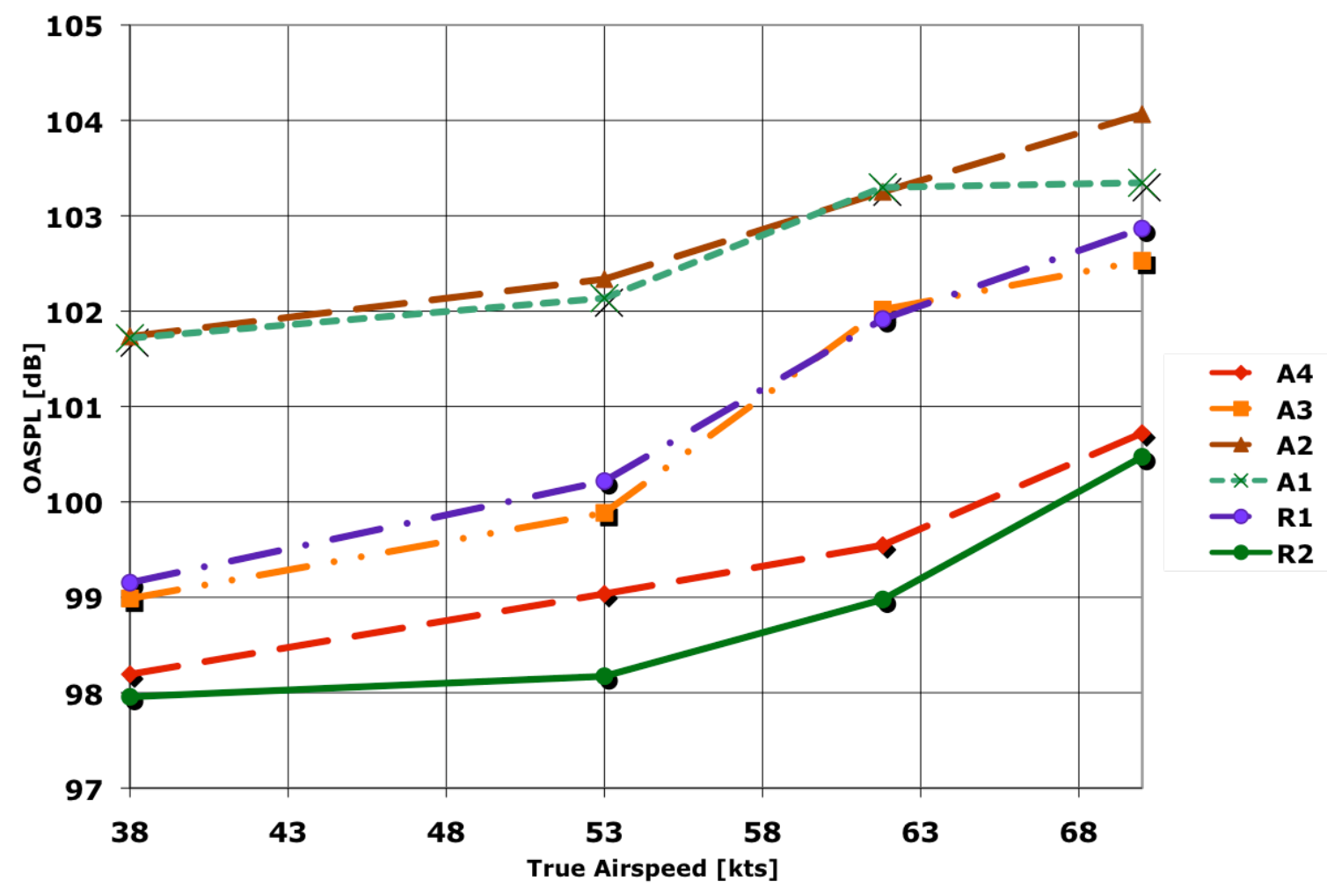

Figure 4.3: Variation in OASPL as a function of airspeed for all microphones

Beyond looking at individual time histories, the data set obtained in 2006 provides the ability to examine the acoustic trends as a function of flight condition. The change in OASPL as a function of forward velocity for all microphone positions is shown in Figure 4.3. There is a general increase in OASPL of approximately $3 \mathrm{~dB}$ for the Bell $206 \mathrm{~B}$ as forward velocity is increased from 38 to 70 knots. The OASPL is also approximately $4 \mathrm{~dB}$ lower for the out-of-plane microphones as it is for the near in-plane microphones. Figure 
4.3 indicates that tail rotor noise is loudest directly in the plane of the tail rotor during forward flight for the 206B helicopter. As seen in Figure 4.1, thickness noise is the dominant source of noise at these near in-plane microphone positions.

\subsection{Steady Descending Flight}

In addition to level flight, the data set generated during the 2006 flight test provided for the opportunity to examine the behavior of the tail rotor noise during steady descending flight. Table 4.1 (page 56) lists the cases that were studied for descending flight. In order to decouple the effects of change in forward velocity and descent angle, the cases looked at were chosen carefully so as to maintain a true airspeed of $63 \pm 0.2$ knots. Figure 4.4 (a) through (f) show the results for microphone A1 as a function of descent angle.

It is apparent that the noise decreases approximately $2 \mathrm{~dB}$ for this near in-plane microphone as the steady descent angle progresses from $0^{\circ}$ to $-12^{\circ}$. The time histories in Figure 4.4 also still appear to be dominated by the thickness noise type pulse that was seen in the level flight condition (Figure 4.1). If however we look at an out-of-plane microphone, we might expect the decrease in OASPL to be slightly larger. This is because as we increase our descent angle, the main rotor torque that must be counteracted by the tail rotor is being decreased. As explored in Chapter 5, this results in a reduction in the net loading that the tail rotor must provide, and thus, a lower level of loading noise. Loading noise, as shown in Figure 4.2 for level flight, is the dominant noise mechanism at the out-of-plane microphone positions. 


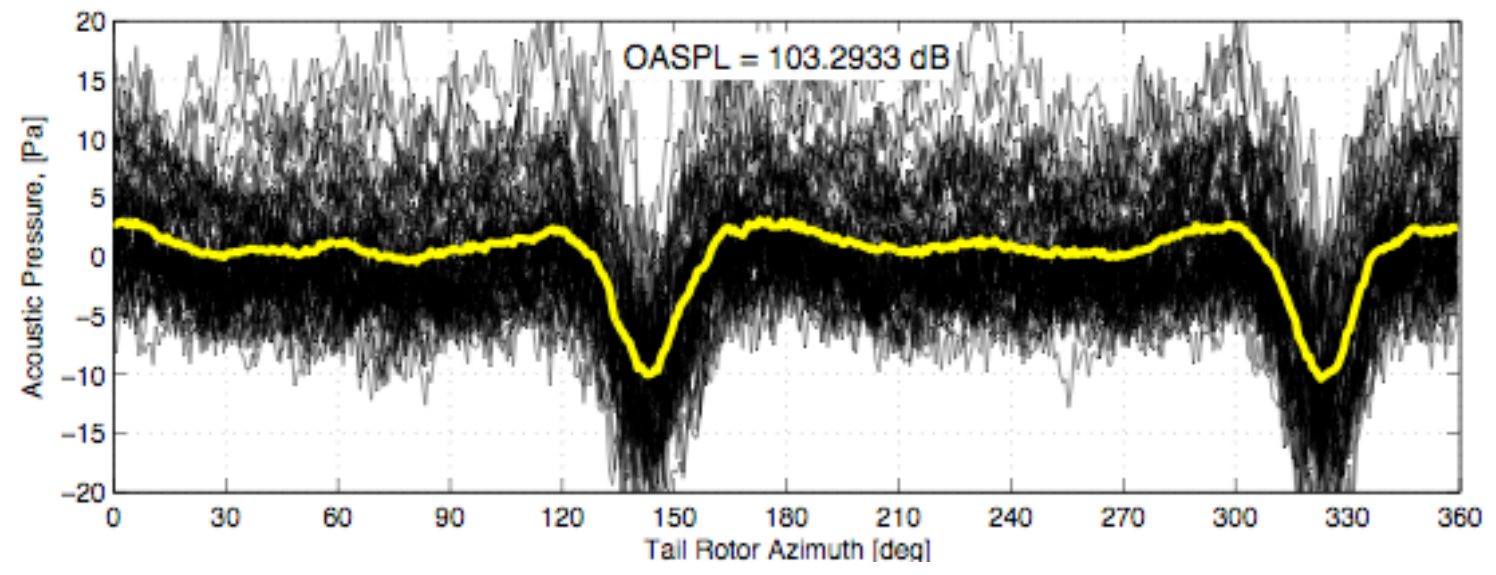

(a): $A 1$ (0 degrees)

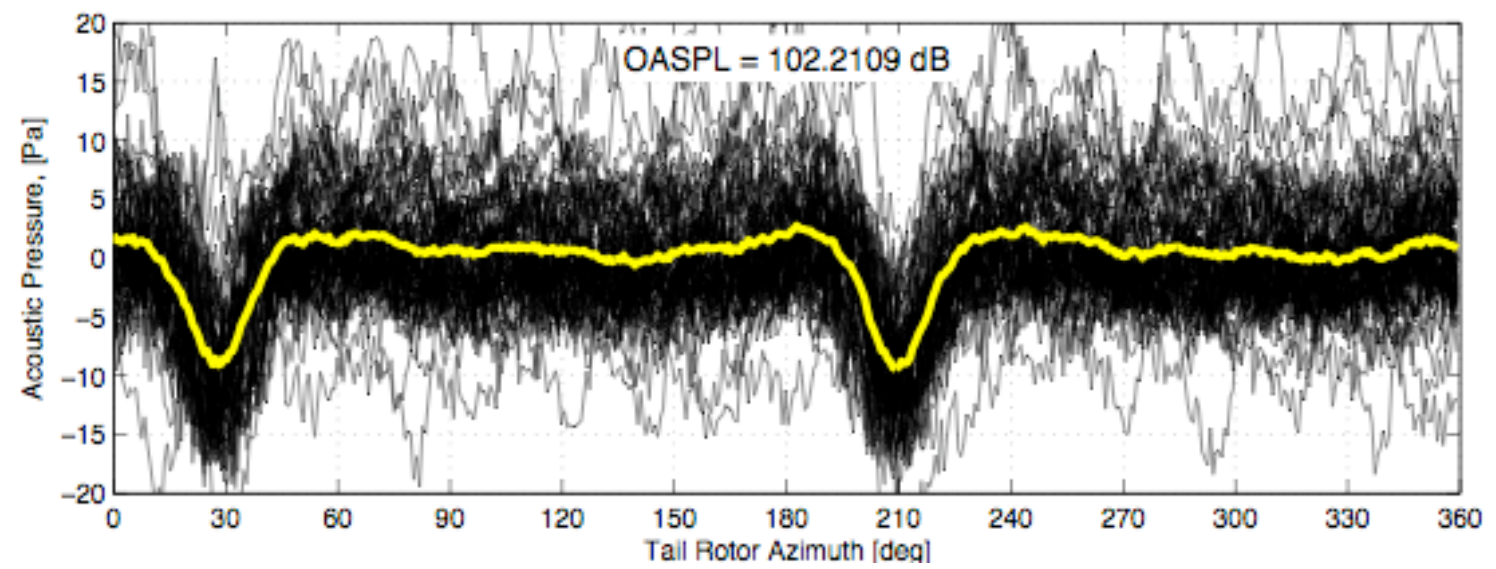

(b): A1 (- 3 degrees)

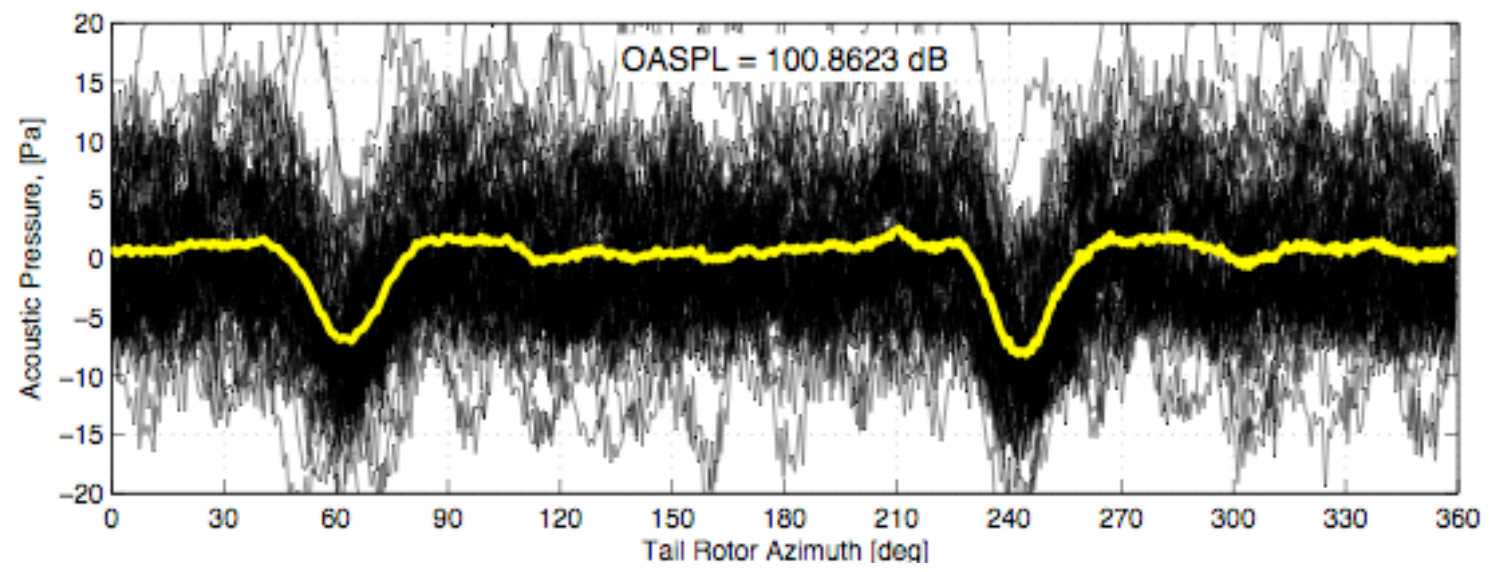

(c): A1 (- 6 degrees) 


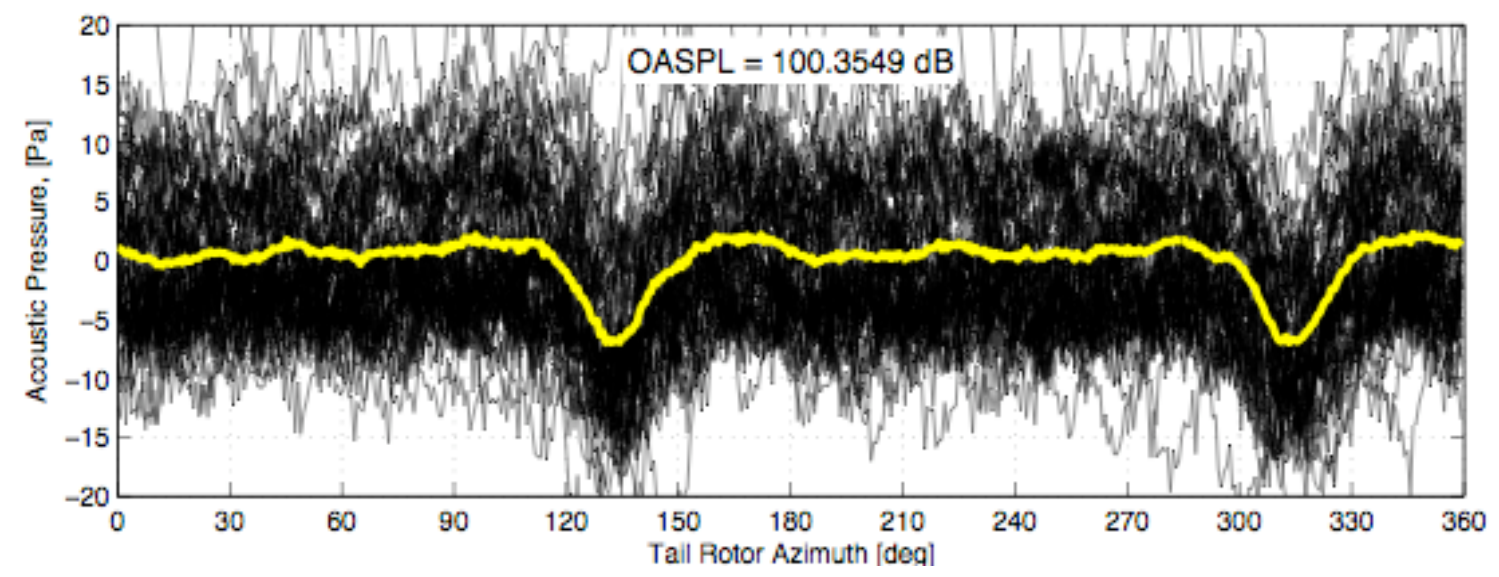

(d): $A 1$ (- 7.5 degrees)

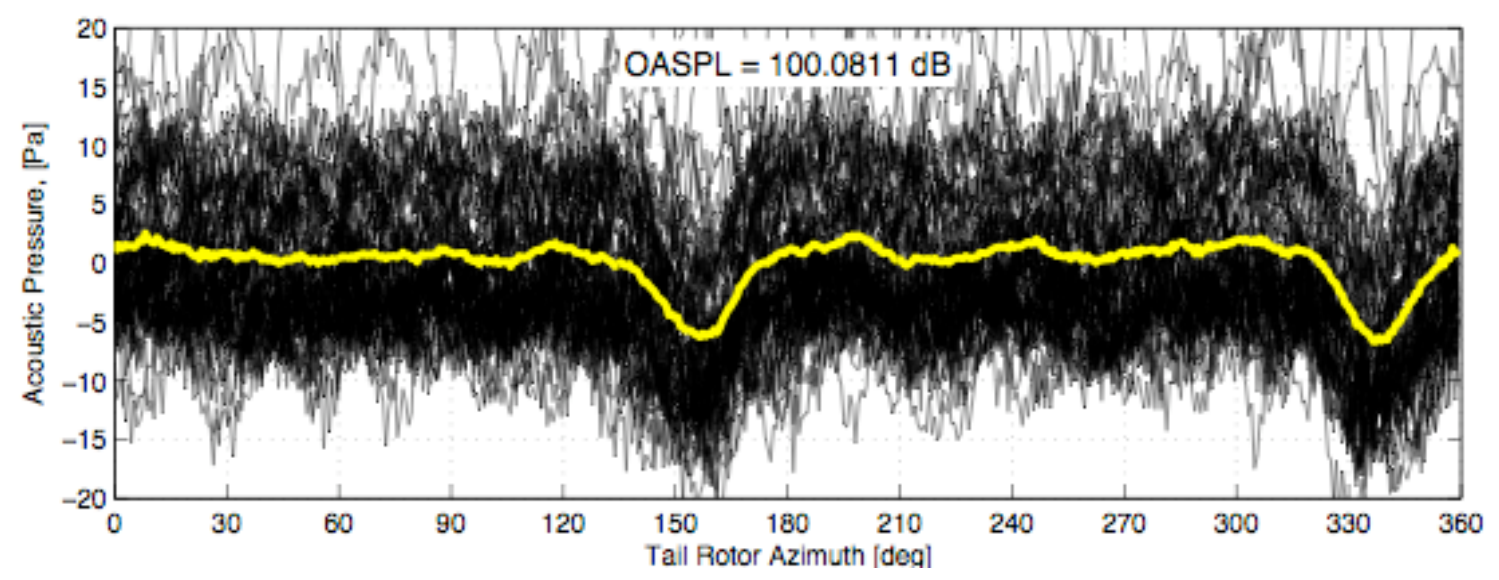

(e): A1 (- 9 degrees)

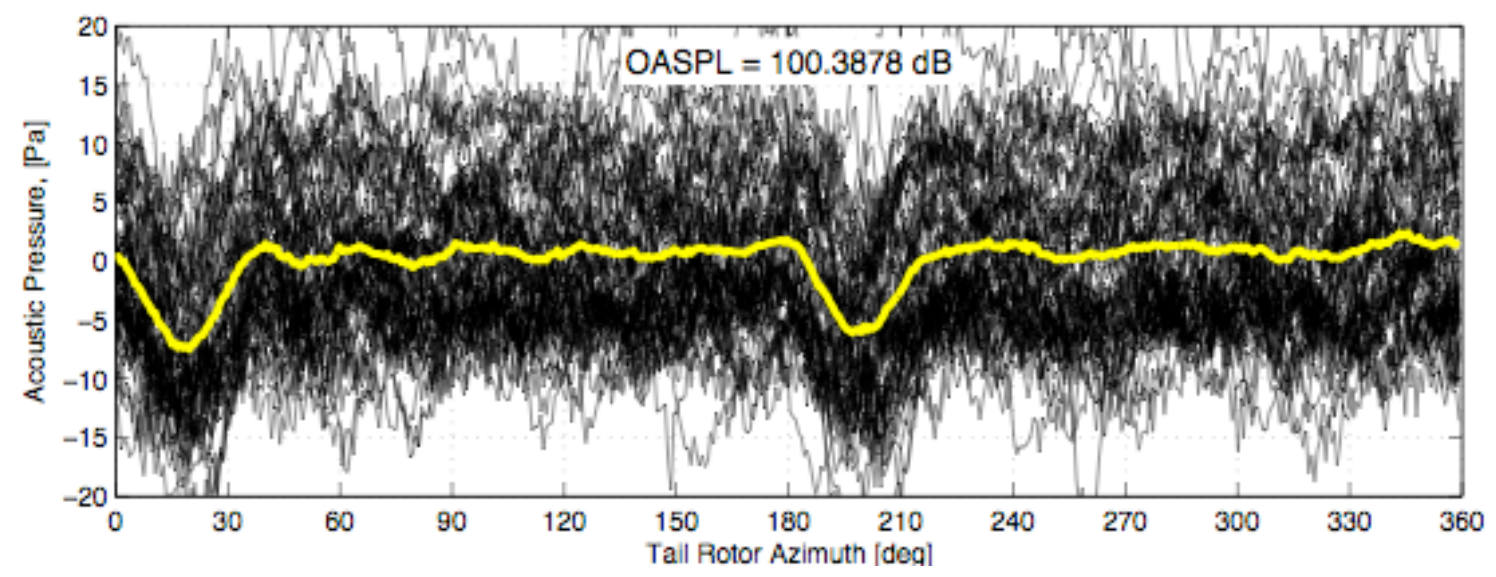

(f): A1 (- 12 degrees)

Figure 4.4: Variation of Microphone A1 with Descent Angle 
Figure 4.5 (a) through (f) show this reduction in loading noise at one of the outer microphones (A3). Also note that the amplitude range of the unfiltered data (in black) is significantly larger in these plots, despite the use of an $80 \mathrm{~Hz}$ filter. This is indicative of a significant amount of main rotor BVI (which is at frequencies comparable to the tail rotor harmonics). Despite the existence of this BVI however, the averaging process is still able to obtain the tail rotor time history. This is because the BVI noise being produced is still aperiodic with respect to the tail rotor revolution rate, and thus, is naturally averaged out when using the time averaging process.

The change in OASPL for all microphones as a function of descent angle is shown in Figure 4.6. All near in-plane microphones measure a decrease in OASPL of approximately $2.5 \mathrm{~dB}$ as descent angle increases. This change is quite slow and appears to be approaching a state of equilibrium as $-12^{\circ}$ is reached. The reason for the decrease in these microphones, despite their very small contributions from loading noise, is associated with the relative change in azimuth angle that the observer location is placed at as the descent angle changes. This is discussed more thoroughly in Chapter 6, where the comparison between theory and experiment is made.

There is a much more abrupt and significant change for the out-of-plane microphones. A dramatic change in OASPL $(\sim 3 \mathrm{~dB})$ occurs between $-3^{\circ}$ and $-6^{\circ}$, at which point the OASPL appears to level off and change in a manner similar to the near in-plane microphone noise levels. This difference can likely be attributed to the change in tail rotor loading that occurs at this descent angle and the dominance of loading noise that 


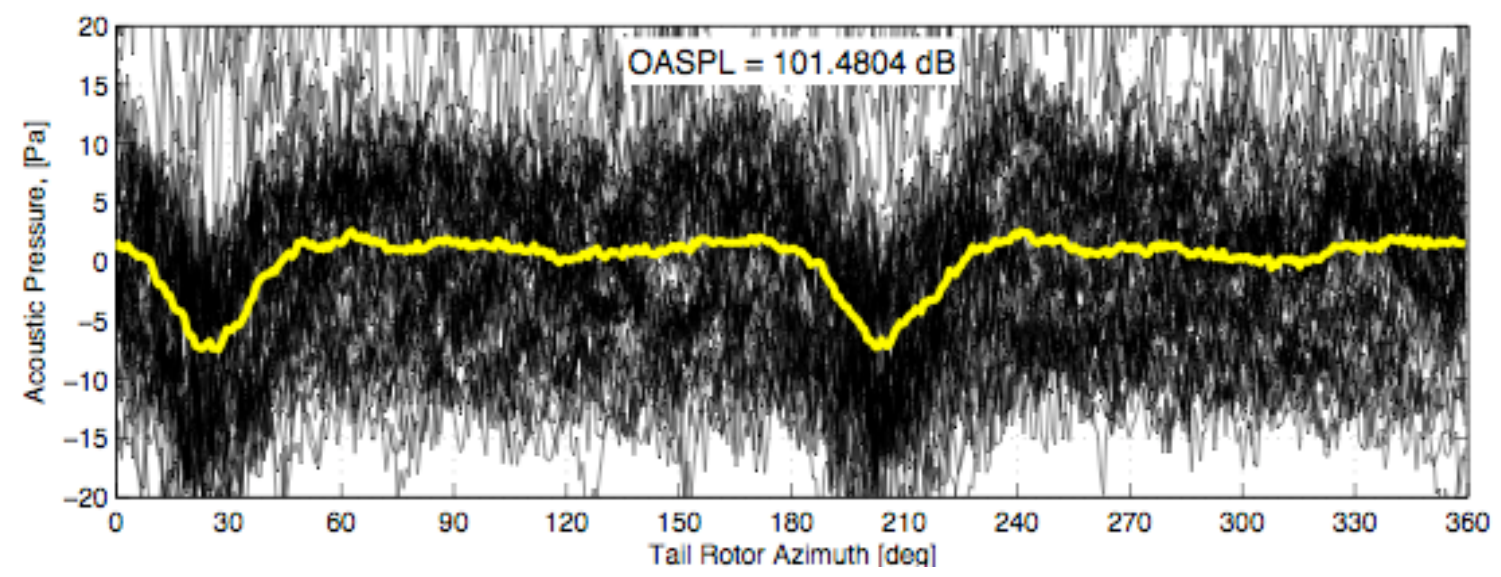

(a): $A 3$ (0 degrees)

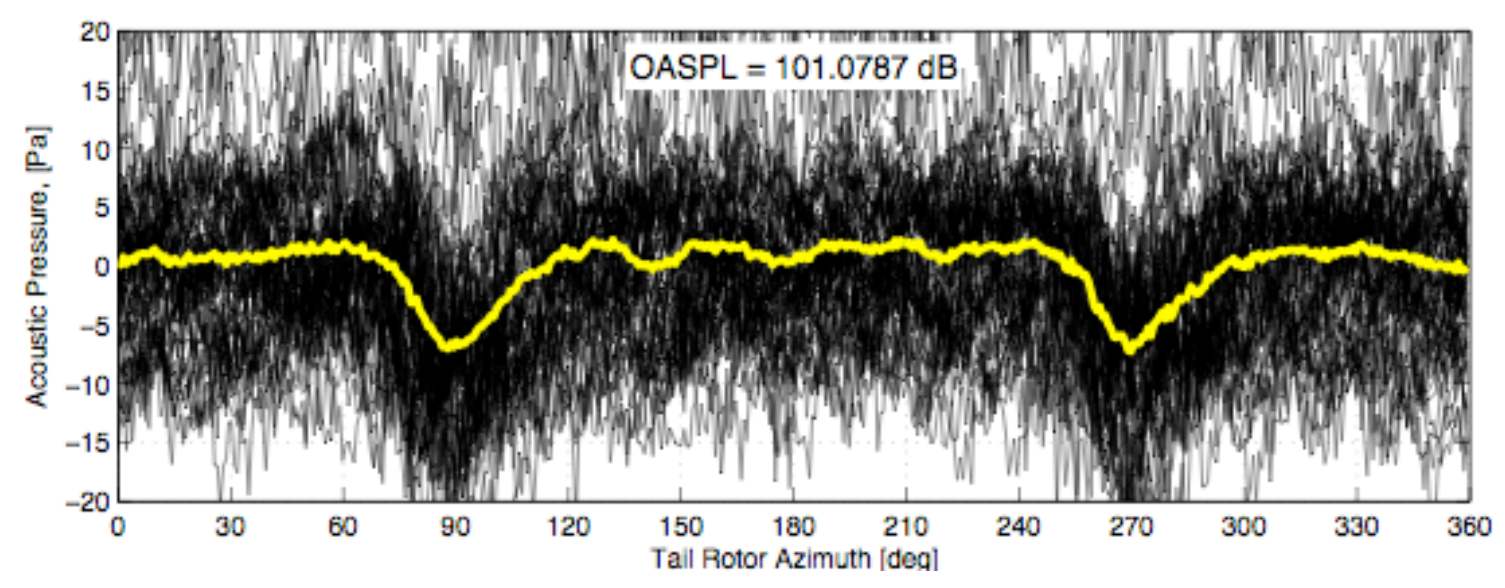

(b): A3 (-3 degrees)

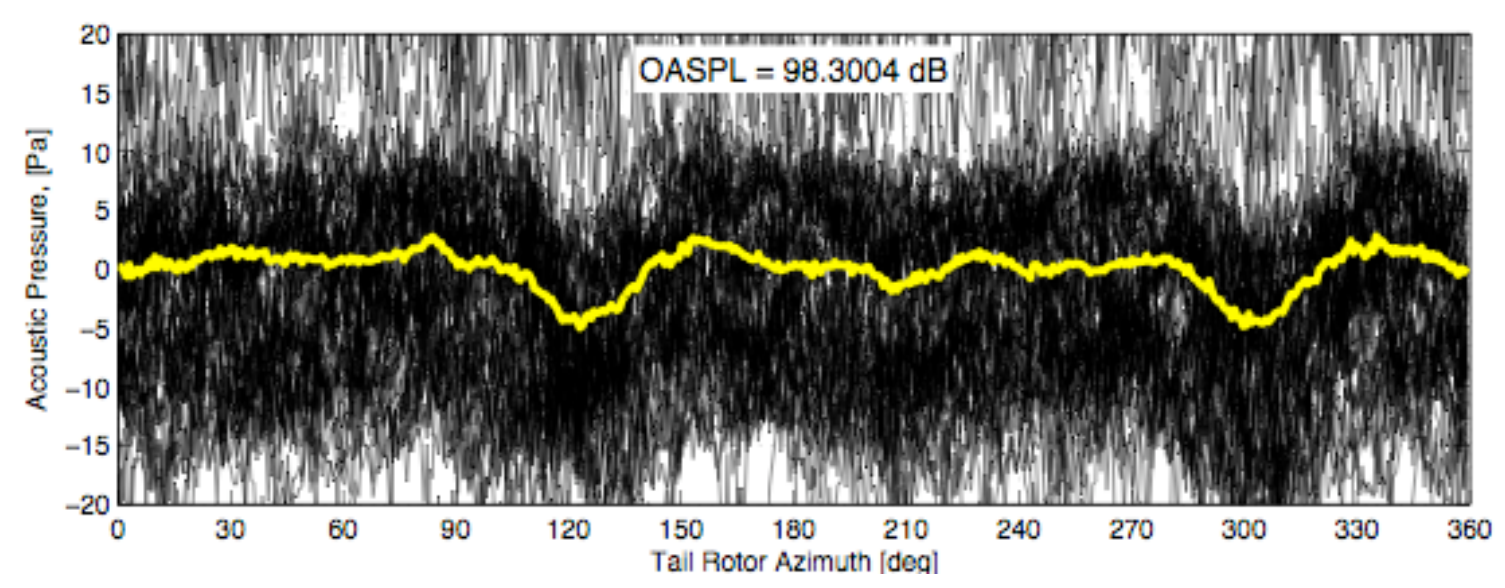

(c): A3 (-6 degrees) 


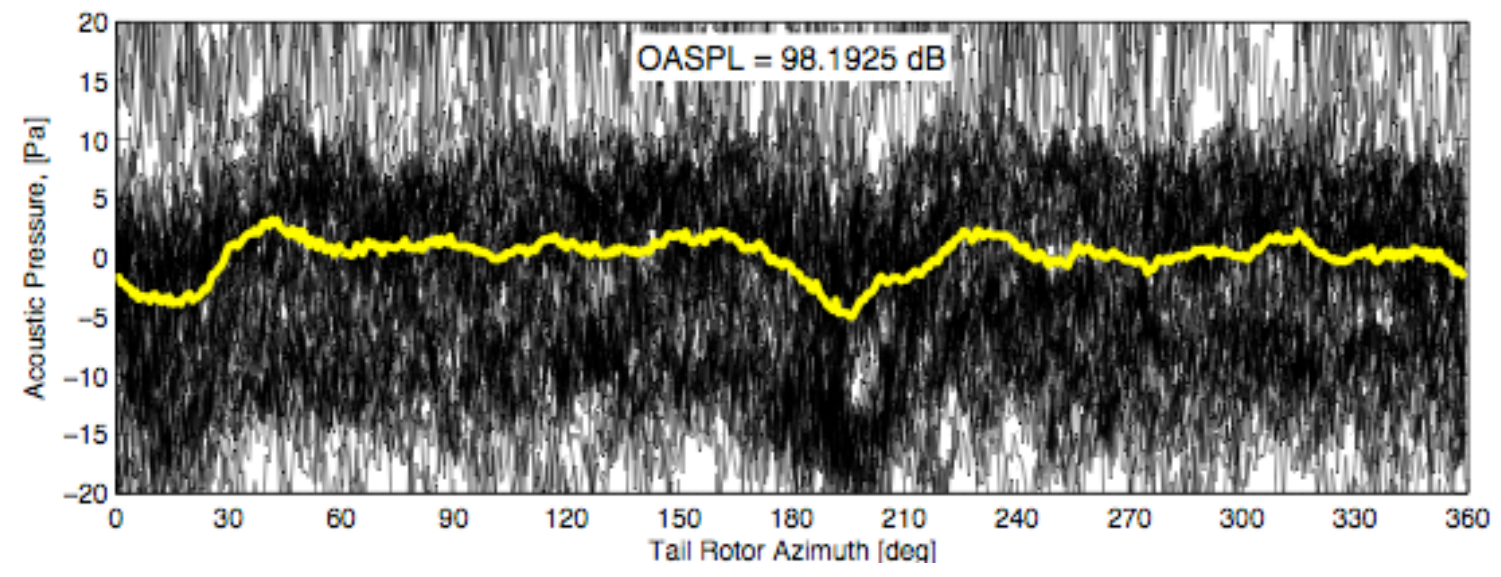

(d): $A 3(-7.5$ degrees $)$

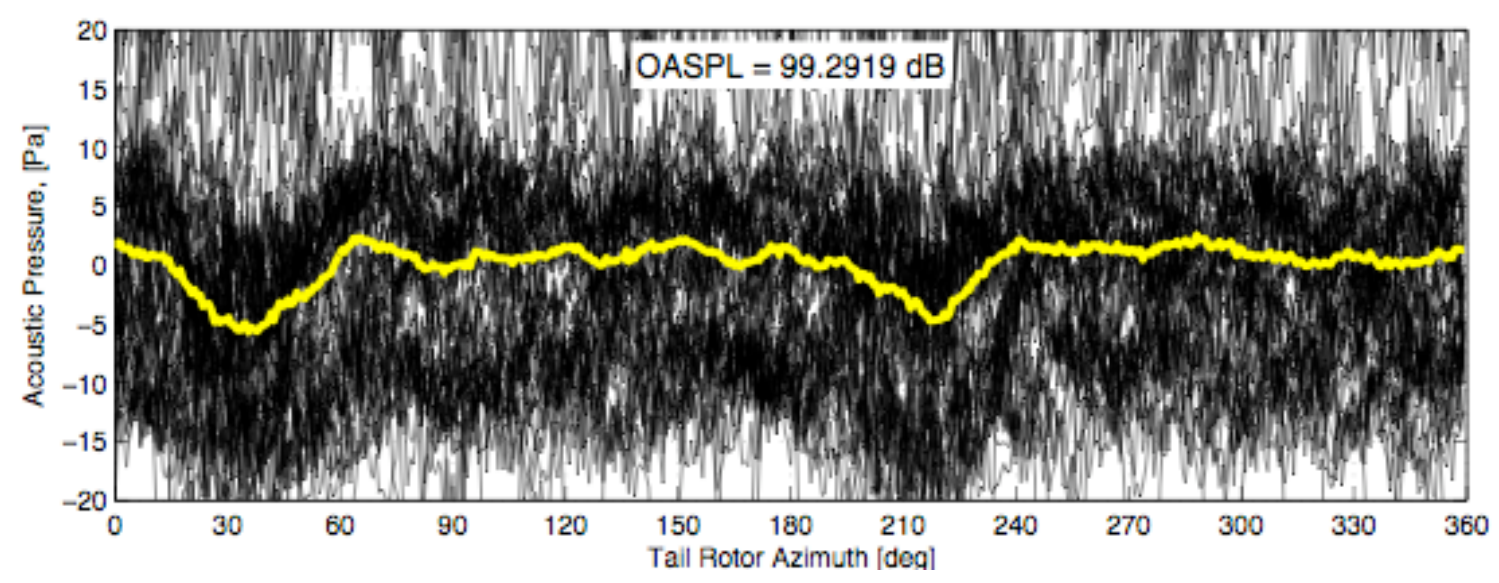

(e): $A 3$ (-9 degrees)

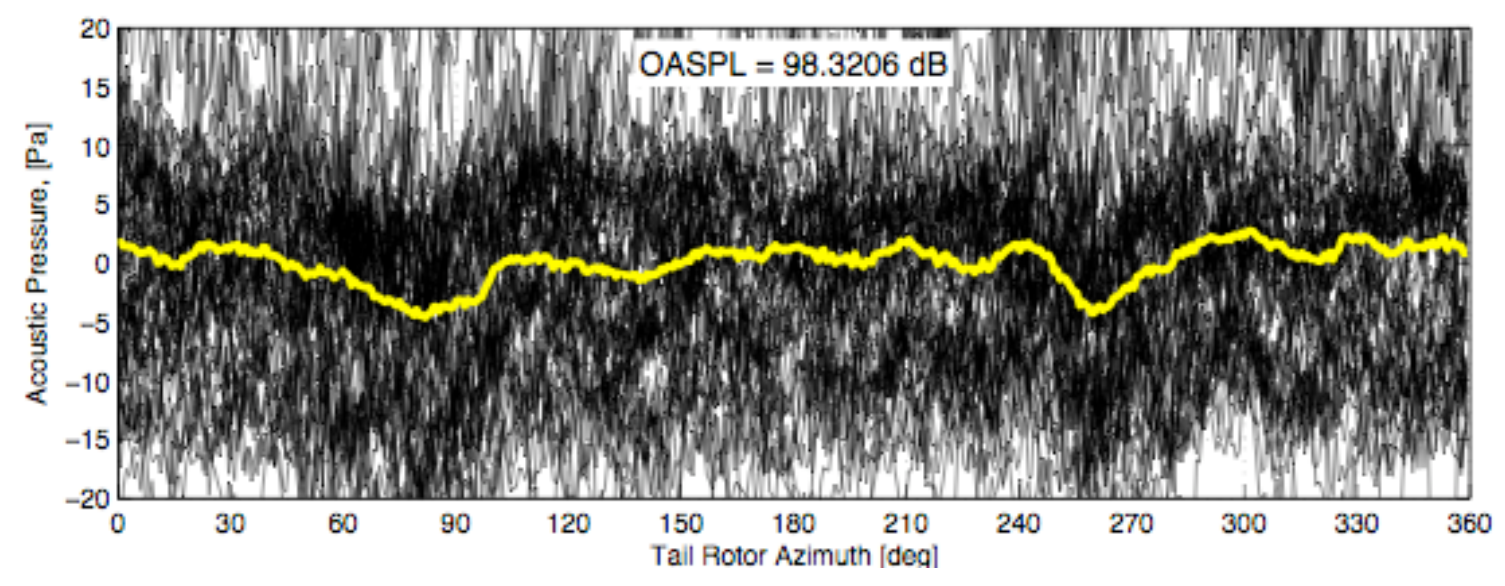

(f): $A 3$ (-12 degrees)

Figure 4.5: Acoustic time average at microphone $\mathrm{A} 3$ as a function of descent angle 
characterizes the microphone positions. It could also be due to a different type of loading on the fin or the tail rotor that occurs at this descent angle. Beyond $-6^{\circ}$ it appears as though the tail rotor is sufficiently offloaded so as to produce time histories dominated by thickness noise.

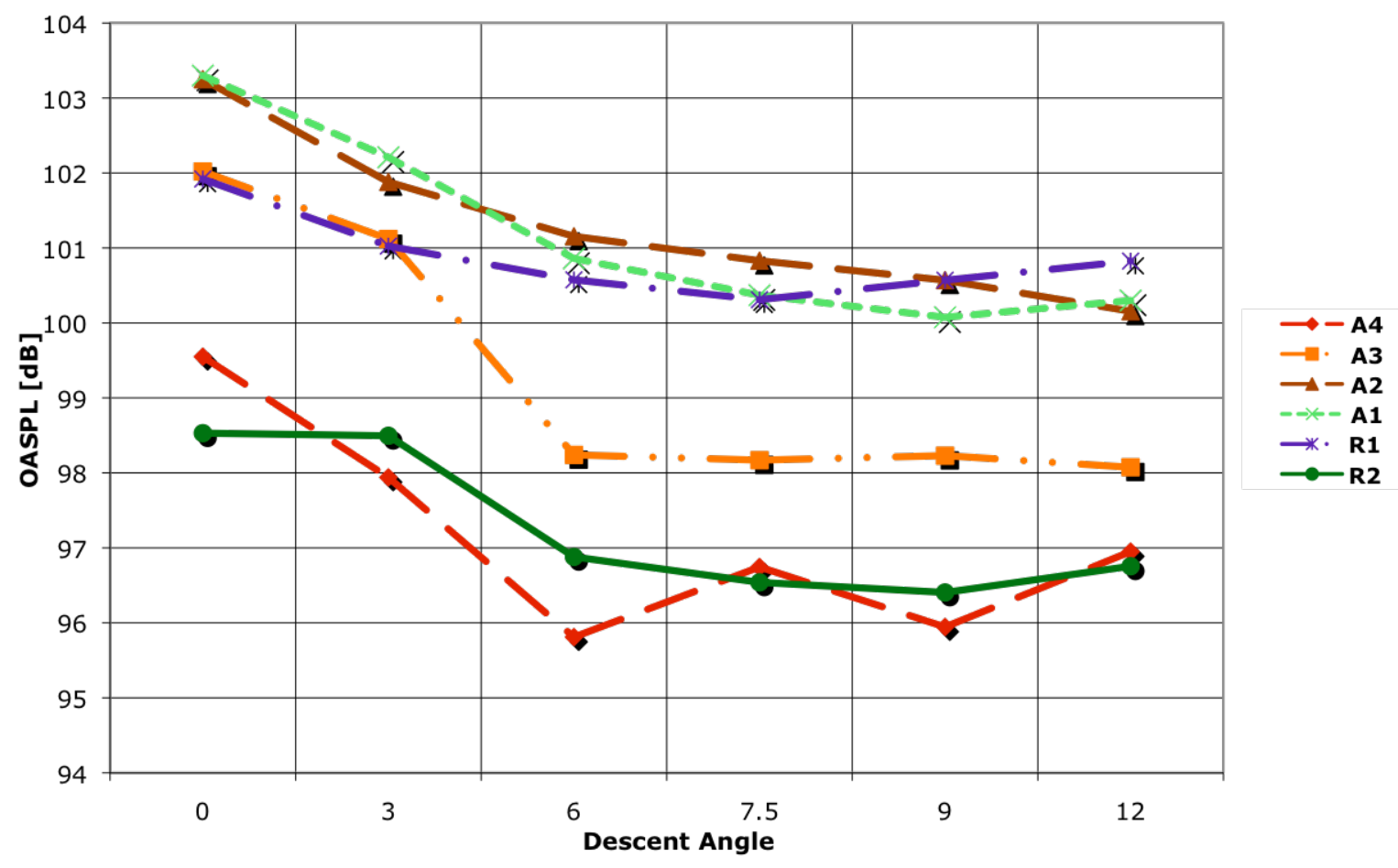

Figure 4.6: Variation in OASPL as a function of descent angle for all microphones 


\section{Chapter 5 Theoretical Tail Rotor Model}

\subsection{Summary}

Having good quality, time averaged, tail rotor acoustic data over a wide range of flight conditions presented a unique opportunity to validate harmonic noise theory. A relatively simple analysis was used for the comparison with the experimentally obtained time averages. This analysis was based upon a formulation of the Ffowcs Williams and Hawkings (FWH) equation. In addition to discussing the merits of using the FWH equation for the prediction of rotor noise, this chapter will discuss the method of implementation for both thickness noise as well as loading noise. A full explanation of the loading model that was developed to represent the tail rotor of the Bell 206B will also be discussed.

\subsection{Rotor Harmonic Noise Prediction}

The acoustic pressure generated by a rotor in the far-field is the perturbation aerodynamic pressure, which is governed by the fundamental laws of mass, momentum and energy. Though a full solution to the Navier-Stokes equations over the entire flow field would provide a solution to this pressure, the computations involved with this method exceed the capabilities of most modern computers when used for the rotor problem. ${ }^{29}$

In an effort to decouple the flow problem from the acoustic one, Lighthill rewrote the Navier-Stokes equations in order to force the appearance of a wave operator. This approach greatly reduced the demands for computational power. However, several 
limiting hypotheses associated with this method (it was derived for use in jet noise) make it less useful for application to the rotor problem. ${ }^{30}$

A similar approach was taken by Ffowcs Williams and Hawkings ${ }^{31}$ to reform the continuity equation and the Navier-Stokes equations into the form of a non-homogenous wave equation with three source terms. ${ }^{32}$ They used the idea that the conservation laws in differential form are also valid when all ordinary derivatives are viewed as generalized derivatives, which, when applied to general surfaces in motion, provide a governing equation which is more useful in rotor noise prediction.

$\square^{2} p^{\prime}=\frac{\partial}{\partial t}\left[\rho_{0} \nu_{n} \delta(f)\right]-\frac{\partial}{\partial x_{i}}\left[p n_{i} \delta(f)\right]+\frac{\partial^{2}}{\partial x_{i} \partial x_{j}}\left[H(f) T_{i j}\right]$

where $\square^{2}$ is the wave operator. In this derivation, the aerodynamic surface was assumed to be impenetrable. The monopole term in Equation 5.1 (first term on right) represents the thickness noise generated by the rotor, that is, the noise generated by the displacement of the fluid as the blade passes. The dipole term (second term) represents the loading noise generated through the steady and unsteady aerodynamic forces exerted on the fluid by the rotor. The third and final term, the quadropole term, models the nonlinearities attributed to the local flow field surrounding the tail rotor blades. ${ }^{34}$ This term is required for the accurate prediction of HSI noise, which, as discussed in Chapter 1, is normally of lesser importance to the tail rotor problem.

Whereas the FWH equation (5.1) is applicable only for a stationary observer, a different formulation is required to model the case that most closely resembles the 2006 flight test. This is a case in which the observer position is moving in conjunction with the source 
(similar to a wind tunnel environment). Through a slight modification of the retarded time equation, Farassat's Formulation 1 of the FWH equation, ${ }^{33}$ which has been successfully used for helicopter rotor and propeller noise prediction for many years, can be used to solve a problem with both a moving observer and source. Through the use of the free-space Green's theorem and the fact that the FWH equation is valid over unbounded space, the integral solution to Equation 5.1 can be found.

$$
4 \pi p^{\prime}(\vec{x}, t)=\underbrace{\frac{\partial}{\partial t} \int_{S}\left[\frac{\rho_{0} \nu_{n}}{r\left|1-M_{r}\right|}\right]_{\tau} d S}_{\text {Thickness }}+\underbrace{\frac{\partial}{\partial t} \int_{S}\left[\frac{P_{n} \cdot \hat{r}}{a_{0} r\left|1-M_{r}\right|}\right]_{\tau} d S}_{\text {Loading }}
$$

where $p^{\prime}(\vec{x}, t)$ is the perturbation pressure, $\mathrm{r}$ is distance between source and observer (magnitude of the radiation vector), $M_{r}$ is the relative Mach number of the source (as seen by the observer), $\rho_{0}$ is the density of the medium, $\mathrm{v}_{\mathrm{n}}$ is the velocity of the fluid normal to the surface of the blade, $P_{n} \cdot \hat{r}$ is the projection of the section lift pressure onto the radiation vector $r$, and $\mathrm{a}_{0}$ is the speed of sound in the medium. Neglecting the quadrupole term makes an additional simplification.

\subsubsection{Thickness Noise}

The first term of Equation 5.2 represents the thickness noise of the rotor and is due to the displacement of the medium by the blade. Thickness noise is a monopole source with a characteristic large negative pulse shape that is loudest in the place of the rotor. For this research this corresponds with microphones A1 and R1, located close to the tip-pathplane of the tail rotor. Figure 5.1 shows a representative thickness pulse for a two bladed tail rotor and confirms that the thickness noise is a maximum in the plane of the rotor. 


\section{Thickness}

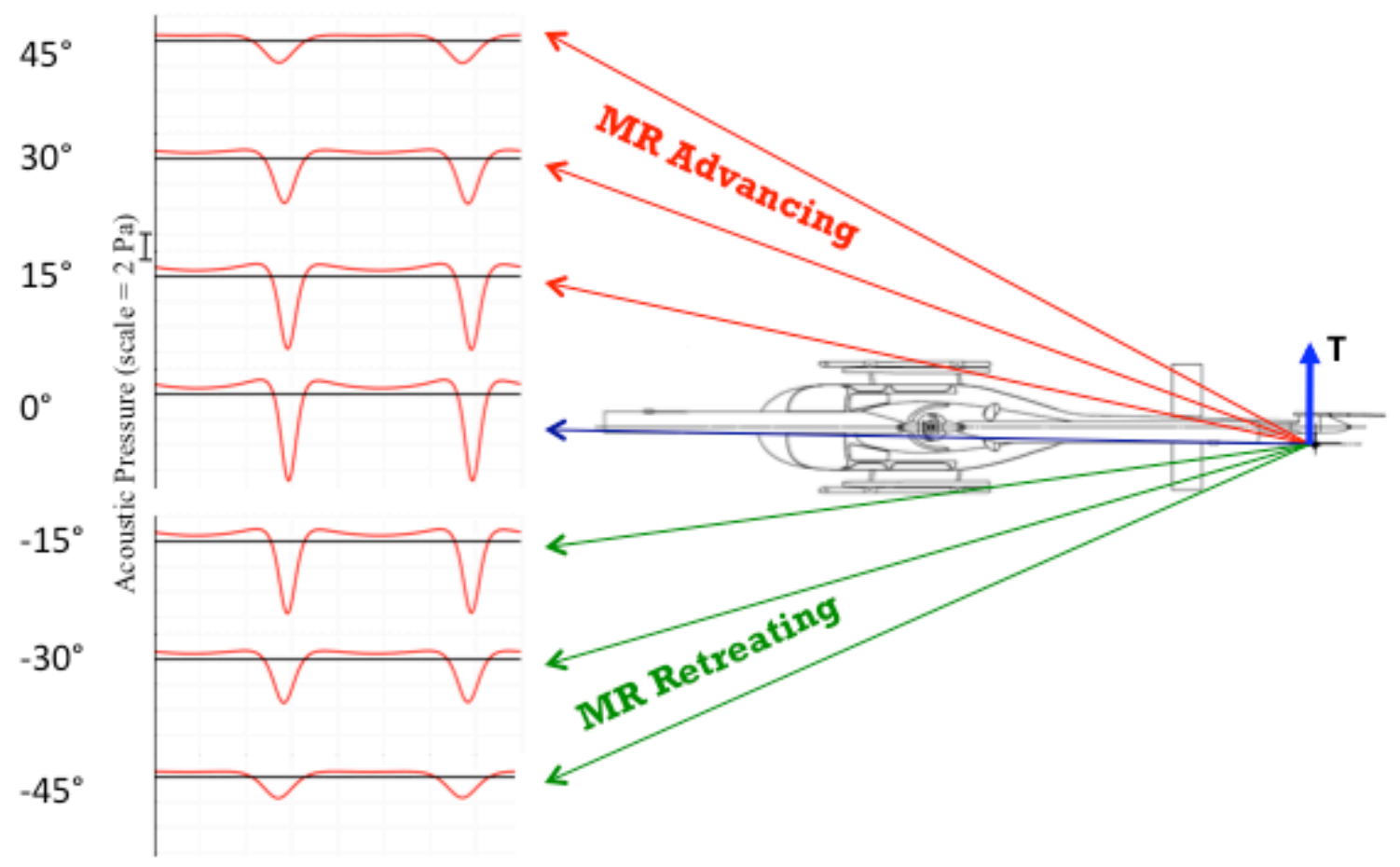

Figure 5.1: Thickness noise directivity and pulse shape for Bell 206B tail rotor

In order to solve for the thickness noise component of the acoustic pressure several assumptions were made. An air density of $1.2 \mathrm{~kg} / \mathrm{m}^{3}$ and speed of sound of $340.2 \mathrm{~m} / \mathrm{s}$ were both assumed. Each radial blade element was discretized into ten sources and ten sinks (on both the top and bottom surfaces, for a total of 40), with 16 span-wise elements per blade (from $0.2 \mathrm{R}$ to $1.0 \mathrm{R}$ with $0.05 \mathrm{R}$ width spans). The blade discretization is illustrated in Figure 5.2 for one blade. The black dots represent the nodes at which the acoustic pressure was computed. Finally, a rotation rate of $41.5 \mathrm{~Hz}$ was used for the thickness noise prediction 


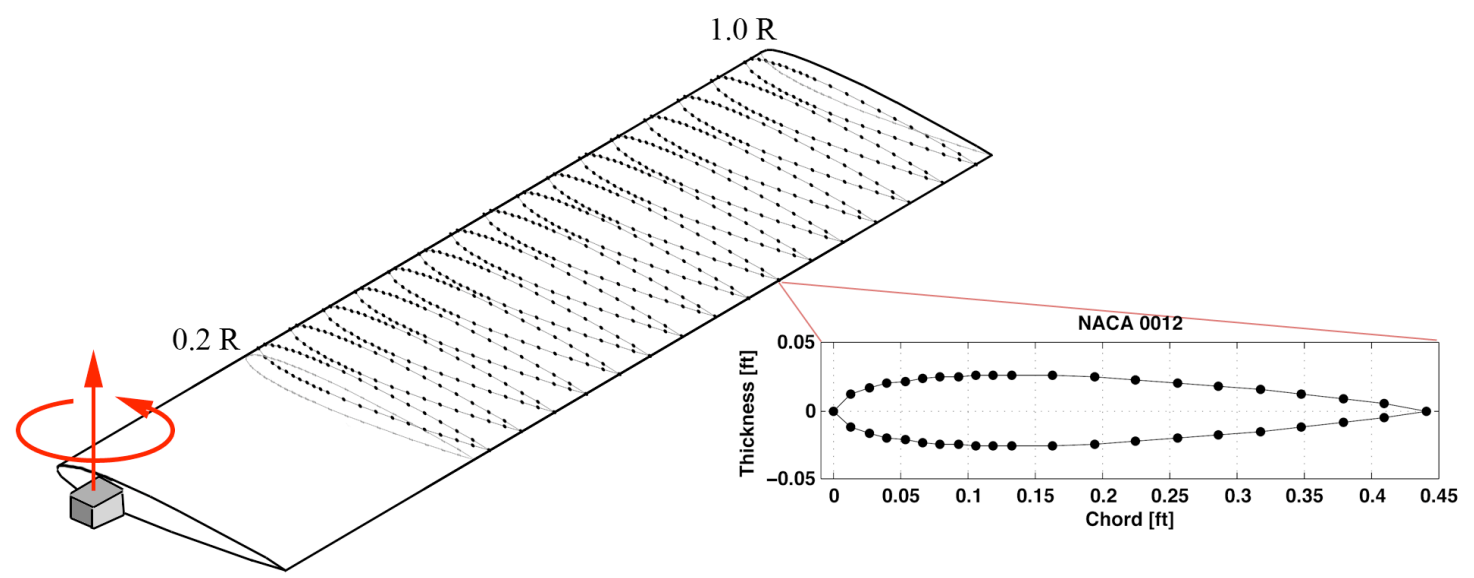

Figure 5.2: Blade discretization for FWH implementation of thickness noise

\subsubsection{Loading Noise}

The second term of Equation (5.2) represents the loading noise generated by the rotor. This noise is due to the aerodynamic pressure forces acting on the medium (air) and requires a separate calculation of the tail rotor air-loads at each flight condition. These air-loads were estimated by developing a simple tail rotor-loading model that is discussed in Section 5.3. This model provides a first-order estimate of the tail rotor loads using blade element momentum theory. Figure 5.3 shows the directivity pattern and representative pulse shape for the loading noise produced by the tail rotor of the Bell 206B.

The prediction for loading noise was computed independently of the thickness noise calculation and it wasn't until after both had been computed that they were summed. Because of this, different assumptions and discretization could be made for the tail rotor loading model independently of those that were made for thickness model. 


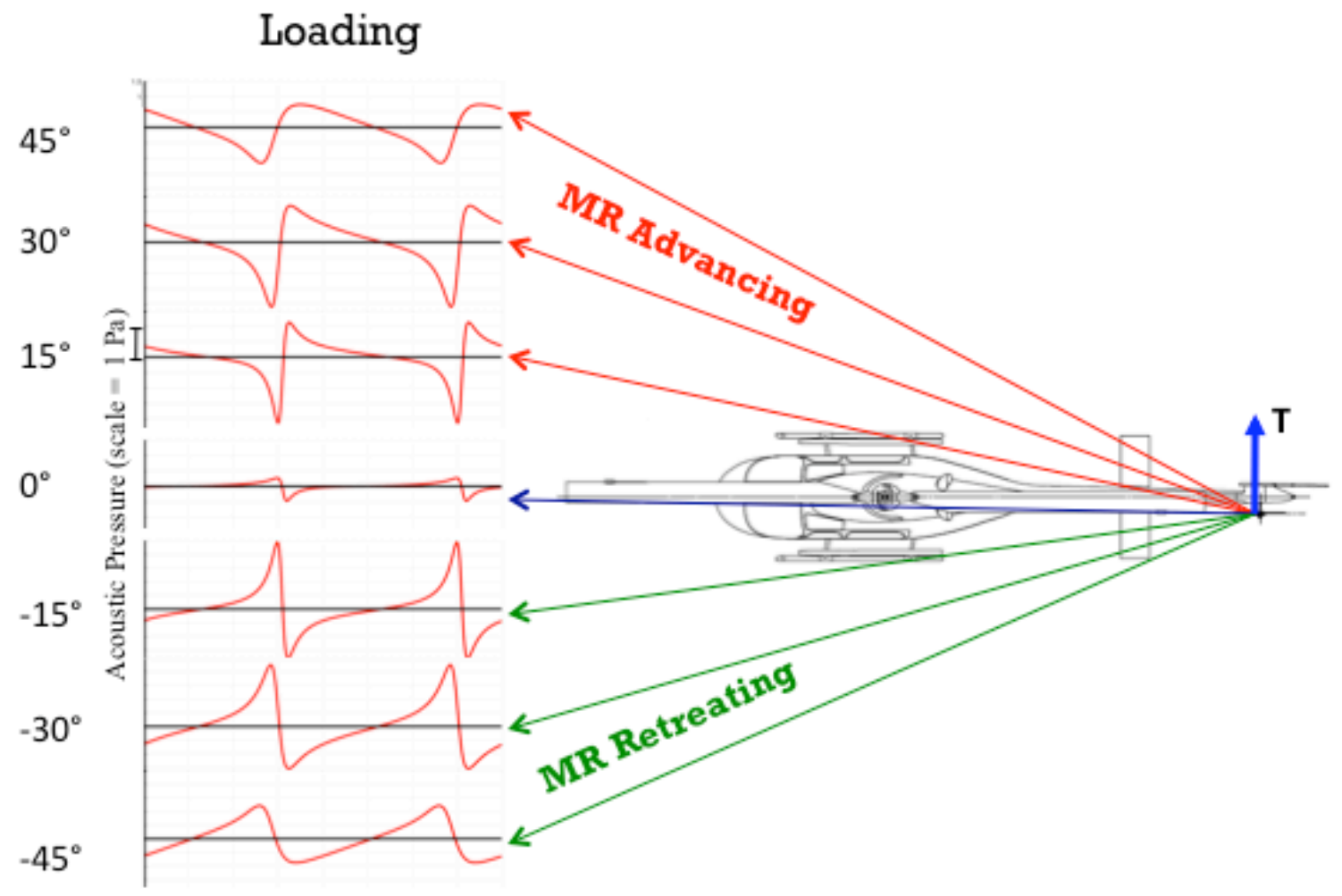

Figure 5.3: Loading noise directivity and pulse shape for Bell 206B tail rotor

\subsection{Tail Rotor Loading Model}

Several assumptions were made in the estimation of the blade loading of the tail rotor. The tail rotor of the $206 \mathrm{~B}$ was modeled as a flapping, teetering, untwisted, two-bladed rotor with collective input and a $\delta_{3}$ angle of $45^{\circ}$ for pitch-flap coupling. The flapping and collective angles were calculated using a simple blade element momentum theory analysis (BEMT) based in the tip path plane of the tail rotor and made with a uniform inflow assumption. ${ }^{35,36}$ A compact chord lifting line theory assumption was made for the calculation of the blade loads and each blade was discretized into 20 radial sections. The tail rotor shaft angle was assumed to be $0^{\circ}$, that is, the helicopter was assumed to be flying with no sideslip angle. Finally, the entirety of the main rotor torque was assumed 
to be counteracted by the tail rotor, with no off-loading due to the lift generated by the vertical fin. This assumption is investigated further in Chapter 6.

\subsubsection{Calculation of Tail Rotor Thrust}

The first step in the calculation of the tail rotor air-loads was the estimation of the required tail rotor thrust. Keeping in mind that the entirety of the main rotor torque was balanced by the tail rotor, momentum theory tells us,

$$
C_{P}=C_{P_{i}}+C_{P_{o}}+C_{P_{p}}+C_{P_{c}}
$$

Where the first term in (5.3) represents the induced power coefficient,

$$
C_{P_{i}}=\frac{\kappa C_{W}^{2}}{2 \sqrt{\lambda^{2}+\mu^{2}}}
$$

with the induced power factor $\kappa \approx 1.15$ and the weight coefficient $C_{W}=\frac{W}{\rho A(\Omega R)^{2}}$ with an approximate weight of the Bell 206B being $3200 \mathrm{lb}$.

The uniform inflow was calculated in the tip path plane using,

$$
\lambda=\mu \tan \alpha_{T P P}+\frac{C_{W}}{2 \sqrt{\mu^{2}+\lambda^{2}}}
$$

where $\alpha_{T P P} \approx \frac{D}{W}=\frac{\frac{1}{2} \rho\left(V_{\infty}\right)^{2} f}{W}$ with the flat plate area of the helicopter, including boom microphone array, being $f \approx 14 f t^{2}$.

The second term in (5.3) represents the profile power coefficient and is equal to, 


$$
C_{P_{O}}=\frac{\sigma C_{d_{o}}}{8}\left(1+4.66 \mu^{2}\right)
$$

This was calculated using a main rotor section profile drag coefficient of $C_{d_{o}}=0.01$.

The third term in (5.3) is the parasitic power required, that is,

$$
C_{P_{p}}=\frac{1}{2}\left(\frac{f}{A}\right) \mu^{3}
$$

The fourth and final term of (5.3) is the amount of power required to climb (or in this case descend), and, using the small angle assumption, is equal to,

$$
C_{P_{C}}=\frac{(W)\left(v_{C}\right)}{\rho A(\Omega R)^{3}}
$$

where $\mathrm{v}_{\mathrm{c}}$ is the climb velocity of the helicopter.

Upon calculating the main rotor power for a given speed and descent angle, the tail rotor thrust could be estimated,

$$
T_{T R}=\frac{\rho A(\Omega R)^{3}\left(C_{P_{i}}+C_{P_{o}}+C_{P_{P}}+C_{P_{C}}\right)}{\Omega X_{T R}}
$$

where the distance between the main rotor shaft and the tail rotor was $X_{T R} \approx 19.48 \mathrm{ft}$. The main rotor tip speed was also approximated to be $\Omega \mathrm{R}_{\mathrm{MR}}=688 \mathrm{ft} / \mathrm{s}$.

The tail rotor thrust required for the Bell 206B flying at 63 knots, steady level flight was found to be $494 \mathrm{~N}$. 


\subsubsection{Tail Rotor Trim}

The trim process for a tail rotor of this type is simpler than the conventional main rotor trim process. The model used for this work was a structurally rigid, two-bladed rotor with a teetering hub and $\delta_{3}$ hinge for pitch-flap coupling to minimize flapping. Blade element momentum theory was used to calculate the collective input as well as the flapping motion of the rotor. This process began with the balancing of the thrust, pitching moment and rolling moment equations.

$$
\begin{aligned}
& C_{T_{T R}}=\frac{\sigma C_{l \alpha}}{4 \pi} \int_{0}^{2 \pi} \int_{0}^{1}\left(\bar{U}_{T}^{2} \theta-\bar{U}_{P} \bar{U}_{T}\right) d r d \psi \\
& C_{M_{X}}=0=\frac{\sigma C_{l \alpha}}{4 \pi} \int_{0}^{2 \pi} \int_{0}^{1} r \sin \psi\left(\bar{U}_{T}^{2} \theta-\bar{U}_{P} \bar{U}_{T}\right) d r d \psi \\
& C_{M_{Y}}=0=-\frac{\sigma C_{l \alpha}}{4 \pi} \int_{0}^{2 \pi} \int_{0}^{1} r \cos \psi\left(\bar{U}_{T}^{2} \theta-\bar{U}_{P} \bar{U}_{T}\right) d r d \psi \\
& \bar{U}_{T}(r, \psi)=r+\mu \sin \psi \\
& \bar{U}_{P}(r, \psi)=\lambda_{T R}+\frac{r \dot{\beta}}{\Omega}+\mu \beta \cos \psi \\
& \theta(r, \psi)=\theta_{0}-\beta_{1 c} \tan \delta_{3} \cos \psi-\beta_{1 s} \tan \delta_{3} \sin \psi \\
& \beta(r, \psi)=\beta_{1 c} \cos \psi+\beta_{1 s} \sin \psi
\end{aligned}
$$

Though a more complex inflow model could have been used at this point, it was determined that the use of a linear inflow model provided no additional proximity to the actual inflow environment that a tail rotor experiences. Since this model served only as a first order comparison with experimental data, it was well beyond the scope of this research to develop a more complex inflow model. Therefore, assuming uniform inflow, the inflow at the tail rotor was assumed to be, 


$$
\lambda_{T R}=\underbrace{\mu_{T R} \tan \alpha_{T R}}_{=0}+\frac{C_{T_{T R}}}{2{\sqrt{{\mu_{T R}}^{2}+\lambda_{T R}^{2}}}^{2}}
$$

using small angle assumptions for the tail rotor shaft angle.

Then, solving the equilibrium equation yields the trim system of equations,

$$
\left[\begin{array}{ccc}
\pi \mu^{2}+\frac{2 \pi}{3} & 0 & -\pi \mu \tan \delta_{3} \\
\frac{2 \pi}{3} \mu & -\frac{\pi}{8} \mu^{2}+\frac{\pi}{4} & -\left(\frac{3 \pi}{8} \mu^{2}+\frac{\pi}{4}\right) \tan \delta_{3} \\
0 & \left(\frac{\pi}{8} \mu^{2}+\frac{\pi}{4}\right) \tan \delta_{3} & \frac{\pi}{8} \mu^{2}+\frac{\pi}{4}
\end{array}\right]\left[\begin{array}{c}
\theta_{0} \\
\beta_{1 c} \\
\beta_{1 s}
\end{array}\right]=\left[\begin{array}{c}
\frac{4 \pi C_{T_{T R}}}{\sigma C_{l \alpha}}+\lambda_{T R} \pi \\
\frac{\lambda_{T R} \pi \mu}{2} \\
0
\end{array}\right]
$$

Which can be solved to find the collective input and flapping motion of the tail rotor.

Representative values of the solution to this system of equations are shown below in Table 5.1 for the Bell 206B flying at 63 knots, steady level flight. The flapping motion of the rotor is shown in Figure 5.4. This model was validated in a comparison with results from Prouty and is included as an appendix to this work (Appendix B).

Table 5.1: Tail Rotor trim values (63 knots)

\begin{tabular}{|c|c|c|c|}
\hline Tail Rotor Thrust & Collective $\left(\theta_{0}\right)$ & Longitudinal $\left(\beta_{1 \mathrm{C}}\right)$ & Lateral $\left(\beta_{1 \mathrm{~S}}\right)$ \\
\hline $494.1868 \mathrm{~N}$ & $3.7351^{\circ}$ & $-0.6371^{\circ}$ & $0.6371^{\circ}$ \\
\hline
\end{tabular}




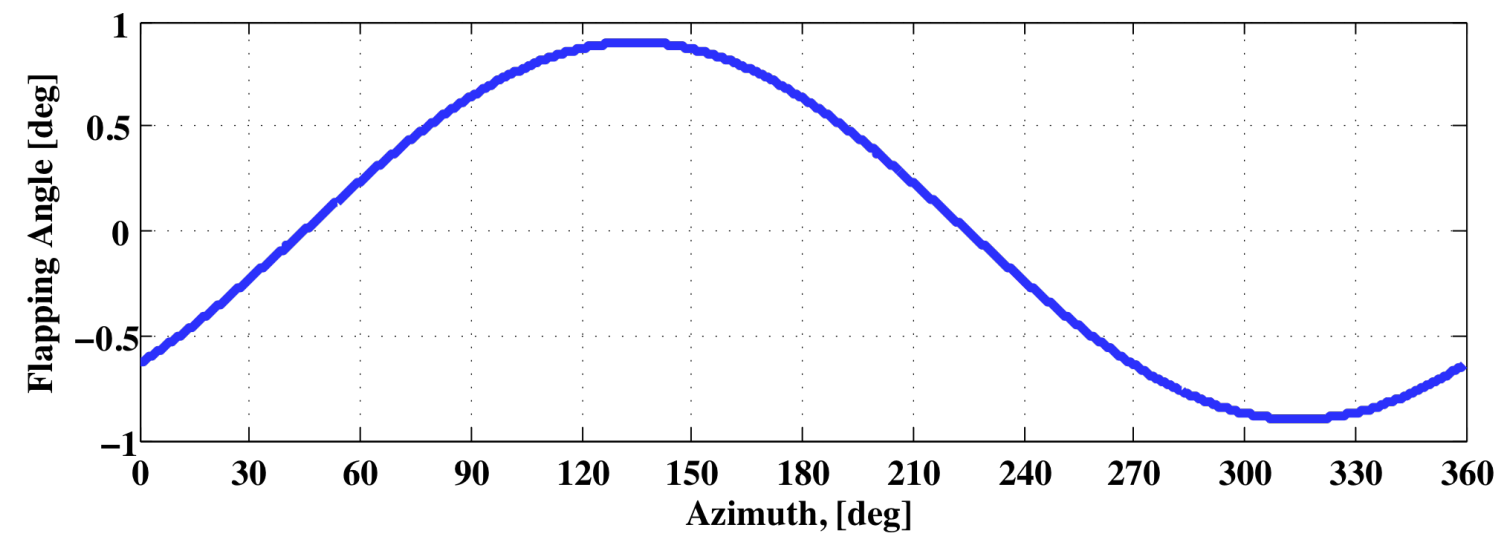

Figure 5.4: Flapping motion of tail rotor model (63 knots)

\subsubsection{Calculation of Tail Rotor Loading Distribution}

After solving the trim equation, the incremental lift produced by each radial element was,

$$
\Delta L=\frac{1}{2} \rho c C_{l \alpha}\left(\bar{U}_{T}^{2} \theta-\bar{U}_{P} \bar{U}_{T}\right) \Delta r
$$

This lift was approximated to be acting on the entire radial element at the quarter chord of the blade, at an angle $\phi=\tan ^{-1}\left(\frac{\bar{U}_{P}}{\bar{U}_{T}}\right)$ from the vertical access.

The calculated value of the out of plane force, with respect to the TPP of the tail rotor, is shown in Figure 5.5 for the 63 knot steady level flight case for several radial sections. 

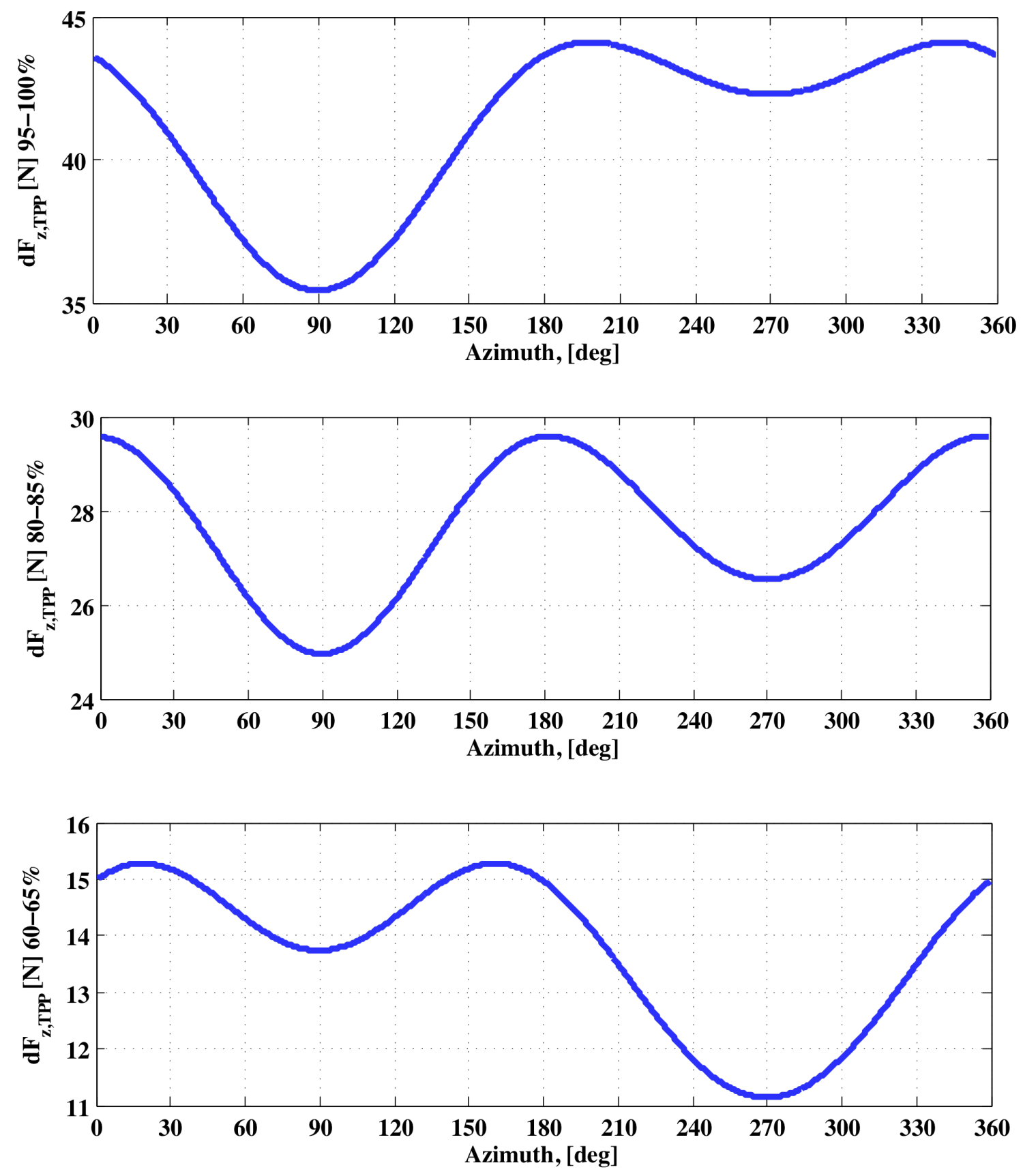

Figure 5.5: Out-of-Plane loading of the tail rotor model

The in-plane loading was also calculated using this method, and, as shown in Figure 5.6, is significantly smaller than the out-of-plane loading. For this purpose it was neglected. 

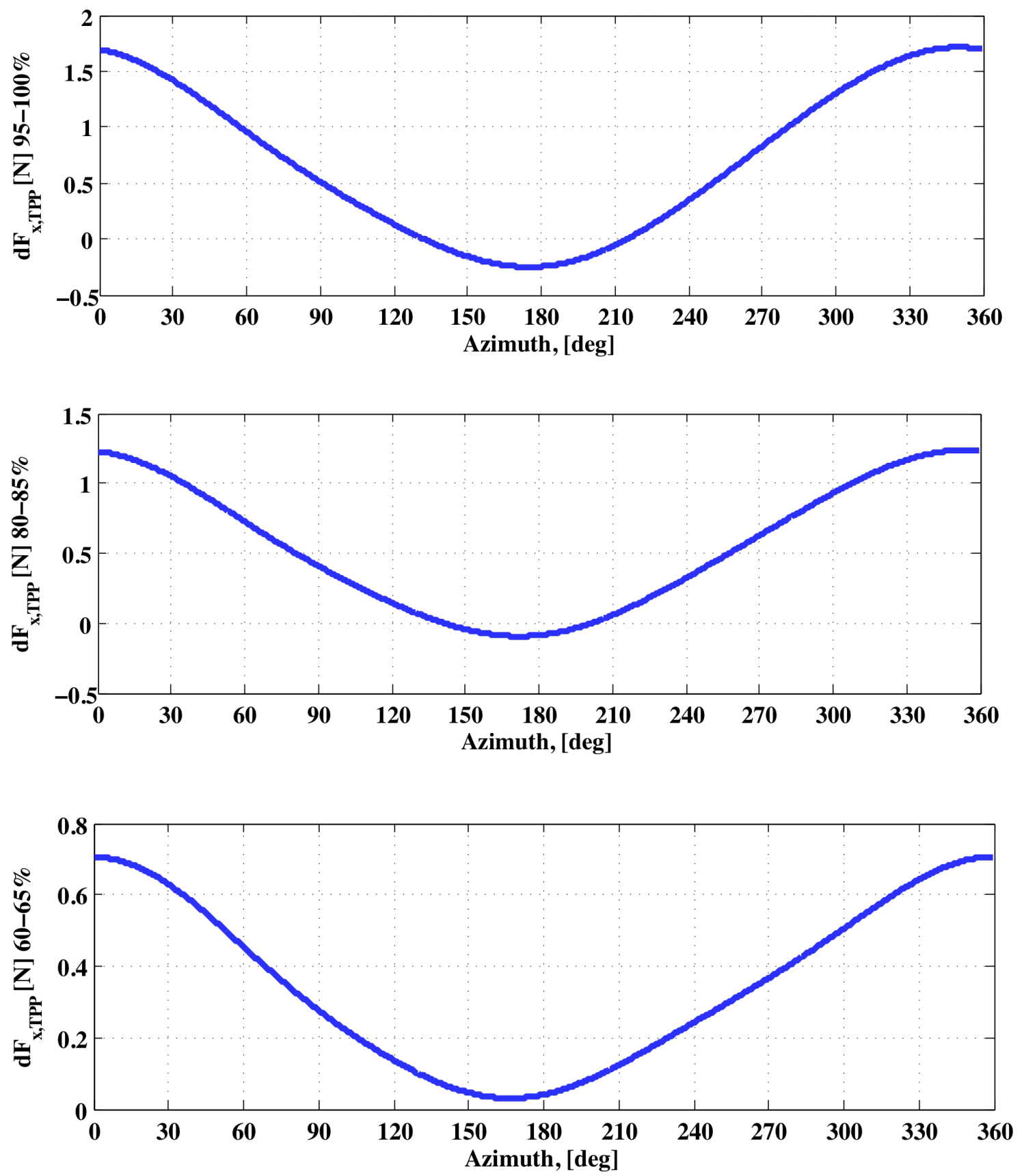

Figure 5.6 : In-plane loading of the tail rotor loading

The loading data generated with this method was then used in conjunction with the second term on the right hand side of Equation (5.3) to solve for the loading noise contribution of the tail rotor. 


\subsection{Total Theoretical Noise}

In addition to the pulse shape and amplitude of both the loading and thickness noise pulses, the relationship in phase between both sources is of the upmost importance. This importance stems from the affect that phasing has on the amplitude and shape of the resulting superposition. Because both sources were computed in the same time domain, the superposition is relatively straightforward. Figure 5.7 shows the directivity pattern of the combined thickness and loading noise. Note that while the loading noise reinforces the thickness pulse at positive elevation angles, it reduces the thickness pulse at negative elevation angles.

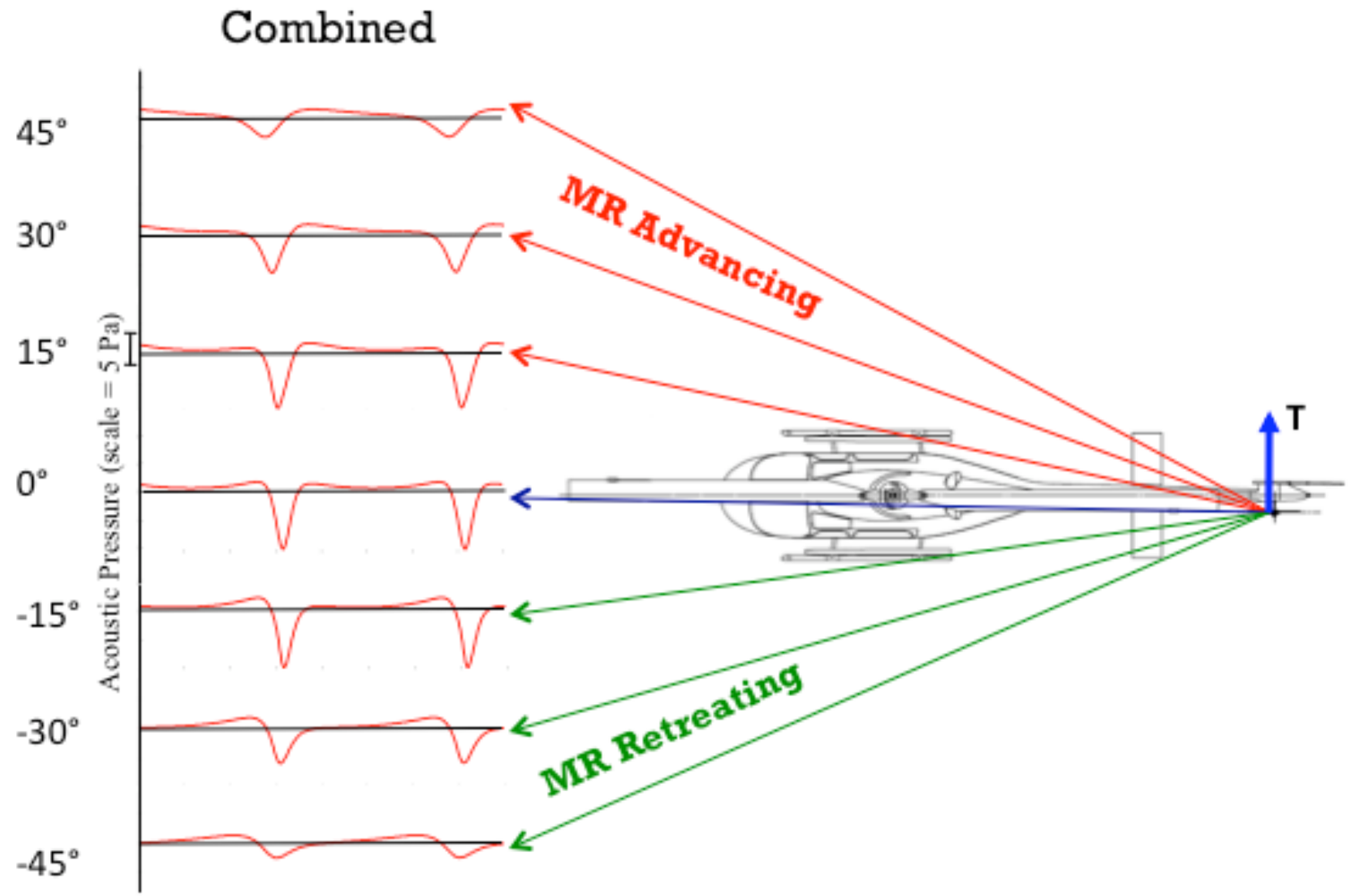

Figure 5.7: Theoretical Combined Loading and Thickness Noise 


\section{Chapter 6 Comparison with Theory}

\subsection{Summary}

Another way of assessing the influence of the contributions of thickness and loading on the radiated noise of a tail rotor is through a direct comparison of theory and experiment. This chapter presents this comparison using the experimental data obtained for the 63 knots level flight case and the theoretical model developed in Chapter 5. This comparison is made at all of the boom mounted microphone positions. An additional comparison is made for the -7 degree descending flight case. The theoretical prediction of the tail rotor noise has been calculated based on first principle acoustics (See Section 5.2).

\subsection{Steady Level Flight: Comparison with Theory}

Figure 6.1 shows the comparison between theory and experiment for all microphones for steady level flight at 63 knots. Overall, the comparison between theory and experiment is quite good. The microphone farthest out-of-plane on the starboard side of the helicopter, A4, shows reasonably good correlation between theory and experiment (Figure 6.1 (a)). Both the positive and negative peak amplitude is represented well, as is the low frequency pulse width. However, there is a slightly larger positive peak just before the negative peak in the time average measurements than is shown by theory. This same trend appears in Figure 6.1 (b) as the negative peak is well represented, with the leading positive peak being under predicted by theory. The comparison between theory and experiment improves for microphones A2 and A1 (Figure 6.1 (c) and (d)), with the differences becoming less pronounced on the near in-plane microphone positions. This 
observation suggests that loading noise is being over-predicted at the out-of-plane microphone positions. This loading noise over-prediction is likely due to an overprediction of tail rotor thrust though this is explored further in this chapter.

There is good agreement between theory and experiment for the near in-plane port side microphone, R1. Thickness noise is the dominant source of noise at this microphone position, and, as previously hypothesized, any loading noise contributions appear to have a subtractive affect on the total amplitude as compared with microphone A2. Similarly, at microphone R2, the loading noise appears to cancel out a considerable amount of the thickness noise, reducing the measured noise at R2 to very low levels.

Another point of interest in these figures is that the averages also appear to display evidence of some higher harmonic periodic loading noise. In Figure 6.1 (a) for instance, there are small oscillations after the major aperiodic pulse. This higher harmonic noise is likely due to the interaction between the tail rotor and the vertical fin either in the form of reflected noise or a changing load pattern. Though the tail rotor noise model developed in Chapter 5 does not incorporate these effects, they are of secondary importance with respect to the main thickness and loading pulses. The incorporation of these scattering and vertical fin effects could certainly be incorporated into later analysis and models for the tail rotor noise. 


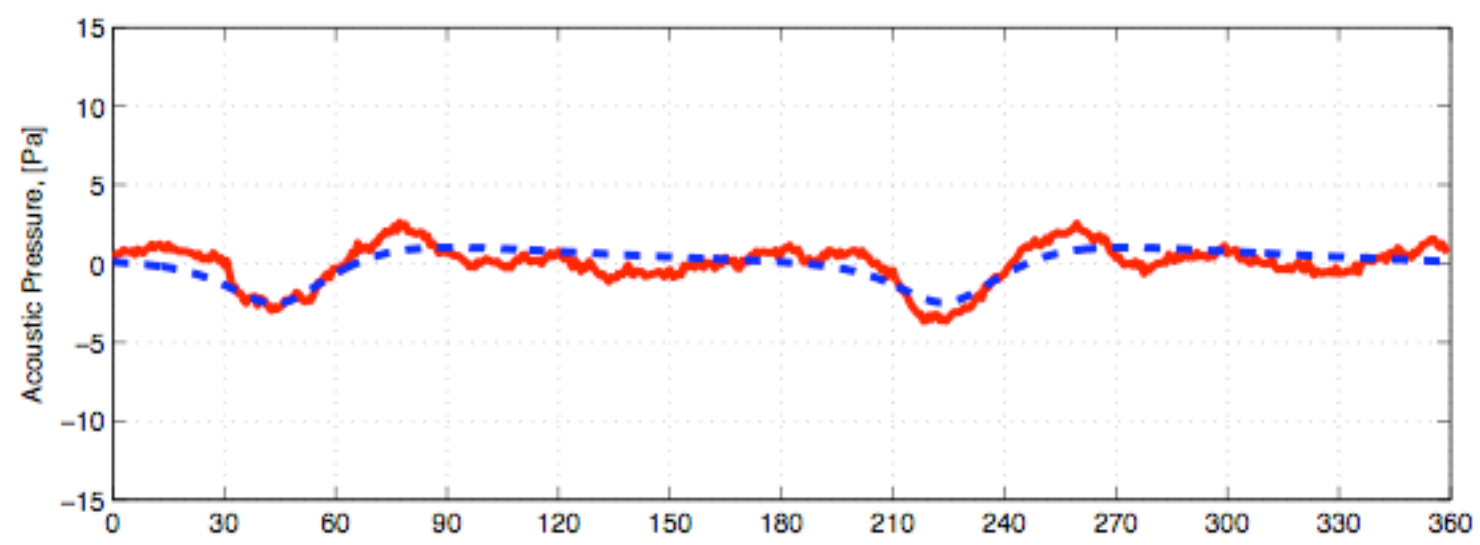

(a): $A 4$ (63 kts)

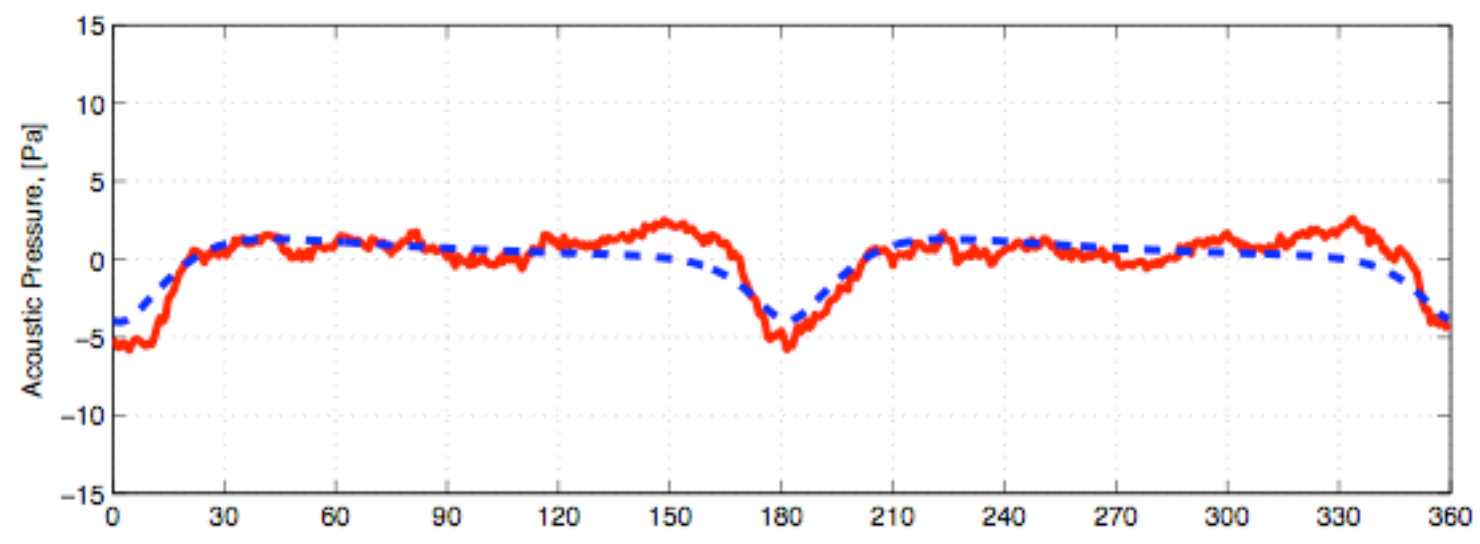

(b): $A 3(63 \mathrm{kts})$

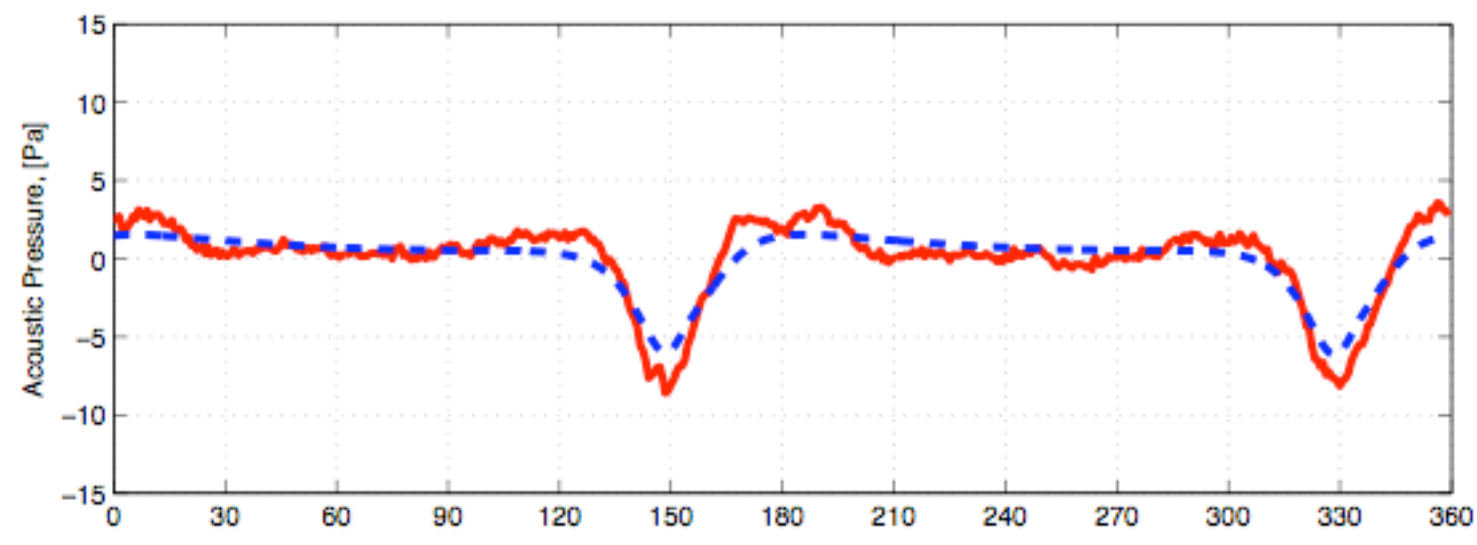

(c): $A 2(63 \mathrm{kts})$ 


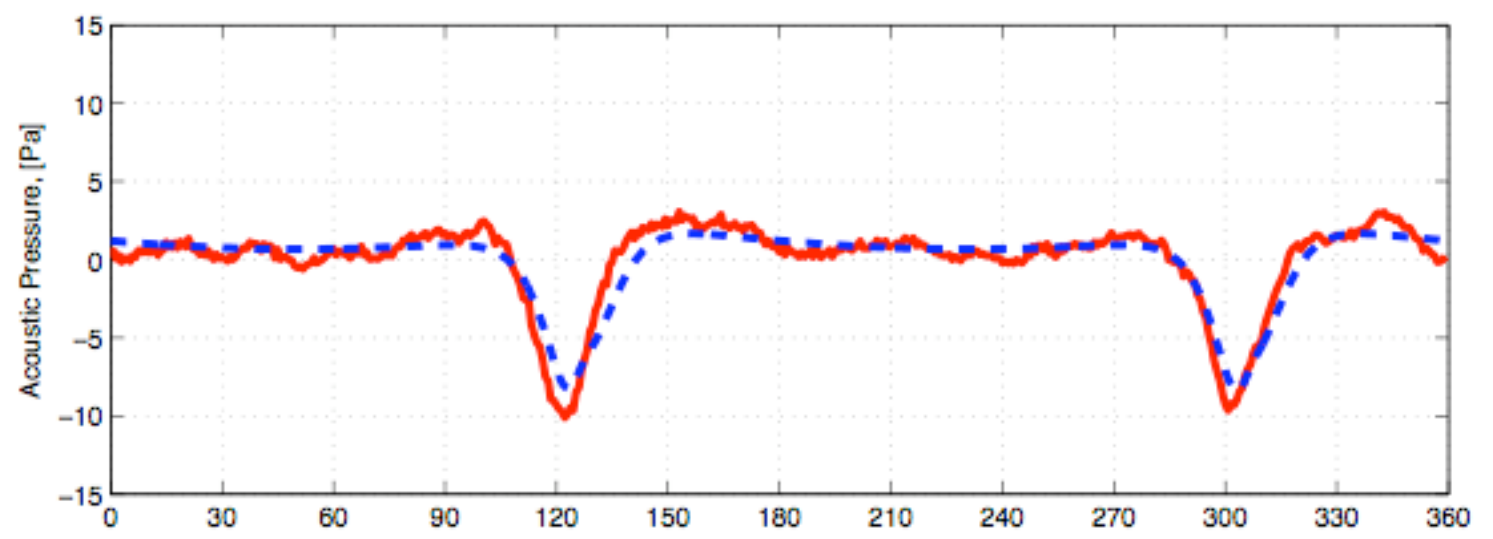

(d): $A 1(63 \mathrm{kts})$

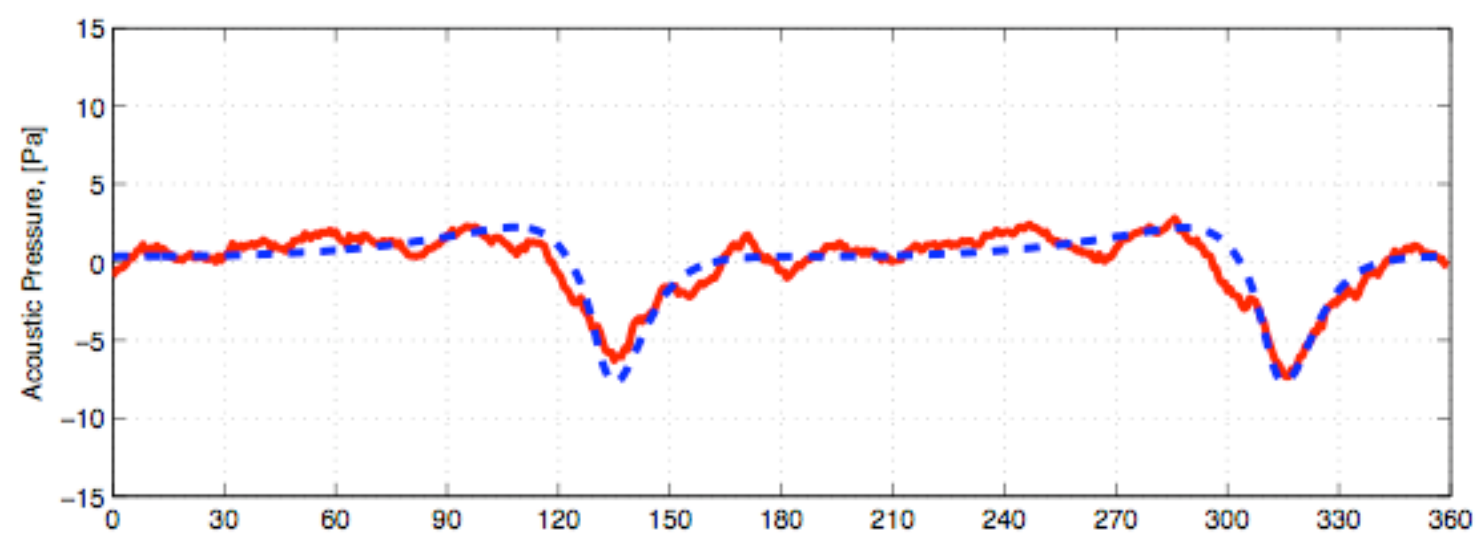

(e): $R 1(63 \mathrm{kts})$

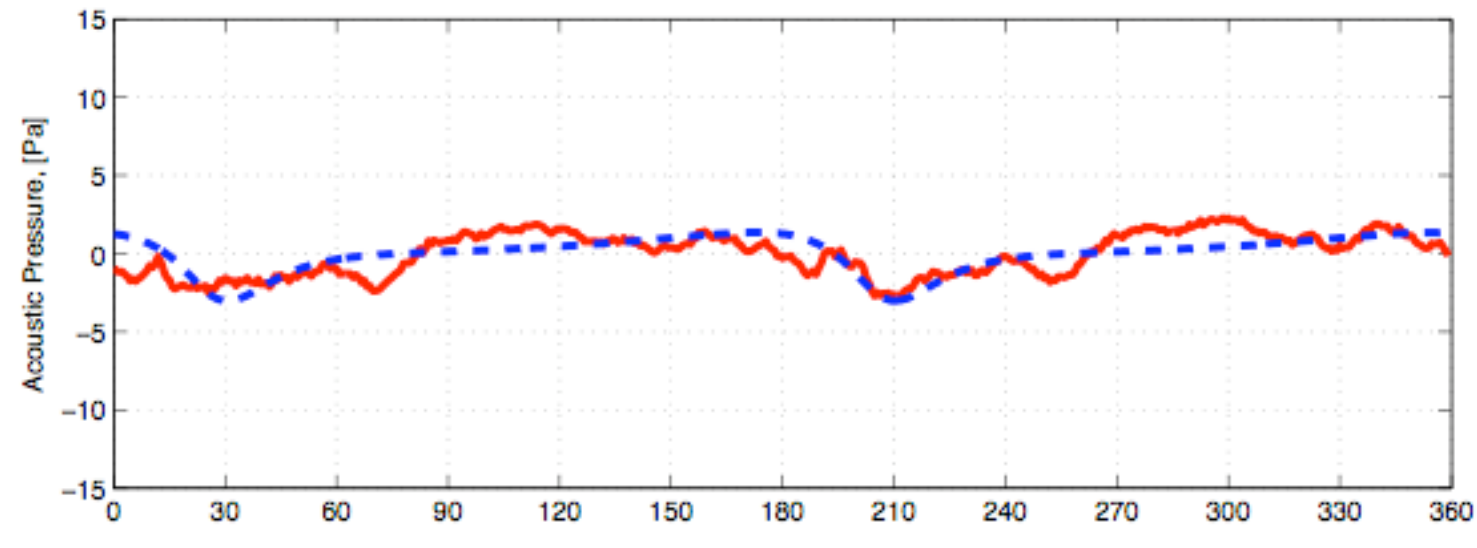

(f): $R 2(63 \mathrm{kts})$

$$
\text { = Experiment } \quad * \because=- \text { Theory }
$$

Figure 6.1: Theory and Experiment (Steady Level Flight, 63 knots) 
In order to investigate the larger differences between theory and experiment that occur most prominently at the outermost microphones (A4 \& R2), the sensitivity of the noise to changes in tail rotor air-loads was determined. In the past, the complicated nature of the aerodynamic environment in which the tail rotor operates has been thought to make the prediction of tail rotor air-loads and the resultant loading noise very difficult. Remembering that the tail rotor model used in the present research uses a uniform inflow assumption and was responsible for counteracting the entirety of the main rotor torque (in actuality a certain percentage of this torque would be counteracted by the lift generated by the vertical tailfin in forward flight), it is likely that the predicted thrust is substantially higher than in reality.

Figure 6.2 shows the sensitivity of the noise at microphone A4 (where a significant contribution to the noise comes from loading) to the predicted tail rotor thrust. Percentages are normalized with respect to the thrust predicted by simple momentum theory (See Section 5.3) and assuming that the tail rotor is responsible for counteracting $100 \%$ of the main rotor torque. The over-prediction of thrust is apparently accountable for much of the difference between theory and experiment, as Figure 6.2 (b) shows better peak-to-peak correlation with experiment for $75 \%$ of the originally determined tail rotor thrust. At $50 \%$ of the original tail rotor thrust, the theory slightly under predicts the measured peak levels. 


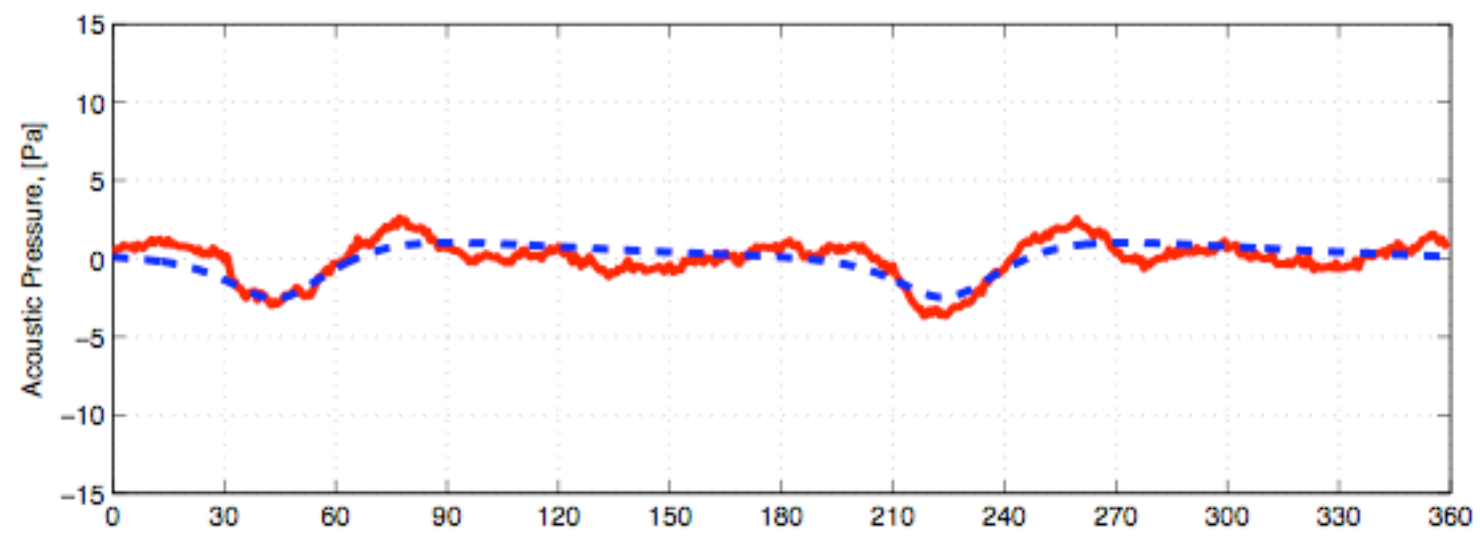

(a): A4 (63 kts) 100\% Predicted Tail Rotor Thrust

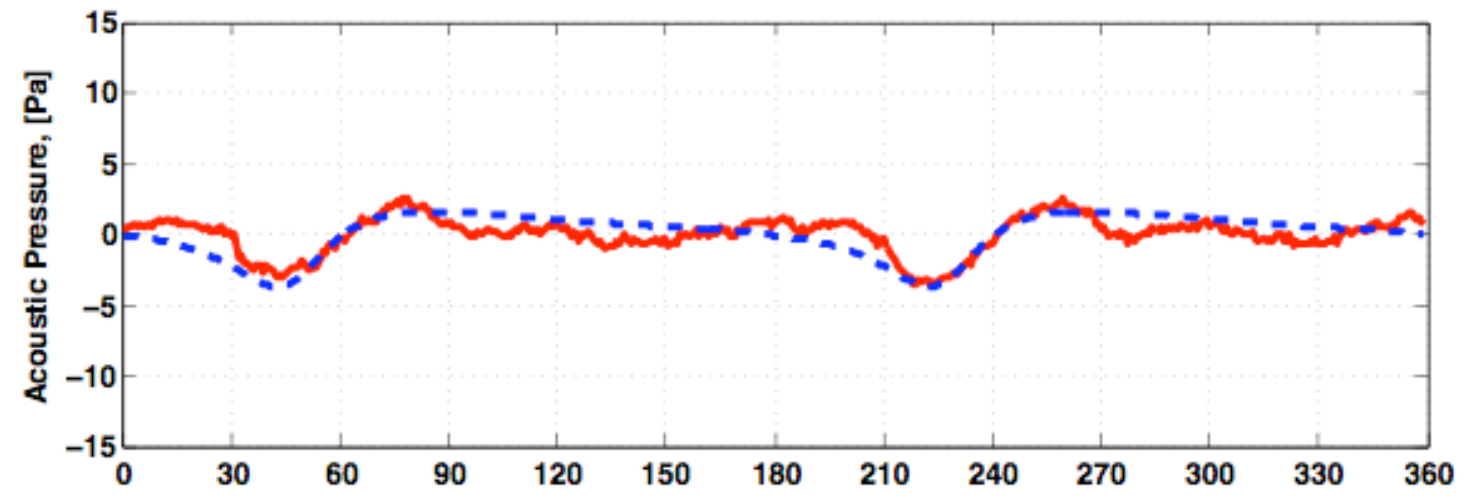

(b): A4 (63 kts) 75\% Predicted Tail Rotor Thrust

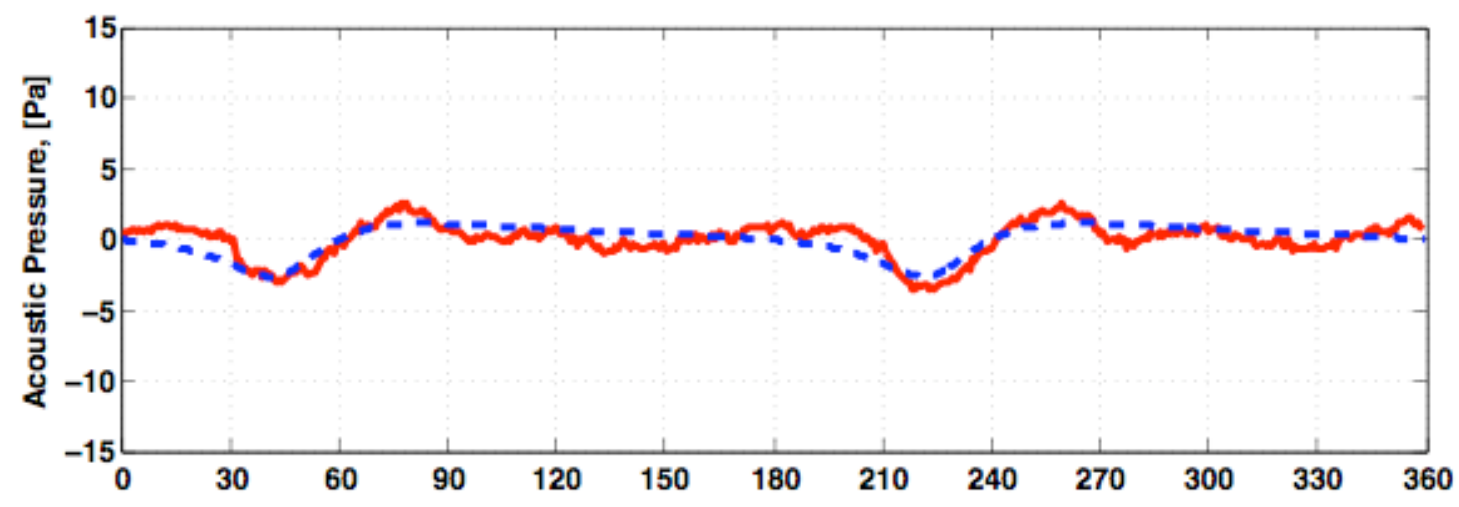

(c): A4 (63 kts) 50\% Predicted Tail Rotor Thrust

= Experiment $\quad= \pm= \pm=$ Theory

Figure 6.2: Sensitivity of Noise to Tail Rotor Thrust 


\subsection{Steady Descending Flight: Comparison with Theory}

In addition to comparing the theoretical results for steady level flight with the experimentally obtain time averaged, a comparison was also made using data obtained for the descending flight case. As expected, the comparison is still strong between theory and experiment.

Figure 6.3 shows the comparison at a selection of microphones for the -7 degree descending flight case. This is a case in which a significant amount of main rotor BVI occurs. As expected, both the pulse shape and amplitude are relatively well captured by the theoretical model, though the correlation is perhaps less strong at the microphones out-of-plane (where BVI is strongest). This again shows that to a large degree, the time histories are dominated by the classical thickness and loading noise.

As for the trends seen in the experimental data, and discussed in Section 4.4, the success of this comparison indicates that the reduction in OASPL as a function of increasing descent angle can be accounted for by the reduction in thrust that the tail rotor must produce as descent angle is increased. Though this certainly explains the reduction in pulse amplitude at the out of plane microphones, where loading noise is dominant, (See Figure 4.6) there was also a similar decrease in OASPL at the near in-plane microphones, where thickness noise was the dominant source. 


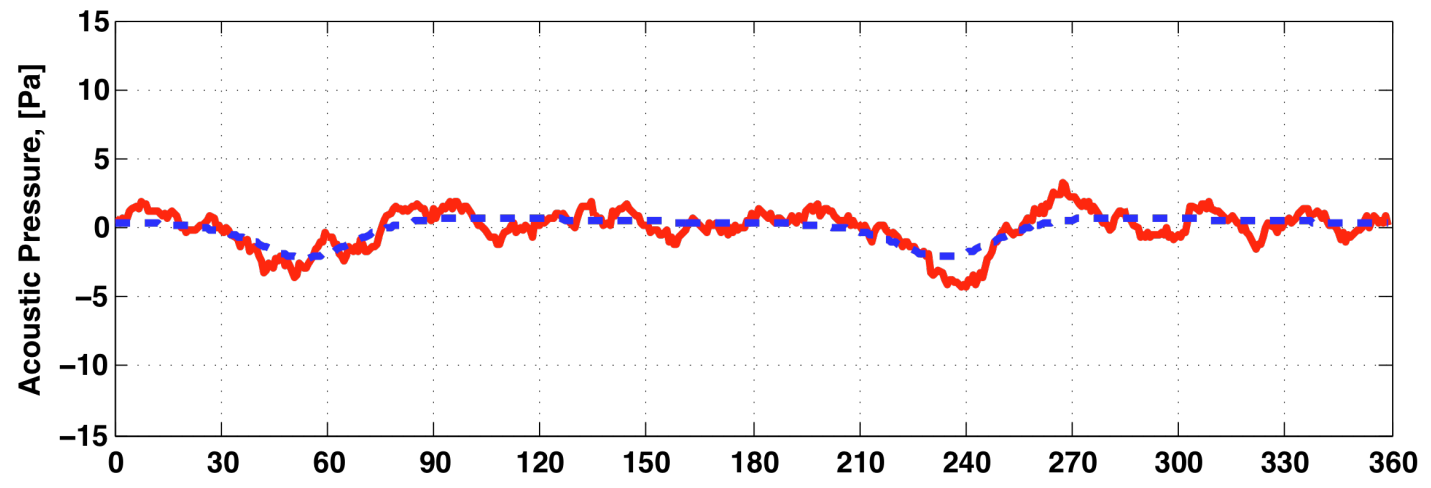

(a): $A 4$ (63 kts, -7 deg descent)

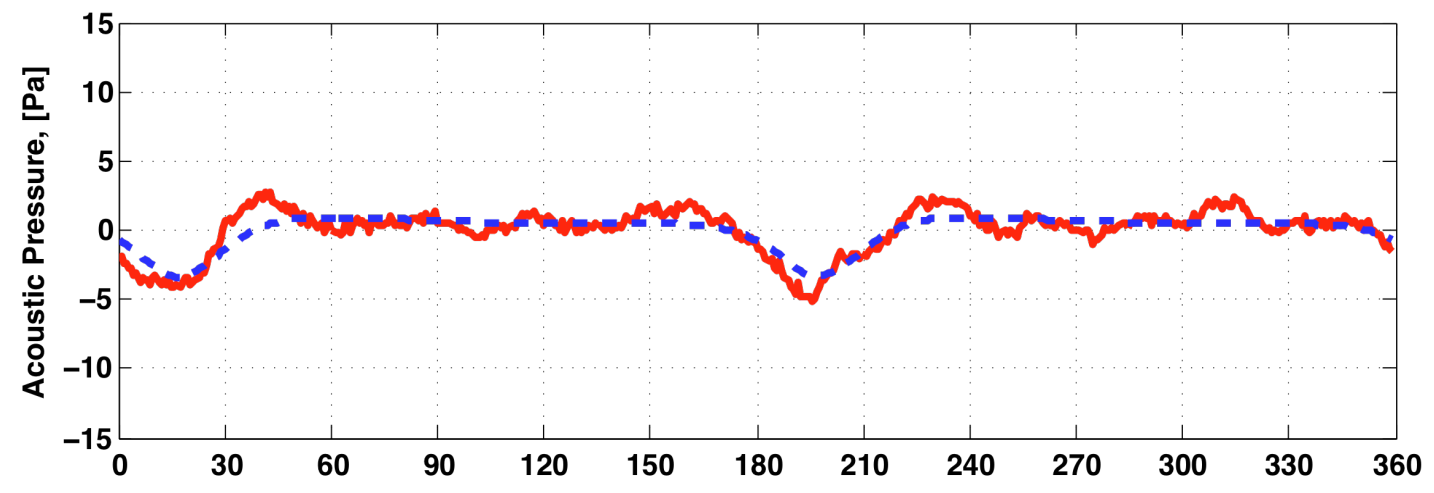

(b): $A 3$ (63 kts, -7 deg descent)

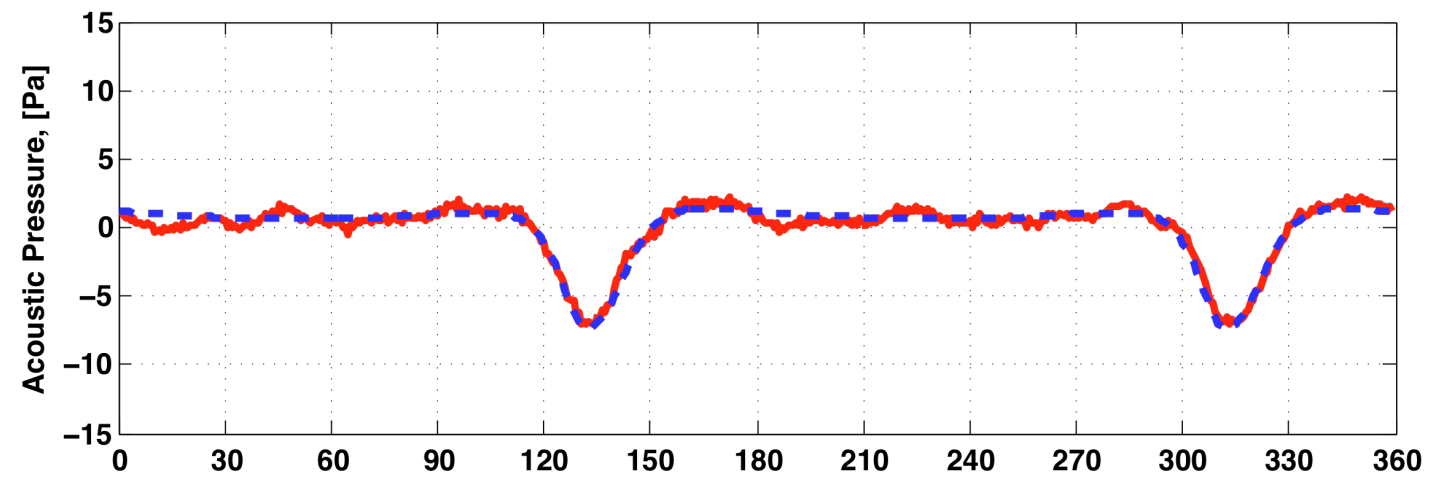

(c): $A 1$ (63 kts, -7 deg descent)

$$
\text { = Experiment } \quad \because \cdot \cdot=\text { = Theory }
$$

Figure 6.3: Theory and Experiment ( $63 \mathrm{kts},-7$ deg descent) 
In addition to the small reduction in amplitude associated with loading noise reduction, the reduction in thickness noise at the near in-plane microphones can also be accounted for. Though this might not be initially expected, closer inspection reveals that a descending flight condition results in a shift of the free-stream velocity. This effectively moves the location of the microphone with respect to the tail rotor azimuth location by $\gamma$, the glide slope angle. This physical shift is depicted in Figure 6.4. By rotating the position of the microphone with respect to the free-stream velocity, the phasing of the pulse will change, which, when combined with the phasing of the loading pulse, will reduce the amplitude of the pulse.

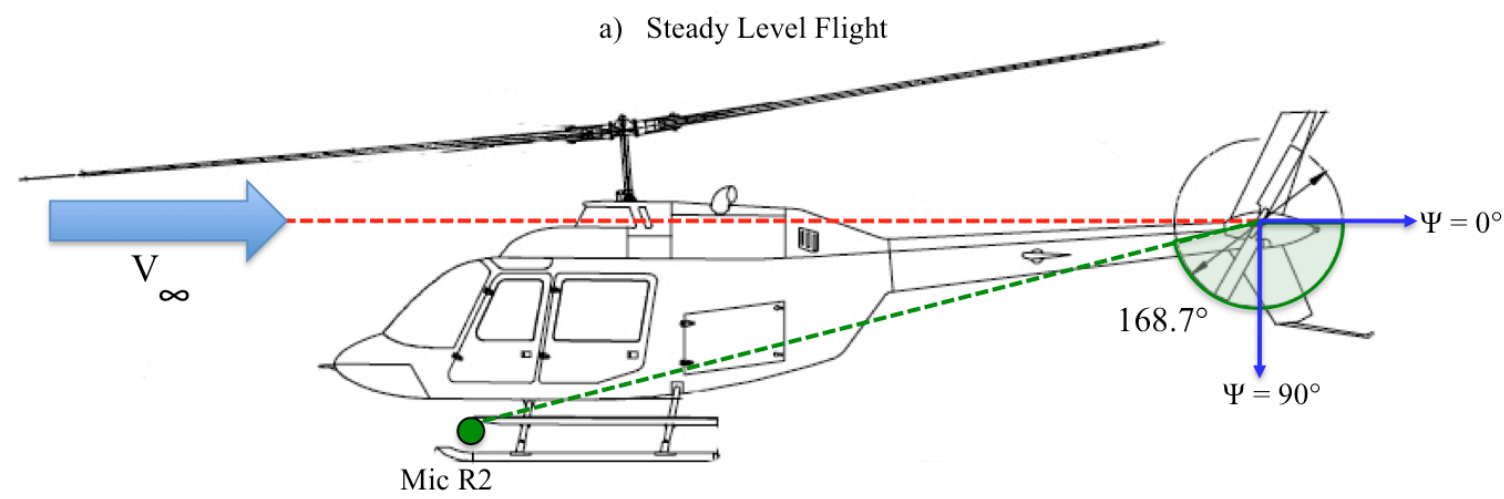

b) Steady Descent

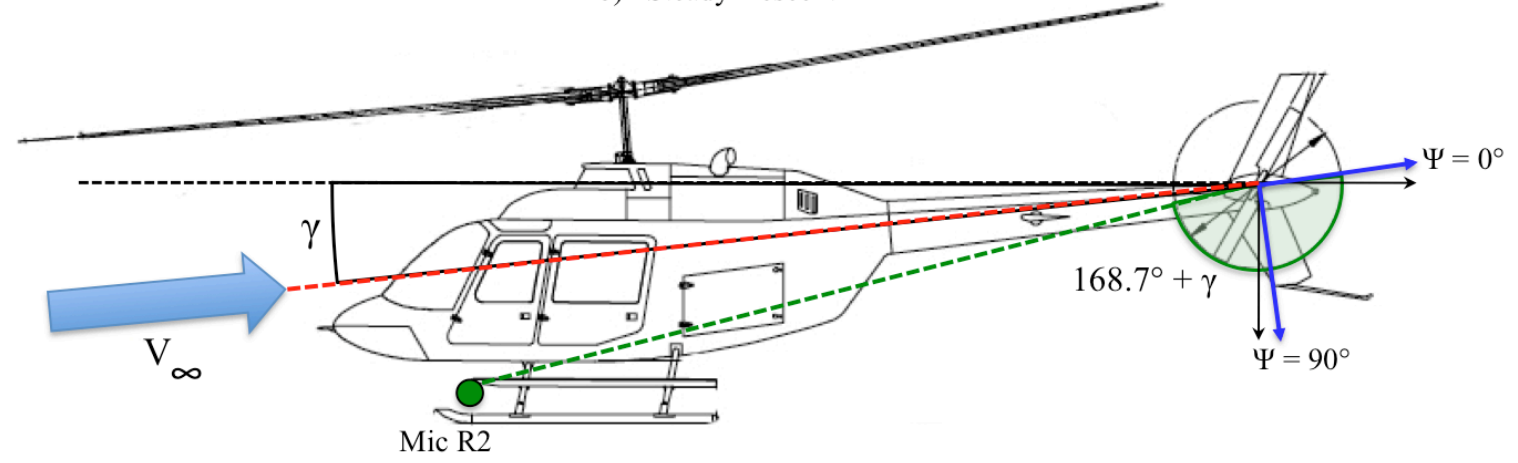

Figure 6.4: Effective change in microphone position as descent angle differs 


\section{Chapter $7 \quad$ Additional Considerations}

\subsection{Summary}

The comparison of the experimentally obtained time-averaged histories to the simple theoretical thickness and loading acoustic model of the 206B helicopter in Chapter 6 shows that, to a large extent, the time-averaged histories are dominated by thickness noise generated by the tail rotor for all near in-plane microphones, and by loading noise at the outermost microphones. The comparison with experiment is quite good and indicates that simple theoretical modeling of tail rotor acoustics can be quite useful in estimating the average harmonic noise of the Bell 206B helicopter tail rotor.

However, it is logical to ask if these average time histories are truly representative of the total noise radiated by the tail rotor under normal operating conditions; or if there are variation in the pulse time history that make the average value unrepresentative of the total noise being radiated by the helicopter's tail rotor. This issue is briefly addressed in this chapter by calculation of the standard deviation from this average value for the outof-plane microphones, A4, with the helicopter flying at 63 knots, in steady level flight. Though a conclusive statement concerning the existence or importance of any additional noise characteristics are beyond the scope of this work, this chapter aims to present the potential for future work in this area.

\subsection{Standard Deviation within Spectral Averages}

As discussed by Leverton et al, the detection of any interaction noise being produced by the tail rotor is an extremely complex process and requires a very precise narrowband 
frequency analysis. ${ }^{8}$ With the computing power available today, such an analysis is easily within reach.

One method of performing this analysis is to calculate the spectral average of 32 twosecond samples of acoustic data (corresponding to approximately 13 main rotor revolutions) in the frequency domain. In addition to the average of these spectra, the standard deviation was also computed. By examining the amplitude variation for all 32 spectra at an out-of-plane microphone, one can look for signs of any unsteady loading noise that might be as a direct result of an interaction between the tail rotor and main rotor wake, turbulence or other effects. Figure 7.1 shows this deviation for microphone A4, with the black line representing the spectral average, and the red and blue lines representing this average $\pm 2 \sigma$.

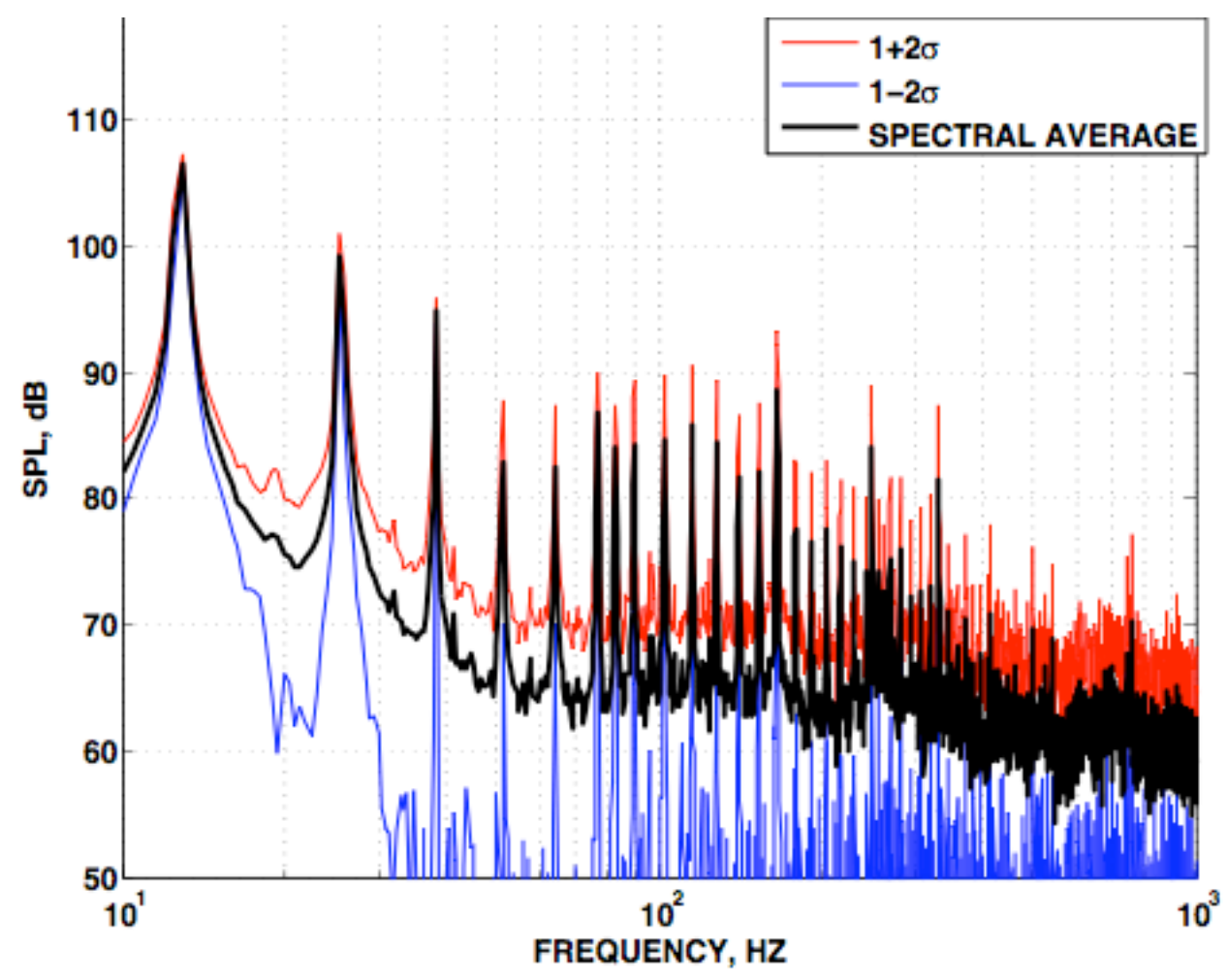

Figure 7.1: Spectral Average Measured on A4 $\pm 2 \sigma$ 
The $2 \sigma$ variations about the main rotor harmonics is seen to be quite small at the lower frequencies but grows markedly when the main rotor harmonic number increases. The variation in amplitude levels of higher harmonic noise suggests that other factors, such as atmospheric and/or wake effects or pilot induced unsteadiness, might be controlling the standard deviation at these higher frequencies. This method does not however account for any variations in rotor rotational frequency, which would also manifest itself as an increase in standard deviation at higher frequencies.

In order to separate the effects of frequency changes from actual physical changes in acoustic energy, the peak harmonic amplitudes of the first 600 main rotor harmonics $(\sim 7800 \mathrm{~Hz})$ and the first 94 tail rotor harmonics $(\sim 7800 \mathrm{~Hz})$ were computed for the 32 DFT's taken over the same time interval of thirteen main rotor revolutions $(\sim 2 \mathrm{sec})$. The standard deviations of these peak amplitudes were then calculated and plotted on a logarithmic axis system, shown in Figure 7.2 in which the red circles represent the amplitudes of the tail rotor harmonics and the main rotor harmonics are indicated by blue triangles. This method accounts for any variations in rotor rotational frequency from one spectrum to the next.

Figure 7.2 shows a significant amount of deviation for some of the harmonic noise amplitudes, but more importantly, it shows that this deviation still appears to be dependent on frequency, with the higher harmonic noise peak amplitudes displaying considerably higher deviations in SPL than the lower rotor harmonic noise peak amplitudes. It is probable that this frequency dependent increase in peak amplitude deviation is due in large part to signal jitter, a phenomenon that is intrinsically present in a spectral analysis of any rotor impulsive noise, even when measurements are taken in a 


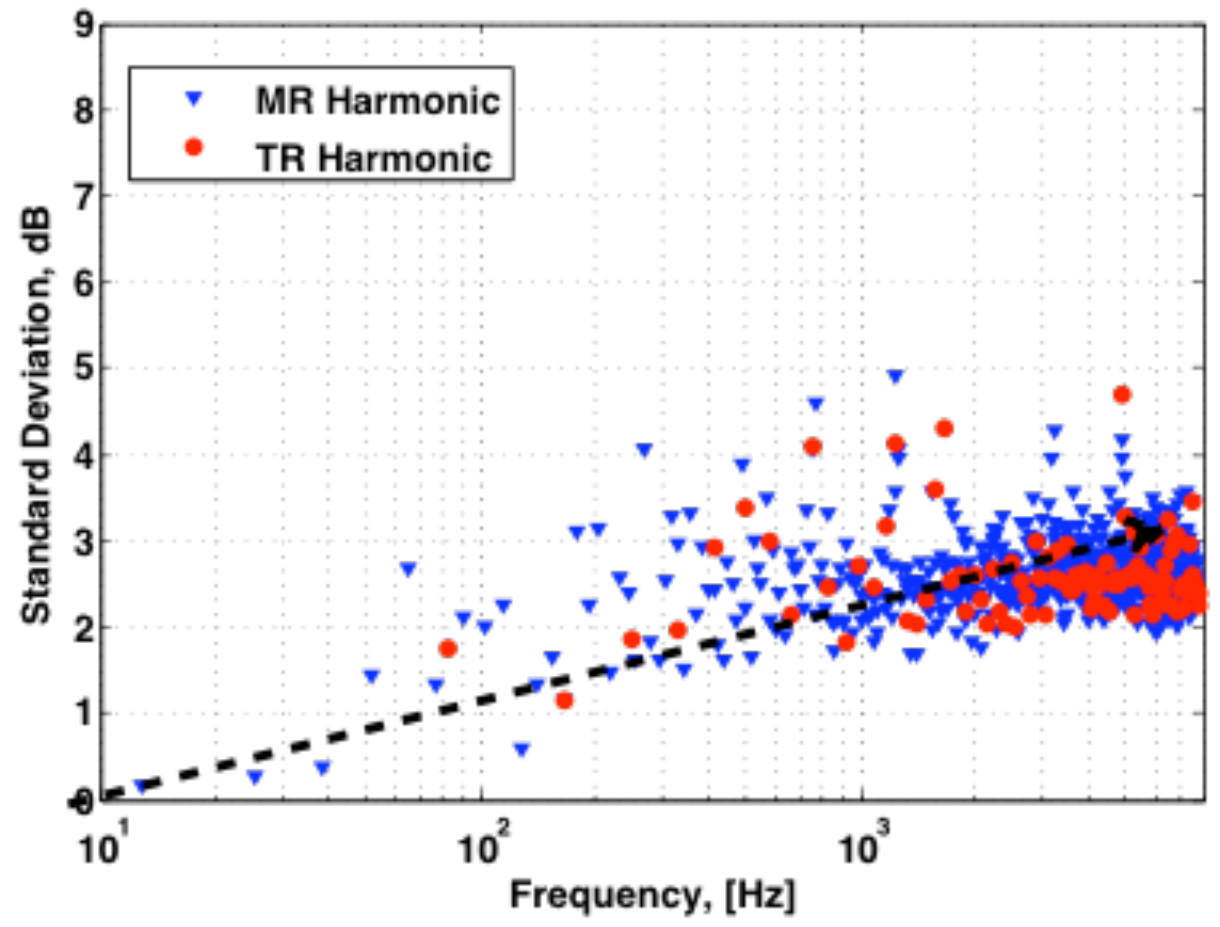

Figure 7.2: Standard Deviation of MR and TR Harmonic Amplitudes

wind-tunnel environment. Brooks explains this phenomenon in depth in reference 37 and shows that flight unsteadiness and other perturbations in the atmosphere, fuselage orientation, et cetera result in a spectral smearing that manifests itself as an increase in deviation as a function of frequency, much in the same fashion as shown in Figure 7.2. However, while signal jitter is likely responsible for a great deal of this deviation, especially at higher frequencies, this analysis does not rule out the possibility that other effects, such as main rotor/tail rotor interaction, fin effects, small local perturbations in the operating environment or sporadic tail rotor self-BVI, do not also exist. Recent work performed in the DNW have shown however, that these noise sources are generally small in comparison to the thickness and loading noise produced by the tail rotor ${ }^{41}$. Regardless, the deviations in this data likely represent a physical effect related to signal jitter and 
future prediction tools might be better served to incorporate some degree of signal jitter model into their prediction process.

\subsection{Further Thoughts}

Though a complete explanation of the mechanisms and sources of noise associated with all tail rotors is beyond the scope of this research, this chapter aimed to provide additional insight into this matter. Clearly, the time averaging process developed in the past several chapters does not provide a conclusive answer as to the importance of all of the various noise sources. It does however provide an extremely useful tool, which can be used alongside other methods (discussed in this chapter) to increase our understanding of such a complicated problem. The tail rotor, which operates in an extremely turbulent environment, is clearly not a noise source which can be easily understood using a single evaluation method or metric, but rather a combination of several. The existence of signal jitter, which can result in fluctuations as high as 5 or $6 \mathrm{~dB}$ shows that a more thorough investigation of this phenomenon should be conducted in the future so that its importance can be better understood. It also shows that this variability in harmonic noise is present in real world measurements and should be considered when estimating tail rotor noise levels. 


\section{Chapter $8 \quad$ Summary and Conclusions}

\subsection{Summary}

The main goal of this research was to develop a novel time domain averaging technique, using a theoretical tail rotor one-per-rev derived from a known main rotor one-per-rev and tail rotor/main rotor gear ratio, that could be used in conjunction with a flying array of microphones attached to a full-scale helicopter to isolate periodic tail rotor noise during various flight conditions. Results obtain using this technique were then used in conjunction with a discrete Fourier analysis to shed light on the mechanisms and directivity of the tail rotor of the Bell 206B helicopter. Though many of the conclusions drawn throughout this work pertain specifically to this helicopter, this research provides the information needed to use this time averaging technique on any helicopter outfitted with the appropriate instrumentation.

In addition to describing the time averaging technique, this thesis also compared the periodic time averages with classical thickness and loading noise acoustic theory, developed from first principles and a simple tail rotor blade-loading model. This comparison resulted in an understanding that a great deal of the periodic noise generated by the tail rotor can be attributed to both the thickness and operational tip Mach number of the blade as well as the loading undergone by the rotor. This knowledge could prove to be valuable in future tail rotor designs.

Finally, some of the pitfalls of using a time averaging technique were examined, specifically dealing with the detection of noise due to the interaction between the main 
rotor wake and tail rotor. The goal of this discussion was to show the reader that the complicated nature of the tail rotor necessitates the use of not any one single analysis technique but rather a conglomeration of several. A frequency analysis of the deviations in SPL showed that there was an increase in harmonic noise peak amplitude deviation that was dependent on the frequency of the noise. This indicated that signal jitter is important when considering the total noise emitted from the tail rotor and that this phenomenon should be examined more closely in the future.

\subsection{Conclusions}

In this section the major conclusions that have been made for this thesis are described:

1. Time domain averaging using a theoretical tail rotor one-per-rev derived from a known main rotor one-per-rev and tail rotor/main rotor gear ratio used together with a flying array of microphones attached to a helicopter, has been shown to be a useful method of isolating periodic tail rotor noise.

2. For the Bell 206B helicopter, it has been shown that periodic tail rotor harmonic noise is dominated by thickness noise during both level and descending flight near the plane of the tail rotor (and thus near the fuselage), and that there is a strong contribution of loading noise at the out-of-plane microphone positions. Additional contributions from other aperiodic source of noise, though existent, were concluded to be secondary in importance with respect to the harmonic thickness and loading noise generated by the rotor. From a practical point of view, this means that reducing the thickness of the rotor and reducing the tip Mach number will result in significant reductions in tail rotor noise for this helicopter. 
3. The contribution of loading noise to the acoustic pulse shape was shown to reduce during descending flight, when the tail rotor produces less thrust to counter the main rotor torque. Furthermore, it was shown that the OASPL increases approximately $3 \mathrm{~dB}$ as forward speed increases from 38 to 70 knots, and that it decreases approximately $2 \mathrm{~dB}$ as helicopter descent angle is increased from 0 to 12 degrees.

4. Classical thickness and loading noise acoustic theory, developed from first principles and a simple tail rotor blade loading model, predicts the pulse shape and amplitude of the time history for most microphone positions quite well. This supports previous conclusions from wind tunnel testing stating that isolated thickness and loading noise effects are the dominant source of averaged periodic tail rotor harmonic noise.

5. By examining the standard deviation from the averaged tail rotor noise in the frequency domain, fairly large deviations were found. In order to separate the affect of small frequency shifts from changes in amplitude SPL, the amplitudes were found at the corresponding harmonic frequencies of both the main and tail rotors. The deviation in this amplitude was very low up to a few hundred $\mathrm{Hz}$, but increases as frequency increases. This growth is indicative of the existence of "signal jitter" which often exists in the measurements of rotor impulsive noise. The deviations may also indicate the existence of aperiodic acoustic energy due to atmospheric turbulence, flight unsteadiness, or turbulence within the tail rotors operating environment, however it does not definitively indicate the existence of noise generated by the main rotor wake/tail rotor interaction. 


\subsection{Recommendations for Future Work}

The results and techniques presented in this thesis should provide valuable tools for the future study of tail rotor noise. However, until the noise levels associated with the tail rotor have reached levels well below the main rotor, research in this area will continue to grow. The time averaging technique developed in this research will certainly prove to be a valuable tool for future research. Below are some suggested further areas of study.

- Additional Flight Conditions: This thesis focused primarily on the development of the time averaging method and only focused on two flight conditions, level and descending flight. However, during the 2006 flight test, some data was also taken for conditions that included ascending flight, turning flight and decelerating flight. Because of the importance that the relationship between the main rotor wake and tail rotor wake can have, it is possible that some of these flight conditions might show stronger signs of additional aperiodic noise (main rotor wake/tail rotor interaction). Though some flight conditions might have to be rerun in an additional flight test, the data obtained in 2006 provides a unique opportunity for a thorough examination of the Bell 206B tail rotor during a plethora of flight conditions.

- Additional Tail Rotor Configurations: The tail rotor of the Bell 206B is a relatively small, pusher type, advancing side down tail rotor. Many past studies have concluded that these different parameters can play a large role not only in the periodic noise (thickness and loading) associated with the rotor but also the existence of additional noise (main rotor wake/tail rotor interaction, Self BVI, HSI, etc.). One interesting possibility for future research would be to conduct an 
experiment similar to the 2006 CRI flight test using a different helicopter with a different tail rotor configuration. This technique could be also be used to study a fenestron (Fan-in-fin) tail rotor. Ultimately, this technique provides a valuable tool with which a database of tail rotor time histories can be compiled. This database could then be used for comparison with any theoretical or computational work done in the future.

- Improvement of the Tail Rotor Loading Model: The loading model developed in this work was very simple and though it still served to provide a reasonable comparison with the experimentally obtained averages, it could certainly be improved upon. The assumption of uniform inflow could be further developed, as the operating environment in which the tail rotor actually operates is far from uniform. In order to accomplish this, a thorough investigation of the fuselage, main rotor, hub and engine wake would need to be conducted in conjunction with an in-depth aero-acoustic calculation of the blade loads. This has been attempted in the past, though much of the work was done without any experimental data for comparison. The time averaging technique from this research provides the needed data. In addition to the inflow assumption, the time averages obtained experimentally showed signs of some higher harmonic loading noise, most likely due to the aerodynamic interaction between the vertical stabilizer and tail rotor. The incorporation of these effects into the loading model would shed light on whether these higher harmonic phenomena were in fact associated with the vertical fin. Furthermore, the use of in-flight blade pressure transducers would provide a means with which to validate the aerodynamic prediction scheme. 
- Model Testing: Because of lack of comparable tail rotor theoretical noise modeling, the theoretical tail rotor model had little else that it could be compared to or validated with beyond the experimentally obtained time averages. It could prove useful to conduct a simple model test (at near full-scale, in isolation) in the University of Maryland Acoustic chamber ${ }^{5}$ or a similar environment. Doing so would provide a proven experimental venue in which data could be generated and compared to the theoretical and experimentally obtained time averages. Additional work could also be done using this chamber to investigate the affect that an orthogonal vortex interaction (such as that which would occur when the main rotor wake interacted with the tail rotor) has on the total noise profile of the tail rotor.

- Improvements to the 2006 Flight Test: Though the majority of the problems associated with this flight test could clearly be worked around, any future flight tests should be sure to make a few improvements.

- The generation of the tail rotor one-per-rev required the estimation of the phasing of the rotors with respect to each other. By using an actual tail rotor one-per-rev sensor, as in the 2008 flight test, this process could be avoided and there would be an improvement in time averaged signal quality.

○ Because the 2006 Flight Test was originally conducted to examine main rotor BVI, four microphones were used on the advancing side of the main rotor and two were used on the retreating side. It would be beneficial to 
use more microphones (especially near the plane of the tail rotor) to get a better view at the directivity pattern of the tail rotor.

- The University of Maryland Microphone boom array greatly limited the maximum forward speed that the Bell 206B could reach. The use of a faster more powerful helicopter would allow for an investigation of the tail rotor noise at faster speeds. 


\section{APPENDIX A - Brüel and Kjær Microphone Data Sheet}

For the 2006 Flight test, two different types of microphones were used. Microphones A3 and A4 were $1 / 4$ inch free-field B \& K microphones (model \# 4939) with a dynamic range of 28 to $165 \mathrm{~dB}$, a sensitivity of $4 \mathrm{mV} / \mathrm{Pa}$ and a frequency range of $4-100 \mathrm{kHz}$. The frequency response of this microphone (without a nose cone) is shown below in Figure A.1. More information regarding this microphone can be found in reference 38 .

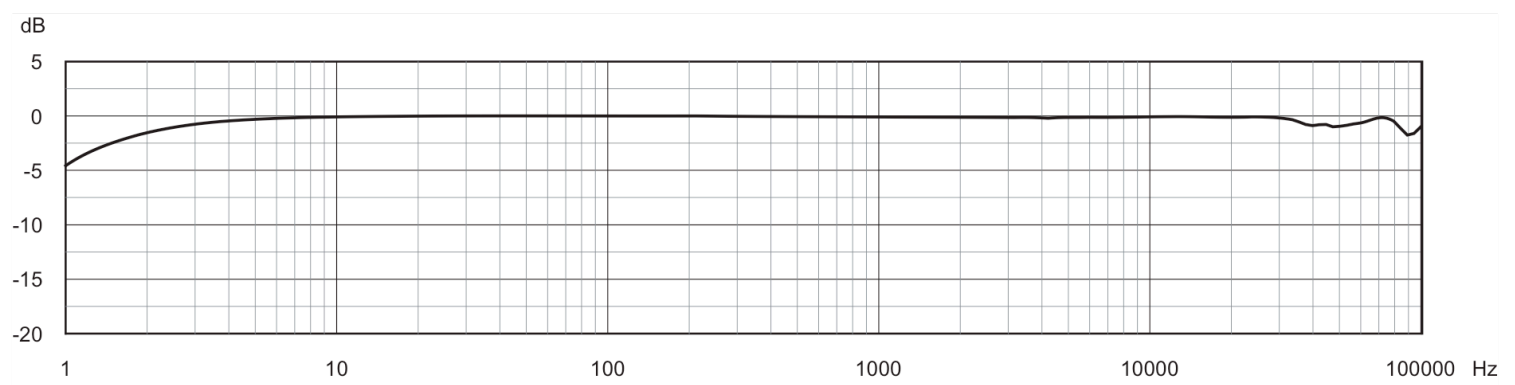

Figure A.1: Frequency Response of 4939 microphone (No Nose Cone)

Microphones A1, A2, R1 and R2 used instead a $1 / 2$ inch free-frield B \& K microphone (model \# 4191) with a dynamic range of 21.4 to $161 \mathrm{~dB}$, a sensitivity of $12.5 \mathrm{mV} / \mathrm{Pa}$, and a frequency range of $3.15-40 \mathrm{kHz}$. The frequency response of this microphone (without a nose cone) is shown below in Figure A.2. More information regarding this microphone can be found in reference 39 .

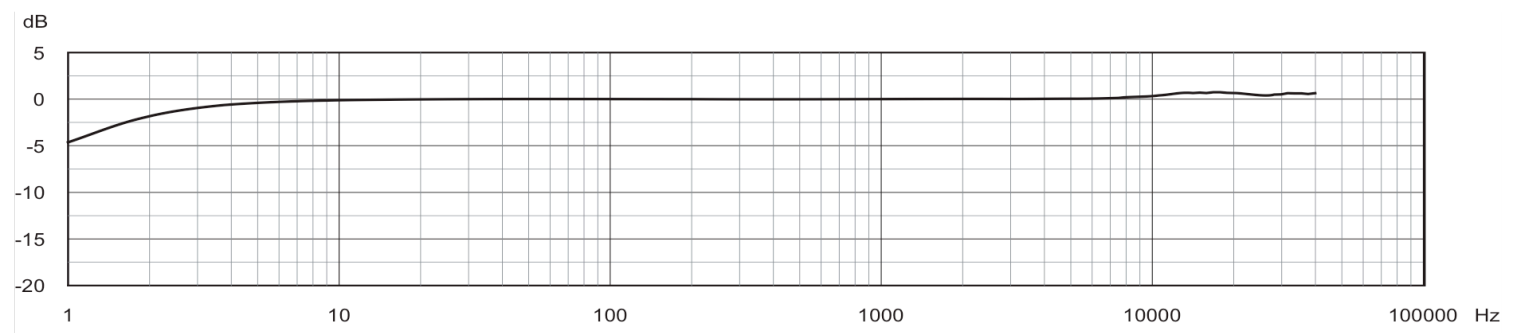

Figure A.2: Frequency Response of 4191 microphone (No Nose Cone) 
In addition to the microphones, nose cones were used on all six microphones. The use of these nose cones substantially reduces the wind noise at high speed. The nose cone, as well as the frequency response of the $1 / 2$ inch microphone at a $0^{\circ}$ incidence angle are shown below in Figure A.3. Figure A.4 shows the dependence of this response on the incidence angle of the microphone. Clearly the nose cone only influences very high frequencies (higher than $10 \mathrm{kHz}$ ). These frequencies are well above those of interest in this research. More information concerning the nose cone can be found at 40 .
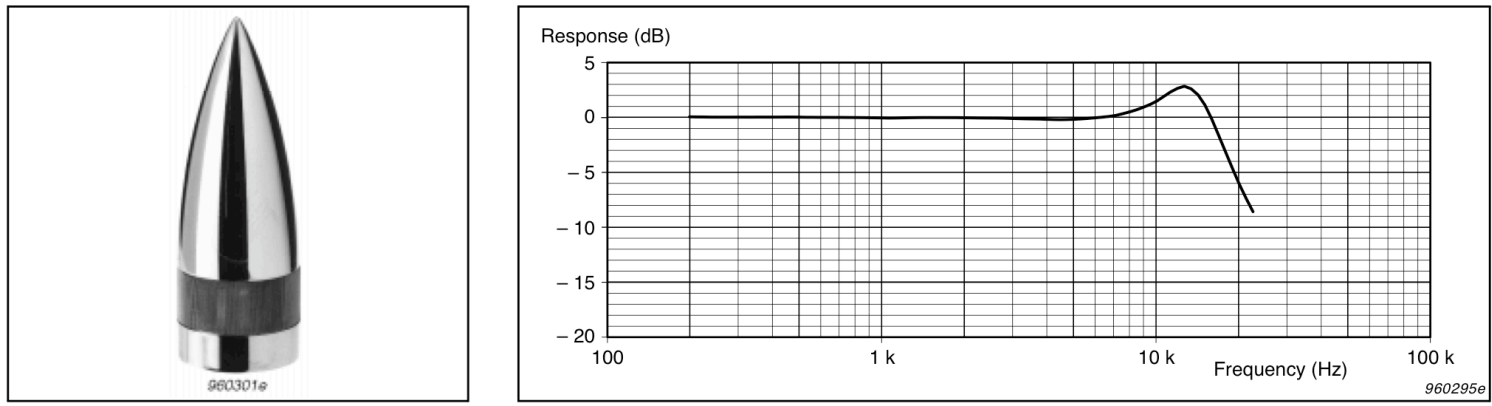

Figure A.3: Mic. nose cone and frequency response of $1 / 2$ in. microphone at 0 deg.

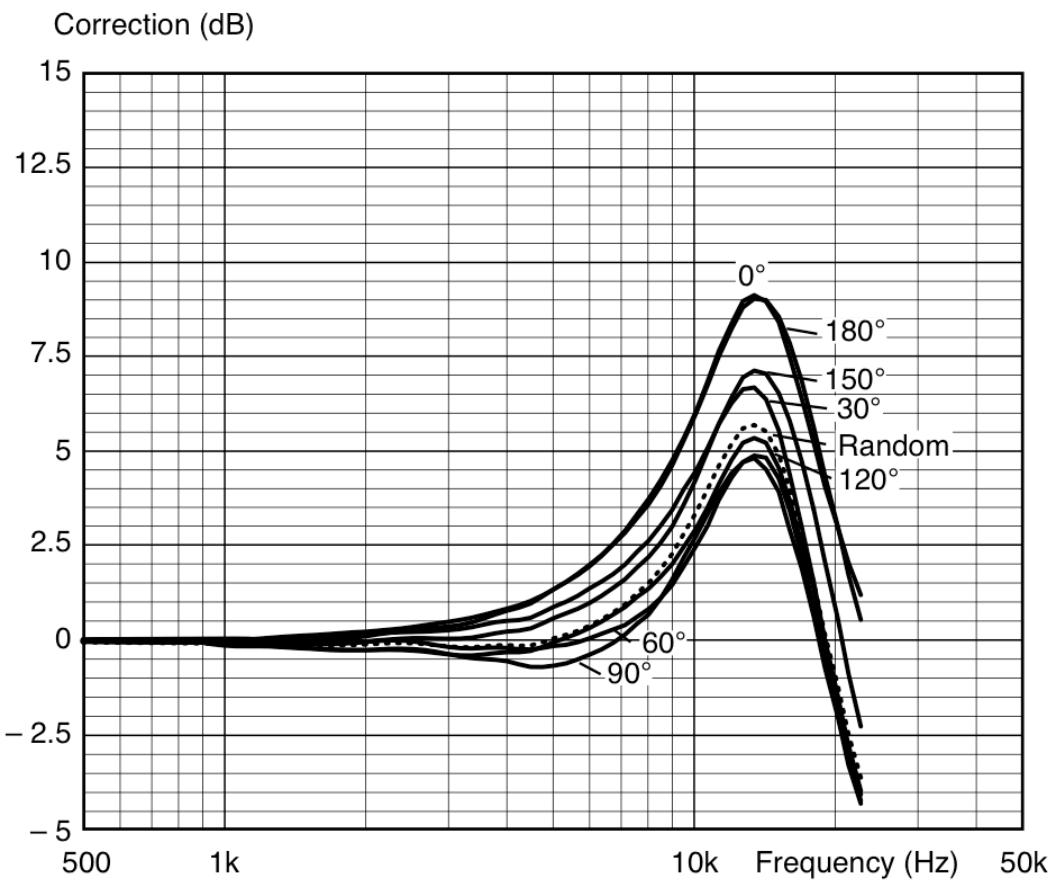

Figure A.4: Free-field correction curve as a function of incidence angle 


\section{APPENDIX B - Tail Rotor Model Validation}

In order to validate the trim equation for the tail rotor, a comparison was made with the closed form of the solution for the tail rotor trim found on page 187 of reference 36 . Keeping in mind that Prouty uses different definitions of flapping, pitch-flap coupling angles and inflow in his closed form solutions, the plots in Figure B.1, Figure B.2, and Figure 5.7 show the equivalent trim solutions obtained using Prouty's equations alongside those used in this research (red markers) for several values of pitch-flap coupling. The trim solution used by Prouty assumes a simple linear inflow, so the program used in this research was adjusted to include the same linear inflow. Figure B.1, Figure B.2, and Figure 5.7 serve only to show that the tail rotor trim solution used in this research has been validated with Prouty's closed form solution.

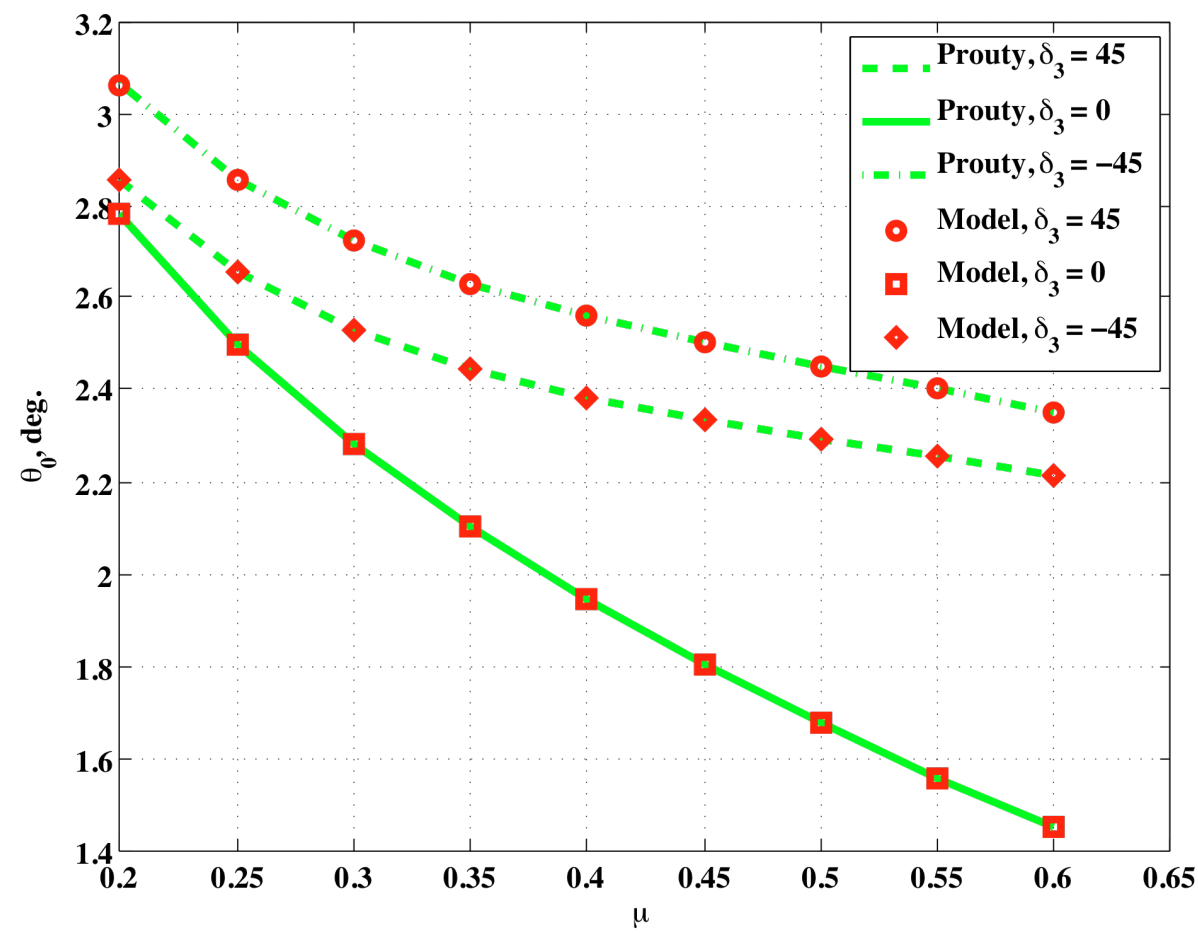

Figure B.1: Trim Validation (Collective) 


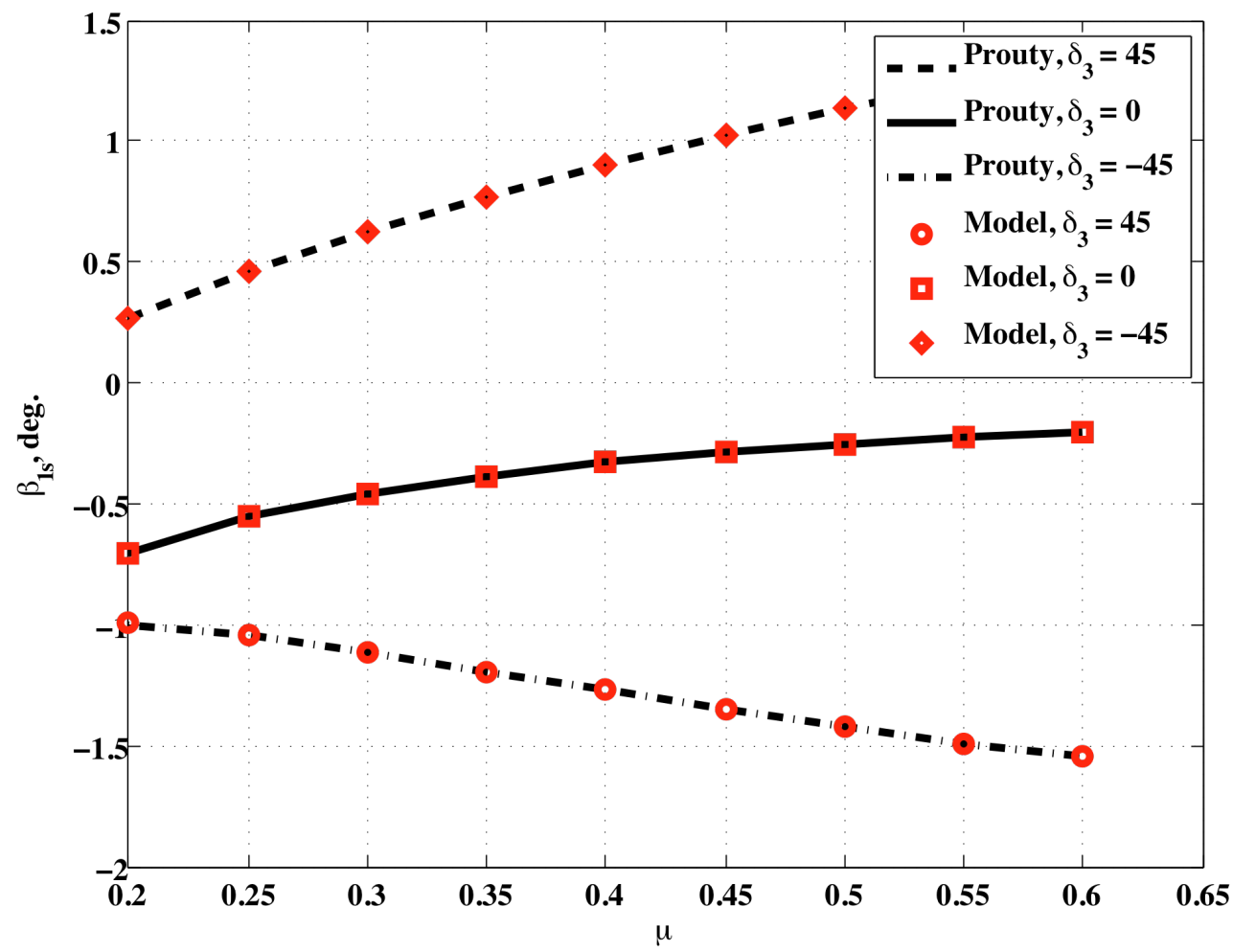

Figure B.2: Trim Validation (Lateral Flapping)

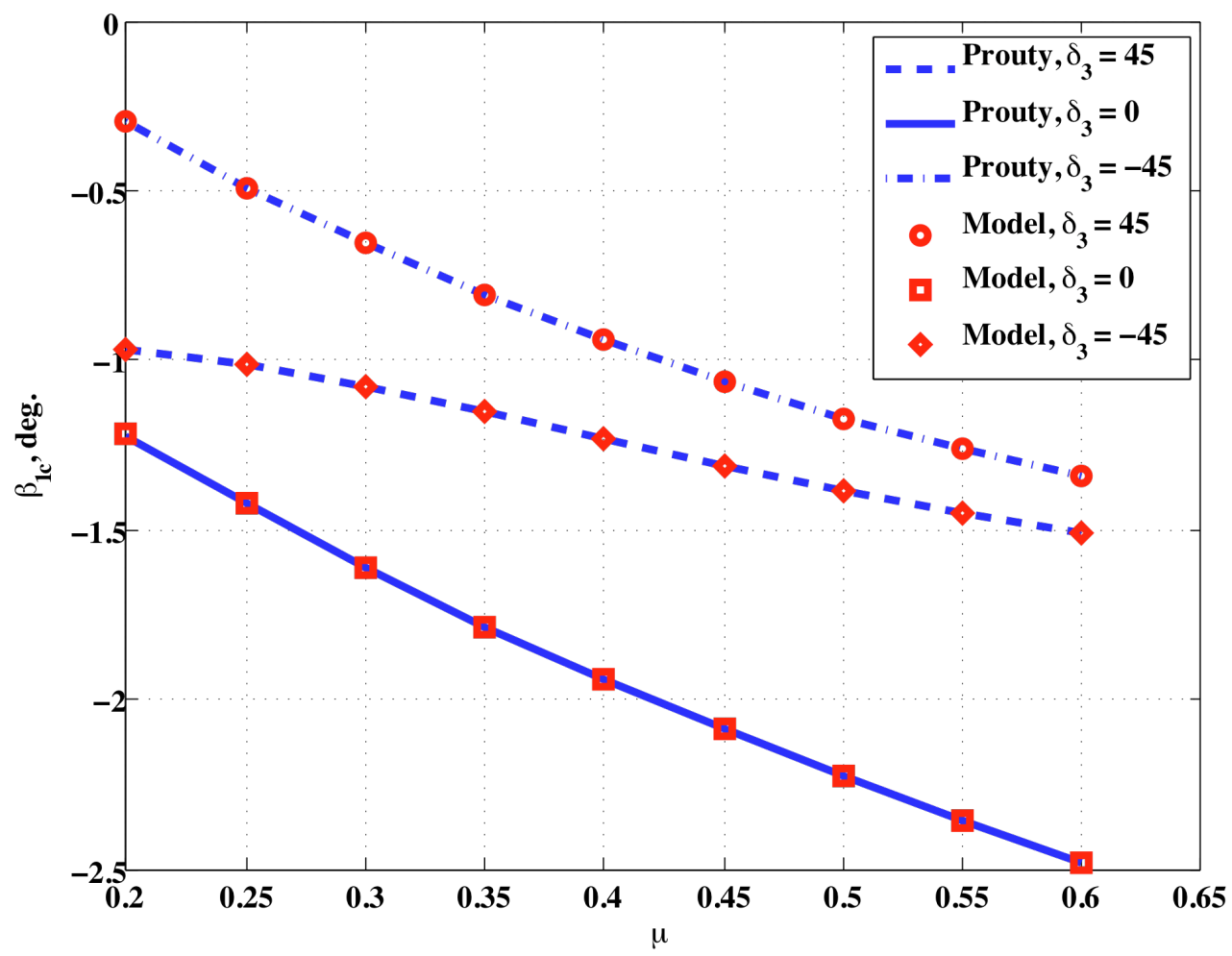

Figure B.3: Trim Validation (Longitudinal Flapping) 


\section{References}

1. Chou, S.T., "A Study of Rotor Broadband Noise Mechanisms and Helicopter Tail Rotor Noise”, NASA CR 177565, August 1990.

2. Powell, C. and Fields, J., "Human Response to Aircraft Noise", Aeroacoustics of Flight Vehicles: Theory and Practice, Vol. 2: Noise Control, August 1991.

3. Bishop, D.E, Simpson, M.A, Beranek, B., Chang, D., "Experimental Atmospheric Absorption Values from Aircraft Flyover Noise Signals", NASA CR-1751, June 1971.

4. Schmitz, F.H., and Yu, Y.H., "Helicopter Impulsive Noise: Theoretical and Experimental Status,” Journal of Sound and Vibration, Vol. 109,No. 3, 1986.

5. Koushik, S.N., “A New Experimental Approach to Study Helicopter Blade-Vortex Interaction Noise.” Ph.D. diss., University of Maryland, 2007.

6. Lynn, R.R., Robinson, F.D., Batra, N.N., and Duhon, J.M., “Tail Rotor Design Part 1: Aerodynamics", Journal of the American Helicopter Society, Vol. 15, No. 4 October 1970.

7. Hirsh, N.B. and Ferris, H.W., "Design Requirements for a Quiet Helicopter," Proceedings of the $28^{\text {th }}$ Annual American Helicopter Society Forum, No. 600, 1972.

8. Leverton, J.W., and Pike, T.C., "The Importance of Tail Rotor Interaction as an Acoustic Source", American Helicopter Society $49^{\text {th }}$ Annual Forum, St. Louis, MO, 1993.

9. Robinson, F. "Component Noise Variables of a Light Observation Helicopter", NASA CR-114761, January 1973. 
10. Barlow, W.H., McCluskey, W.C., Ferris, H.W., “OH-6A Phase II Quiet Helicopter Program", USAAMRDL Technical Report 72-29, September 1972.

11. Leverton, J.W., Pollard, J.S., and Willis, C.R., "Main Rotor Wake/Tail Rotor Interaction", Vertica 1, 213-221, 1977.

12. Pegg, J.P., and Shidler, P.A., "Exploratory Wind-Tunnel Investigation of the Effect of the Main Rotor Wake on Tail Rotor Noise", NASA Helicopter Acoustics, Aug 1, 1978.

13. Chou, S.T., George, A. "Progress in Tail Rotor Noise Analysis", American Helicopter Society $42^{\text {nd }}$ Annual Forum, Washingon, DC, June 1986, Vol 1.

14. Fitzgerald J. and Kohlhepp, F., "Research Investigation of Helicopter Main Rotor/Tail Rotor Interaction Noise”, NASA CR 4143 May 1988.

15. Martin, R.M., Burley, C.L., and Elliott, J.W., "Acoustic Test of a Model Rotor and Tail Rotor: Results for the Isolated Rotors and Combined Configuration”, NASA TM 101550, February 1989.

16. Schultz, K.J. and Splettstoesser, "Helicopter Main Rotor / Tail Rotor Noise Radiation Characteristics from Scaled Model Rotor Experiments in the DNW", American Helicopter Society $49^{\text {th }}$ Annual Forum, St. Louis, MO, May 1993

17. Tadhighi, H., "An Analytical Model for Prediction of Main Rotor/Tail Rotor Interaction Noise," AIAA $12^{\text {th }}$ Aeroacoustics Conference, San Antonio, TX, April 1989.

18. Sim, B.W., Beasman, T., Schmitz, F.H., and Gopalan, G., "In-Flight Blade Vortex Interaction (BVI) Noise Measurements Using a Boom-Mounted Microphone Array", American Helicopter Society $60^{\text {th }}$ Annual Forum, Baltimore, MD, 2004. 
19. Schmitz, F.H., Greenwood, E., Sickenberger, R., Gopalan, G., Sim, B.W., Connor, D., Moralez III, E. and Decker, W.A., "Measurement and Characterization of Helicopter Noise in Steady-State and Maneuvering Flight", American Helicopter Society $63^{\text {rd }}$ Annual Forum, Virginia Beach, VA, May 2007.

20. http://www.bellhelicopter.com/en/aircraft/commercial/pdf/B3_2006_jan_web.pdf Bell 206B3 Product Specification, January 2006.

21. Moralez, Ernesto III, Tucker, George E., Hindson, William S., Frost, Chad, R., and Hardy, Gordon H., "In-Flight Assessment of a Pursuit Guidance Display Format for Manually Flown Precision Instrument Approaches," Proceedings of the American Helicopter Society $60^{\text {th }}$ Annual Forum, Baltimore, MD. June 2004.

22. Sim, B.W., and Schmitz, F.H., "Acoustic Phasing and Amplification Effects of Single Rotor Helicopter Blade-Vortex Interactions," American Helicopter Society $55^{\text {th }}$ Annual Forum, Montreal, Canada, May 1998.

23. Cook, C.V., "A Review of Tail Rotor Design and Performance", Vertica 2, pp.163$181,1978$.

24. Schmitz, F.H., and Boxwell, D.A., "In-Flight Far-Field Measurement of Helicopter Impulsive Noise", American Helicopter Society $32^{\text {nd }}$ Annual Forum, 1976.

25. Boxwell, D.A., and Schmitz, F.H., "Full-Scale Measurements of Blade-Vortex Interaction", American Helicopter Society $36^{\text {th }}$ Annual Forum, 1980.

26. Cross, J.L., and Watts, M.E., "In-Flight Acoustic Testing Techniques Using the YO3A Acoustic Research Aircraft,” NASA TM-85895, February 1984. 
27. Ishii, H., Gomi, H., and Okuno, Y., "Helicopter Flight Tests for BVI Noise Measurement Using an Onboard External Microphone,” AIAA Atmospheric Flight Mechanics Conference, AIAA 2005-6119, August 2005, San Fransisco, CA.

28. Lee, S., Erwin, J.P., and Brentner, K.S., "Acoustic Scattering of Rotorcraft Noise”, American Helicopter Society $64^{\text {th }}$ Annual Forum, Montreal, Canada, May 2008.

29. Lyrintzis, A.S., Koutsavdis, E. and Strawn, R.C., "Technical Note: A Comparison of Computational Aeroacoustic Prediction Methods", Journal of the American Helicopter Society, Vol. 42, No. 1, pp. 54-57, 1997.

30. Juve, D., "Aeroacoustics: From Fluid Dynamics to Acoustics", von Karman Institute for Fluid Dynamics Lecture Series 1997-07, Sept. 15-18, 1997.

31. Ffowcs Williams, J.E. and Hawkings, D.L, "Sound Generation by Turbulence and Surfaces in Arbitrary Motion," Philosophical Transactions of the Royal Society of London, A264, pp. 321-342, 1969.

32. Gopalan, G., “Quasi-Static Acoustic Mapping of Helicopter Blade-Vortex Interaction Noise", Doctoral Dissertation, University of Maryland, 2004.

33. Farassat, F., "Derivation of Formulations 1 and 1A of Farassat", NASA TM-2007214853, March 2007.

34. Brentner, K.S. and Farassat,F., "Modeling aerodynamically generated sound of Helicopter Rotors”, Progress in Aerospace Sciences, 2003. vii.

35. Leishman, J.G., Principles of Helicopter Aerodynamics, Cambridge University Press, New York, 2006, Ch. 2-3.

36. Prouty, R.W., Helicopter Performance, Stability, and Control, PWS Engineering, Boston, MA, 1986, Ch. 3. 
37. Brooks, T.F., "Effect of Signal Jitter on the Spectrum of Rotor Impulsive Noise", Vertica, Vol. 12, No. 3. Pp 257-265, 1988.

38. http://www.bksv.com/doc/bp1851.pdf

39. http://www.bksv.com/doc/bp2212.pdf

40. http://www.bksv.com/doc/bp1650.pdf

41. Yin, J., van der Wall, B.G., and Oerlemans, S., "Acoustic Wind Tunnel Tests on Helicopter Tail Rotor Noise (HeliNOVI)", Journal of the American Helicopter Society, Vol. 53, No. 3, July 2008. 